\title{
Abstracts from the 2021 Atrial Fibrillation Symposium
}

E. Kevin Heist ${ }^{1}$, Moussa Mansour ${ }^{1}$, and Jeremy Ruskin ${ }^{1}$

${ }^{1}$ Massachusetts General Hospital

February 24, 2021

\begin{abstract}
Annual International Atrial Fibrillation Symposium January 29-31, 2021
\end{abstract}

Late-Breaking Clinical Trials and First Report Clinical Investigations: AFS 2021 -01 to -05

\section{AFS 2021-01}

Abstract Title: Radiofrequency Ablation for Paroxysmal or Persistent Atrial Fibrillation Using a LatticeTip Catheter: The Effect of Durable Lesions on One-Year Freedom from Atrial Arrhythmias

Author(s): Vivek Reddy, MD

Co-Author(s):

Petr Neuzil, MD PhD

Petr Peichl, MD PhD

Gediminas Rackauskas, MD PhD

Elad Anter, MD

Jan Petru, MD

Moritoshi Funasako, MD

Kentaro Minami, MD

Audrius Aidietis, MD PhD

Germanas Marinskis, MD PhD

Andrea Natale, MD

Hiroshi Nakagawa, MD PhD

Warren Jackman, MD

Josef Kautzner, MD PhD

Icahn School of Medicine at Mount Sinai

One Gustave L Levy Place Box 1030

Introduction | Objectives: There is scant data on the clinical efficacy of durable atrial ablation lesion sets, largely due to the challenges of achieving durability. But in a first-in-human trial (NCT04210622), a lattice-tip radiofrequency ablation catheter with a large thermal footprint was able to create point-by-point lesion sets that proved durable upon invasive remapping - including 99\% of pulmonary veins (PVs), and 
$91 \%, 100 \%$ \& $100 \%$ of mitral isthmus (MI), cavotricuspid (CTI) and left atrial roof lines, respectively. As all patients have now completed follow-up, herein we report the 1-year clinical outcomes.

Methods: In a 3-center single arm trial of paroxysmal (PAF) or persistent AF (PerAF), 8 operators performed PVI and, per operator preference, linear lesions. Other "atrial substrate" was not targeted. Using a custom electroanatomic mapping system, the saline-irrigated lattice catheter was used for temperaturecontrolled (Tmax $73^{\circ}-80^{\circ} \mathrm{C} ; 2-7 \mathrm{~s}$ ) point-by-point AF ablation. Follow up included 48-hr Holters at 3,6 and 12 mo; symptoms prompted additional monitoring. Compliance with monitoring was $77 \%$ overall $-91 \%$ for the 12 mo Holter.

Results: The cohort (65 pts; $40 \mathrm{PAF} / 25$ PerAF) received various ablation lesion sets: PVI (all pts), MI (5 PAF/17 PerAF), roof (8 PAF/16 PerAF), and CTI (22 PAF/20 PerAF). During remapping procedures $(\mathrm{n}=27 \mathrm{pts})$, additional ablation was performed in 8 pts. Most pts $(85 \%)$ ceased Class I/III AADs by last follow-up. After a mean follow up of $391 \pm 54$ days, sinus rhythm was maintained in $>90 \%$ of pts, across a range of sensitivity analyses (Figure ). There were no long-term safety events (e.g., PV stenosis, AE fistula). Limitations include: absence of continuous ECG monitoring, and limited number of pts/operators.

Conclusions: These data indicate that an anatomically-driven radiofrequency ablation lesion set, when durable, translates to excellent long-term clinical outcomes. It remains to be determined whether these outcomes are specific to the large thermal footprint lattice catheter, or is generalizable to other ablation catheters and energy sources.

AFS 2021-01

Uploaded File(s)

One-Year Outcomes of Lattice-Tip Ablation 


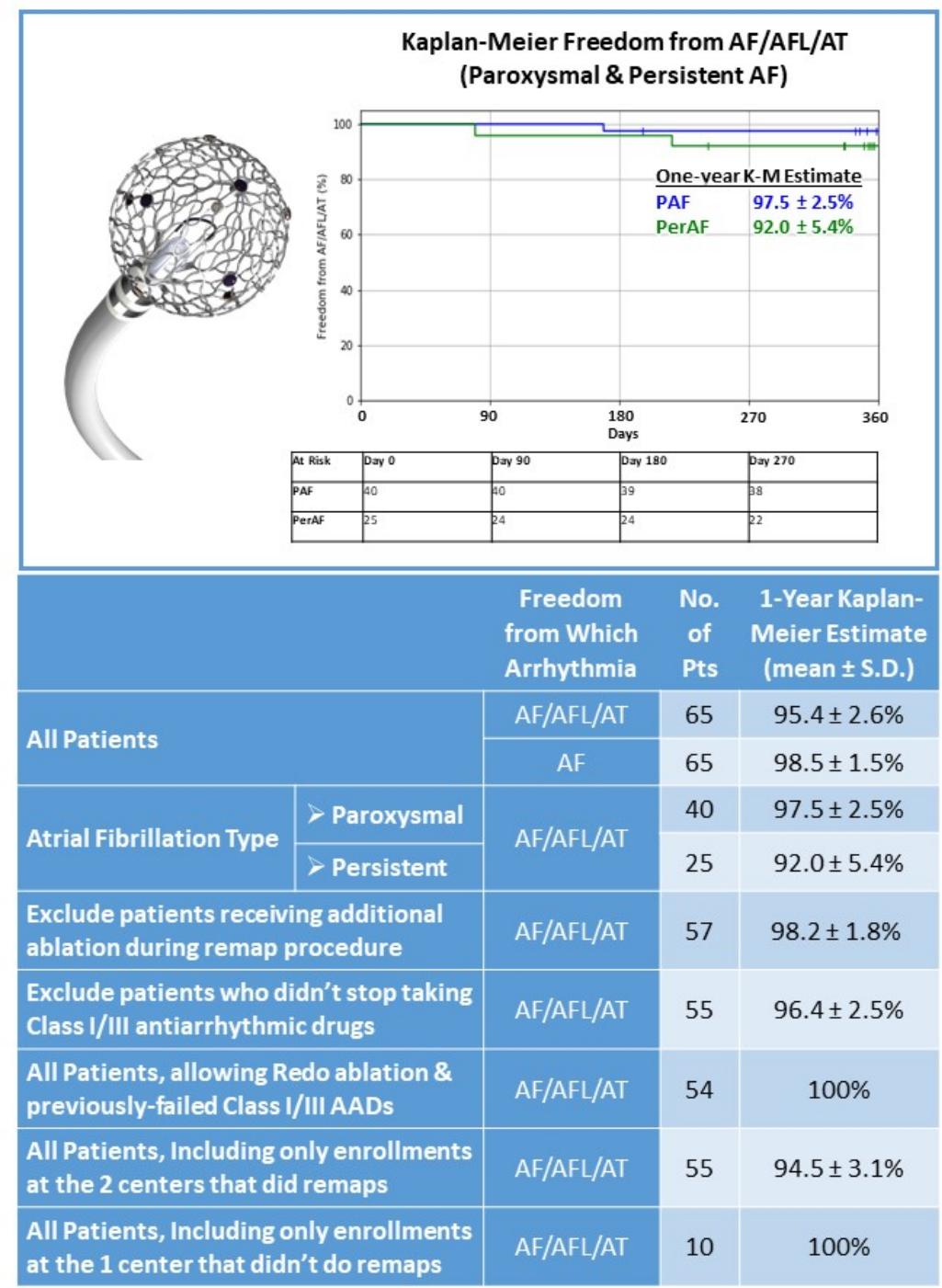

AFS 2021-02

Abstract Title: Lattice-Tip Focal Ablation of Persistent Atrial Fibrillation: Toggling Between Pulsed Field and Radiofrequency Energy

Author(s): Vivek Reddy, MD

Co-Author(s):

Petr Peichl, MD PhD

Gediminas Rackauskas, MD PhD

Elad Anter, MD

Jan Petru, MD 
Moritoshi Funasako", MD

Kentaro Minami, MD

Jacob Koruth, MD

Germanas Marinskis, MD PhD

Audrius Aidietis, MD PhD

Josef Kautzner, MD PhD

Petr Neuzil, MD PhD

Icahn School of Medicine at Mount Sinai

One Gustave L Levy Place Box 1030

Introduction | Objectives: The tissue selectivity of pulsed field ablation (PFA) provides safety advantages over RF ablation. One-shot PFA catheters for PVI exist, but they don't permit the flexibility in lesion sets, such as linear lesions, that are often desirable to treat patients with persistent atrial fibrillation (PerAF). In a first-in-human trial, we previously reported that a novel lattice-tip ablation catheter able to deliver either focal RF or PF energy, was used to treat AF with a strategy of either: i) PFA posteriorly and RFA anteriorly $(\mathrm{RF} / \mathrm{PF})$, or ii) $\mathrm{PFA}$ only $(\mathrm{PF} / \mathrm{PF})$. Herein, in a multicenter study, we present the performance characteristics with use of the lattice catheter to treat persistent AF.

Methods: The 8Fr lattice catheter has a compressible $9 \mathrm{~mm}$ nitinol tip, and is used with a custom mapping system and RF/PF generators (Sphere-9, Prism-1, HexaGen and HexaPulse, respectively; Affera Inc). In an IRB-approved clinical trial (NCT04141007, NCT04194307), toggling between energy sources, point-bypoint PV encirclement was performed using biphasic PFA (2-5 sec) posteriorly, and either temp-controlled irrigated RFA (Tmax $73^{\circ} \mathrm{C} ; 5 \mathrm{sec}$ ) or PFA anteriorly. PVI was confirmed with bidirectional pacing, and adenosine or after a 20 min wait. Linear lesions were placed with PFA or RFA.

Results: At 3 centers (6 operators), a 54-pt persistent AF cohort (age $62 \pm 9.6$ yrs; M / F = 44 / 10) underwent either RF/PF (20 pts; $56.9 \pm 18.5$ lesions/pt) or PF/PF (34 pts; $61.9 \pm 13.6$ lesions/pt) ablation. The PVI therapy duration time (transpired time from first to last lesion) was $23.6 \pm 8.8 \mathrm{~min} / \mathrm{pt}$. Linear lesions included 37 mitral (14 RF / $7 \mathrm{RF}+\mathrm{PF} / 16 \mathrm{PF}), 48 \mathrm{LA}$ roof (4 RF / $2 \mathrm{RF}+\mathrm{PF} / 42 \mathrm{PF}$ ) and $44 \mathrm{CTI}$ (32 RF / $12 \mathrm{PF}$ ) lines, with therapy duration times of $4.3 \pm 1.8,2.2 \pm 2.2$ and $2.2 \pm 1.6 \mathrm{~min} / \mathrm{pt}$, respectively. All lesion sets were acutely successful. Total fluoroscopy time was $4.3 \pm 2.5 \mathrm{~min}$. There were no device complications; there were 4 vascular injuries. Post-procedure EGD revealed no thermal injury in $18 \mathrm{RF} / \mathrm{PF}$ and $26 \mathrm{PF} / \mathrm{PF}$ pts.

Conclusions: To treat patients with persistent AF, the focal lattice catheter could safely and rapidly ablate AF using either a combined $\mathrm{RF} / \mathrm{PF}$ approach (capitalizing on the safety of PFA and the years of experience with RFA) or an entirely PF approach.

\section{AFS 2021-02}

\section{Uploaded File(s)}

Figure: Point-by-Point RF-PF Ablation of Persistent AF 


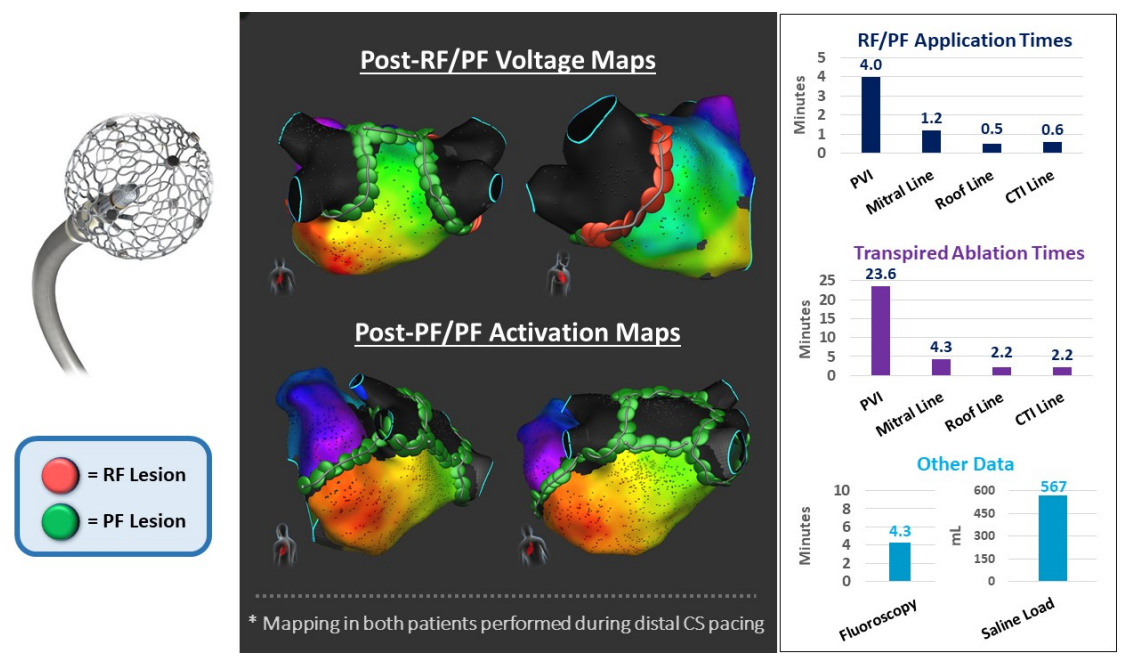

\section{AFS 2021-03}

Abstract Title: Hybrid epicardial-endocardial ablation vs. endocardial catheter ablation for longstanding persistent atrial fibrillation treatment: A sub-analysis from CONVERGE multi-center randomized controlled trial

Author(s): David DeLurgio, MD

Co-Author(s):

karl Crossen, MD

Jaswinder Gill, MD

Christopher Blauth, MD

Saumil Oza, MD

Anthony Magnano, MD

Mark Mostovych, MD

Michael Halkos, MD

Sreedhar Billakanty, MD

Steve Duff, MD

Christopher Stees, MD

Syed Ahsan, MD

John Yap, MD

Eric Espinal, MD

Otto Constantini, MD

David Gilligan, MD

Bruce Hook, MD

Tyler Taigen, MD

David Tschopp, MD 
Faraz Kerendi, MD

Christian Shults, MD

Manish Shah, MD

David Pederson, MD

Anil Rajendra, MD

Jose Osorio, MD

Jonathan Silver, MD

Hugh Calkins, MD

Emory St Joseph's

1364 Clifton Rd, Atlanta, GA

Introduction | Objectives: Long-standing persistent AF (LSPAF) is characterized by electroanatomical changes to left atrial substrate and clinical success with endocardial only ablation is limited. The CONVERGE multi-center randomized controlled trial demonstrated superior effectiveness of combined epicardialendocardial (hybrid Convergent, $\mathrm{HC}$ ) ablation to endocardial only catheter ablation (CA) for symptomatic, drug refractory, advanced AF treatment. A sub-analysis was conducted to compare the outcomes specifically in LSPAF.

Methods: In CONVERGE trial, a total of 153 adult symptomatic drug refractory patients were randomized to $\mathrm{HC}(\mathrm{N}=102 ; 38 \mathrm{LSPAF})$ or $\mathrm{CA}(\mathrm{N}=51 ; 27 \mathrm{LSPAF})$. Primary effectiveness was freedom from atrial arrhythmia through 12 months absent new/increased dose of previously failed anti-arrhythmic drugs (AADs). Secondary endpoints included freedom from AF, AF burden reduction, 18-month effectiveness (by 7-day Holter), and quality-of-life (QoL). Primary safety was major adverse event rate through 30-days post procedure.

Results: Baseline demographics in the LSPAF subgroup were similar in HC and CA arms - mean BMI (33 Vs 35), mean LA size (4.5 Vs $4.3 \mathrm{~cm})$, cardioversion within 12 months (53\% Vs 59\%), mean AF duration (6.0 Vs 5.9 years). Mean ablation time in $\mathrm{HC}$ was 217 minutes (endocardial $=140 \mathrm{~min}$, epicardial $=77 \mathrm{~min}$ ) and 181 minutes in CA. As a result of epicardial ablation, on an average 41 minutes of less endocardial ablation was required in $\mathrm{HC}$ arm. Freedom from atrial arrhythmia was significantly higher in $\mathrm{HC}$ compared to the CA. The QoL and AF symptoms, as assessed by SF-36 and AFSS questionnaires at baseline and 12 months post-ablation, significantly improved in $\mathrm{HC}$ arm. No deaths or fatal events were reported in either arm. Three patients $(7.8 \%)$ in $\mathrm{HC}$ and none in CA had primary safety events.

Conclusions: LSPAF subgroup analysis from the CONVERGE trial showed significant improvements in freedom from atrial arrhythmias and AF burden reduction with hybrid Convergent ablation compared to endocardial ablation only, with quality-of-life benefits. These are important findings as LSPAF patients currently have limited effective treatment options.

\section{AFS 2021-03 Uploaded File(s)}

Outcomes of Hybrid Convergent Vs Endocardial Ablation for Treatment of Long-standing Persistent AF 


\begin{tabular}{|c|c|c|c|}
\hline Parameter & $\begin{array}{l}\text { Hybrid Convergent (HC, } \\
\mathrm{N}=38 \text { ) }\end{array}$ & $\begin{array}{l}\text { Endocardial ablation } \\
(\mathrm{CA}, \mathrm{N}=27)\end{array}$ & Statistical significance \\
\hline \multicolumn{4}{|c|}{ Freedom from AF/AFL/AT absent new AAD/increased dose previously failed AAD } \\
\hline 12 months & $65.8 \%$ & $37.0 \%$ & $P=0.022$ \\
\hline 18 months & $60.5 \%$ & $25.9 \%$ & $P=0.006$ \\
\hline \multicolumn{4}{|c|}{ Freedom from AF/AFL/AT absent AADs } \\
\hline 12 months & $52.6 \%$ & $25.9 \%$ & $P=0.031$ \\
\hline 18 months & $47.4 \%$ & $22.2 \%$ & $P=0.038$ \\
\hline \multicolumn{4}{|c|}{ Freedom from AF/AFL/AT regardless of AADs } \\
\hline 12 months & $73.7 \%$ & $44.4 \%$ & $P=0.017$ \\
\hline 18 months & $68.4 \%$ & $33.3 \%$ & $P=0.005$ \\
\hline \multicolumn{4}{|c|}{ Freedom from AF absent new AAD/increased dose previously failed AAD } \\
\hline 12 months & $71.1 \%$ & $37 \%$ & $P=0.006$ \\
\hline 18 months & $68.4 \%$ & $29.6 \%$ & $\mathrm{P}=0.002$ \\
\hline \multicolumn{4}{|c|}{$\geq 90 \%$ AF Burden Reduction absent new AAD/increased dose previously failed AAD } \\
\hline 12 months & $78.9 \%$ & $46.2 \%$ & $\mathrm{P}=0.007$ \\
\hline 18 months & $73 \%$ & $36 \%$ & $\mathrm{P}=0.004$ \\
\hline \multicolumn{4}{|c|}{ Freedom from cardioversion } \\
\hline $\begin{array}{l}\text { From procedure through } \\
12 \text { months }\end{array}$ & $71 \%$ & $41 \%$ & $P=0.015$ \\
\hline \multicolumn{4}{|l|}{ Mean ablation time } \\
\hline Epicardial ablation time & $77 \mathrm{~min}$ & Not applicable & \\
\hline Endocardial ablation time & $140 \mathrm{~min}$ & $181 \mathrm{~min}$ & $\begin{array}{l}\mathrm{P}=0.006 \\
41 \text { min saving in } \\
\text { endocardial ablation time } \\
\text { as a result of epicardial } \\
\text { ablation. }\end{array}$ \\
\hline
\end{tabular}

\section{AFS 2021-04}

Abstract Title: Hybrid thoracoscopic surgical and transvenous catheter ablation versus transvenous catheter ablation in persistent and longstanding persistent atrial fibrillation: 1-year results of the HARTCAPAF randomized controlled trial

Author(s): Bart Maesen, MD, PhD

\section{Co-Author(s):}

Vanessa Weberndörfer, MD - Fellow cardiac electrophysiology, PhD candidate, Maastricht University Medical Centre

Mindy Vroomen, MD, PhD - Maastricht University Medical Centre

Justin G. Luermans, MD, PhD - Cardiac Electrophysiologist, Maastricht University Medical Centre

Elham Bidar, MD, PhD - Cardiothoracic Surgeon, Maastricht University Medical Centre

Sevasti-Maria Chaldoupi, MD, PhD - Cardiac Electrophysiologist, Maastricht University Medical Centre

Claudia A.J. van der Heijden, MD - Resident, PhD-Candidate, Maastricht University Medical Centre

Kevin Vernooy, MD, PhD - Cardiac Electrophysiologist, Maastricht University Medical Centre

Brigitte Essers, MsC, PhD - Statistician, Maastricht University Medical Centre 
Sander MJ van Kuijk, MsC, PhD - Statistician, Maastricht University Medical Centre

Jos G. Maessen, MD, PhD - Head of Cardiothoracic Surgery, Maastricht University Medical Centre

Harry J.G.M. Crijns, MD, PhD - Head of Cardiology, Maastricht University Medical Centre

Laurent Pison, MD, PhD - Cardiac Electrophysiologist, Ziekenhuis Oost Limburg

Mark La Meir, MD, PhD - Head of Cardiac Surgery, UZ Brussels

Bart Maesen, MD, PhD - Cardiothoracic Surgeon, Maastricht University Medical Centre

Maastricht University Medical Centre

Maastricht University Medical Centre Departement of Cardiothoracic Surgery P. Debyelaan 25, 6229 HX Maastricht, Netherlands

Introduction | Objectives: Success rates of conventional transvenous endocardial ablation of nonparoxysmal atrial fibrillation (AF) vary considerably and long-term results are disappointing. Meanwhile, reported results after hybrid ablation, combining thoracoscopic epicardial surgical and endocardial catheter ablation, are more encouraging. Here, we present the results of the HARTCAP-AF, first randomized controlled trial comparing both approaches to test the hypothesis that hybrid ablation is more effective than conventional catheter ablation in patients with non-paroxysmal AF.

Methods: Forty-one patients with non-paroxysmal AF were prospectively randomized to either one-stage hybrid ablation (HA) or conventional catheter ablation (CA). In both groups, pulmonary vein (PV) isolation and a box-lesion consisting of an inferior and superior connecting lesion were created.In HA, endocardial validation of block, and touch-up ablation, if needed, was performed. During follow-up, 24h Holter-monitoring was performed at 3 and 6 months, 7-days Holter-monitoring at 12 months. The primary effectiveness endpoint was freedom from any atrial arrhythmia $>5 \mathrm{~min}$ without the use of class I/III AADs 12-months after ablation. Quality of life (QOL) at 1 year was evaluated using the EQ-5D-5L questionnaire.

Results: At 12 months, freedom from any atrial arrhythmia off AADs was 83\% (15/18) after HA and 45\% after CA $(10 / 22)(\mathrm{p}=0.015)$. One patient could not complete the $12 \mathrm{~m}$ follow-up. There were 3 complications: 1 pericardial tamponade and 1 femoral AV-fistula in HA-group, 1 femoral artery bleeding needing surgical repair in CA-group. There were no deaths or strokes. At 12-months, QOL did not differ significantly between HA and CA $(\mathrm{p}=0.95)$. There were less cardiovascular rehospitalizations in HA.

Conclusions: This is the first randomized controlled trial comparing hybrid with conventional ablation in non-paroxysmal AF patients. After 1 year, hybrid ablation resulted in a significantly higher freedom of atrial arrhythmias without increasing the number of associated major adverse events.

\section{AFS2021-04}

Uploaded File(s)

Arrhythmia-free Survival after Hybrid and Catheter Ablation at 12 months without the use of AAD 


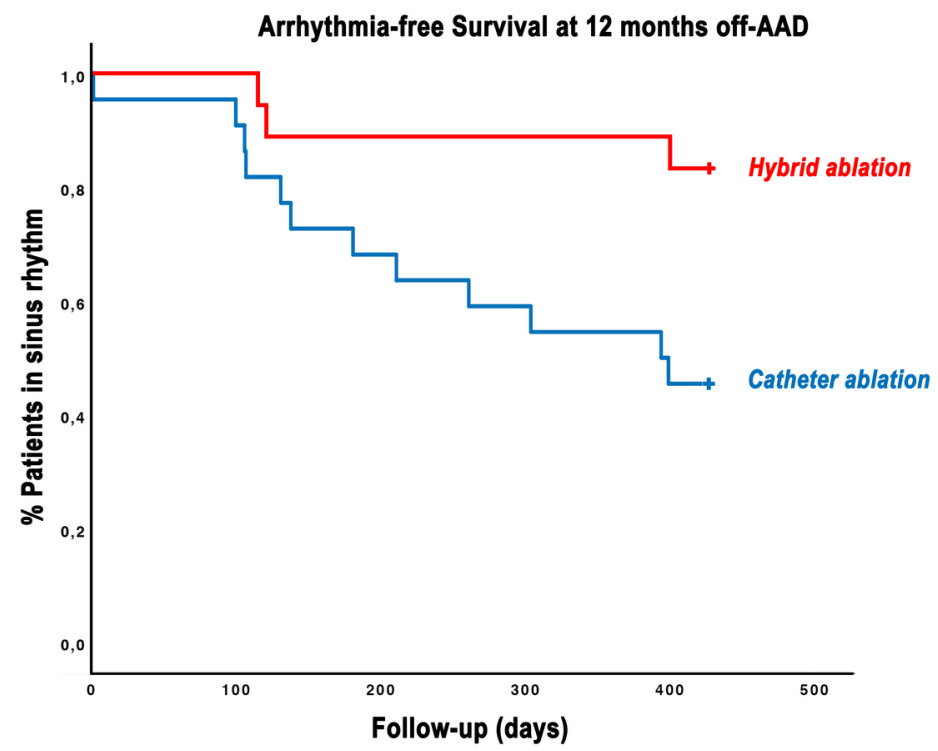

AFS 2021-05

Abstract Title: Efficacy and Safety of Esophageal Deviation in Atrial Fibrillation Ablation Using an Inflatable Balloon: A Prospective Single-Arm Study

Author(s): Weeranun D. Bode, MD

Co-Author(s):

Michael Andrawes, MD

Agnieszka Lesicka, MD

Grace Ha, MS

Kevin Heist, MD PhD

Dinxing Qin, MD

Kathryn Slattery, CRNA

Christopher Houghtaling, BS MA

Nathan Van Houzen, MD

Molly Campbell, DNP CRNA

Abraham Sonny, MD

Alexander Kuo, MD MS

Kenneth Shelton, MD

Jeremy Ruskin, MD

Adam Dalia, MD MBA

Michael Fitzsimons, MD

Moussa Mansour, MD 


\section{Massachusetts General Hospital}

Cardiac Arrhythmia Service, Massachusetts General Hospital Bigelow Building 855 Fruit Street, Boston, MA, 02114

Introduction | Objectives: The DV8 probe (Minneapolis, Minnesota) consists of a shaped balloon that is inserted into the esophagus and inflated, which results in its deviation away from the left atrium. Prior studies have demonstrated that moving the esophagus by $1 \mathrm{~cm}$ away from the ablation location would result in significant reduction of esophageal heating. This study sought to evaluate the efficacy and safety of the DV8 tool in moving the esophagus.

Methods: This is a single center, open-label, single-arm study enrolling patients undergoing radiofrequency (RF) ablation for paroxysmal and persistent atrial fibrillation ablation (AF). The DV8 device was inserted at the beginning of the procedure. During pulmonary vein (PV) and posterior wall ablation, the DV8 balloon was inflated and deviated the esophagus to the contralateral side. The primary endpoint was the success rate of moving the closest edge of the esophagus by a minimum of $10 \mathrm{~mm}$ from the ablation line of the ipsilateral $\mathrm{PV}$ pairs. The secondary endpoints were the success rate of moving the esophagus by a minimum of 20 $\mathrm{mm}$, the incidence of esophageal laceration as assessed by Barium swallow, incidence of PV reconnection 30 minutes after ablation, and procedure time.

\section{Results:}

A total of 43 patients underwent DV8 device placement. The primary endpoint was reached in 42 patients (success rate $97.7 \%$ ) with a mean deviation distance of $15.3 \pm 4.3 \mathrm{~cm}$. Nine patients achieved a mean deviation distance of [?] $20 \mathrm{~mm}$. There was no esophageal injury found during the Barium swallowing tests. The incidence of PV reconnection with adenosine, isoproterenol infusion, and waiting 30 minutes after ablation was 23\%. Mean fluoroscopy, total procedure, and ablation times were $23+-6,211+-32$, and 53 +- 15 minutes, respectively. The median follow-up time was 369 days. AF recurred in 9 patients during the follow-up period (freedom from AF recurrence of $72.3 \%$ ). None of the patients had any long-term complications that were related to DV8 device placement.

Conclusions: In this single-center prospective study, we report that use of the DV8 device for esophageal deviation during AF ablation is effective and safe.

General Abstracts: AFS 2021 -06 to -56

\section{AFS 2021-06}

Abstract Title: Comparison of XY versus XY/XZ Mapping During Pulmonary Vein Isolation Using the HD Grid

Author(s): Sujay Sreenivasan, A.B. Music

\section{Co-Author(s):}

Michael Ahmed, B.S. Cellular Biology

Alexander Sweeting, B.S. Biology

Jacob Lawing, B.S.

James Frix, B.S.

Kent Nilsson, M.D.

AU/UGA Medical Partnership

University of Georgia Health Sciences Campus Prince Avenue Athens, GA 30602

Introduction | Objectives: Pulmonary vein isolation (PVI) has emerged as a cornerstone of management for atrial fibrillation. Traditional mapping using a circular catheter collects electrograms (EGMs) along the 
circumference of the vein (XY). Cardiac fibers, however, enter the veins at oblique angles (XY and XZ). For fibers traveling longitudinally into the vein (XZ), perpendicular to the circumference of the vein, EGMs may not be detected. Multidimensional mapping using the Advisor HD Grid catheter, however, overcomes this shortcoming by mapping in both the XY and XZ dimensions.

Methods: All patients who underwent PVI at Piedmont Athens Regional over a six-month window, January - June 2017 (circular) and seventeen-month window, January 2019 - May 2020 (HD Grid)) were included in the study. A retrospective chart review was performed to determine recurrence rates between the two cohorts.

Results: 278 patients with paroxysmal atrial fibrillation met inclusion criteria, with 84 patients undergoing ablation with a Spiral Catheter, and 194 patients undergoing ablation with the Advisor HD Grid Catheter. Of patients with paroxysmal atrial fibrillation, $22.1 \%$ of patients had a reoccurrence of atrial fibrillation at 12 months when mapped with the spiral catheter versus $16.0 \%$ with HD grid $(p=0.104)$.

Conclusions: There was no significant difference in reoccurrence rate of atrial fibrillation when using either the Advisor HD Grid or Spiral Catheter for patients with paroxysmal atrial fibrillation.

AFS 2021-06

\section{Uploaded File(s)}

\section{Paroxysmal Atrial Fibrillation - Reoccurrence Rate}

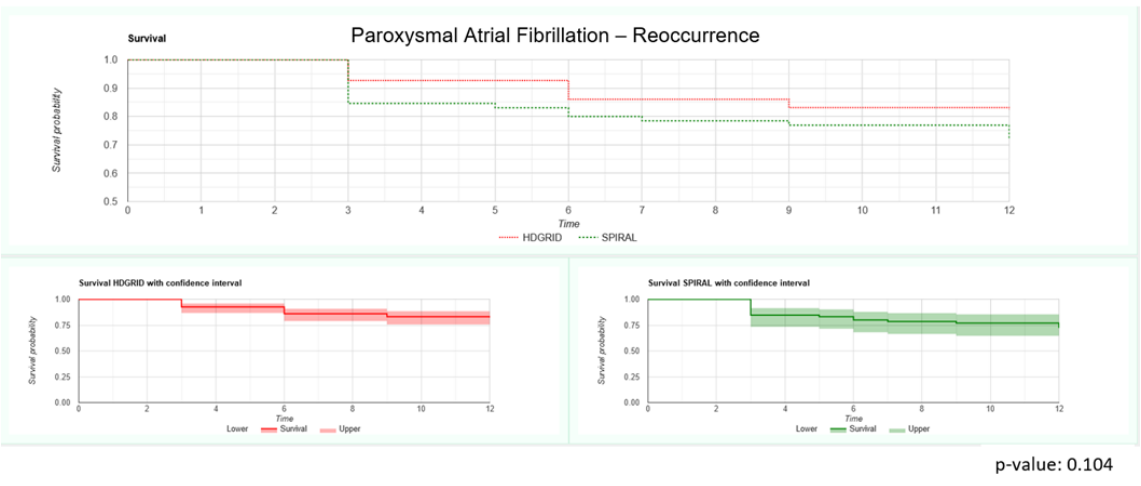

\section{AFS 2021-07}

Abstract Title: Atrial Fibrillation in Patients Hospitalized with COVID-19: Incidence, Predictors, Outcomes and Comparison to Patients with Influenza."

Author(s): Daniel Musikantow, MD

Co-Author(s):

Mohit Turagam, MD

Samantha Sartori, PhD

Edward Chu, MD

Iwanari Kawamura, MD

Poojita Shivamurthy, MD

Mahmoud Bokhari, MD

Connor oates, MD 
Chi Zhang, MD

Christopher Pumill, MD

Waqas Malick, MD

Helen Hashemi, MD

Tania Ruiz-maya, MD

Michael Hadley, MD

Jonathan Gandhi, MD

Dylan Sperling, MD

William Whang, MD

Jacob Koruth, MD

Marie-noelle Langan, MD

Aamir Sofi, MD

Anthony Gomes, MD

Stephanie harcum, MS

Sam Cammack, MS

Betsy Ellsworth, MSN

Srinivas Dukkipati, MD

Martin Goldman, MD

Jonathan Halperin, MD

Valentin Fuster, MD, PhD

Vivek Reddy, MD

Icahn School of Medicine at Mount Sinai

One Gustav Levy Place

Introduction | Objectives: Coronavirus Disease 2019 (COVID-19) results in increased levels of inflammatory markers previously associated with atrial arrhythmias. However, little is known about the incidence or specificity of these arrhythmias in COVID-19 or their association with outcomes. The purpose of this paper is to determine the incidence, predictors and outcomes of atrial fibrillation and flutter (AF/AFL) in patients hospitalized with COVID-19 compared to those hospitalized with the Influenza virus.

Methods: This is a retrospective analysis involving consecutive patients from five hospitals during the height of the COVID-19 pandemic in New York City. The primary analysis involved 3,970 patients admitted with PCR-positive COVID-19 between 2/4/2020-4/22/2020 with manual review performed of 1,110 these patients. The comparator arm included 1,420 patients with influenza hospitalized between $1 / 1 / 2017-1 / 1 / 2020$ such that no temporal overlap occurred between groups.

Results: Among 3970 inpatients with COVID-19, the incidence of AF/AFL was $10 \%(\mathrm{~N}=375)$ and in patients without a history of atrial arrhythmias, $4 \%(\mathrm{~N}=146)$. Patients with new-onset AF/AFL were older and had higher levels of inflammatory markers including C-reactive protein $(232 \mathrm{vs} 175 \mathrm{mg} / \mathrm{L}, \mathrm{P}<0.0001)$ and Interleukin-6 (93 vs $68 \mathrm{pg} / \mathrm{ml}, \mathrm{P}=0.001$ ), and more myocardial injury (Troponin-I: $0.2 \mathrm{vs} 0.06 \mathrm{ng} / \mathrm{ml}$, $\mathrm{P}<0.0001) . \quad \mathrm{AF} / \mathrm{AFL}$ were associated with increased mortality $(46 \%$ vs $26 \%, \mathrm{P}<0.0001)$ and ischemic stroke $(1.6 \%$ vs $0.6 \%, \mathrm{P}=0.05)$. Manual review captured a somewhat higher prevalence of $\mathrm{AF} / \mathrm{AFL}(13 \%$, 
$\mathrm{N}=140$ ) compared to automated abstraction. Compared to inpatients with COVID-19, patients with Influenza $(\mathrm{N}=1420)$ had similar rates of $\mathrm{AF} / \mathrm{AFL}(12 \%, \mathrm{n}=163)$ but lower overall mortality. The presence of AF/AFL during hospitalization correlated with similarly increased mortality in both COVID-19 (RR 1.77) and Influenza (RR 1.78).

Conclusions: AF/AFL occurred in a subset of patients hospitalized with COVID-19, and was associated with elevated markers of inflammation and disease severity as well as adverse outcomes. The incidence and associated increase in mortality of AF/AFL in COVID-19 was similar to that observed with Influenza.

AFS 2021-07

Uploaded File(s)

Survival Stratified by AF/AFL in COVID-19 versus Influenza

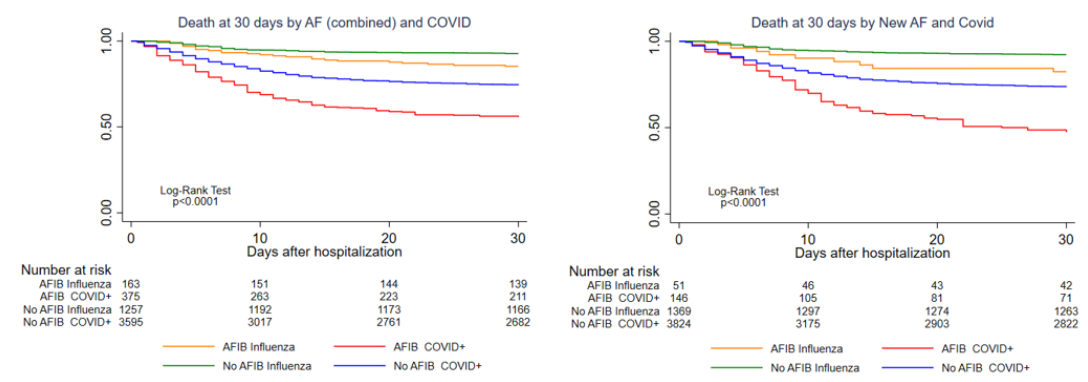

\section{AFS 2021-08}

Abstract Title: High Density Mapping Compared to Low Density Mapping in Ablation of Atypical Atrial Flutter

Author(s): Jippe Balt, MD PhD

Co-Author(s):

Martijn Klaver, MD - Cardiologist, St Antonius Hospital

Bob Abeln, MD - Cardiologist, St Antonius Hospital

Bakhtawar Mahmoodi, MD PhD - St Antonius Hospital

Vincent Van Dijk, MD PhD - Electrophysiologist, St Antonius Hospital

Maurits Wijffels, MD PhD - Electrophysiologist, St Antonius Hospital

Lucas Boersma, Professor - Electrophysiologist, St Antonius Hospital

St Antonius Hospital

Koekoekslaan 13425 CM Nieuwegein The Netherlands

Introduction | Objectives: High-density (HD) mapping of ablation targets may increase success. The purpose of the present study was to compare HD mapping with conventional mapping in ablation of AAFL.

Methods: We compared baseline and procedural characteristics, procedural success, safety and outcome of mapping and ablation of atypical flutter in three groups. (1) HD Grid catheter + the high-density electro anatomical mapping (EAM) system EnSite Precision (2) standard 10-pole circular mapping catheter (CMC) + Ensite Precision (3) CMC + the low density Ensite Velocity EAM. 
Results: 82 patients were included. Mapping of 142 atypical atrial flutters was attempted. Acute ablation success was $78 \%, 68 \%$ and $51 \%$ in groups 1,2 and $3(\mathrm{p}=0.037$ between group 1 and 3 , NS between groups otherwise). 8, 27 and $36 \%$ of flutters were unmappable in groups 1, 2, and 3, respectively ( $\mathrm{p}<0.05$ between group 1 and both groups 2 and 3, respectively). AAFL recurrence at 1-year FU was 26\%,36\% and 62\% in groups 1,2 and 3 ( $\mathrm{p}=0.007$ between group 1 and $3, \mathrm{p}=0.05$ between group 2 and 3 ). AAFL-free survival was significantly higher in patients mapped with Precision than with Velocity $(\mathrm{p}=0.011)$. No serious adverse events occurred within 30 days.

Conclusions: Acute procedural success and freedom from recurrent AAFL at 1-year are significantly higher using the HD-Grid mapping catheter in combination with the high-density EnSite Precision system, as compared to a decapolar circular mapping catheter and the low-density EnSite Velocity EAM system. HD Mapping is safe.

\section{AFS 2021-09}

Abstract Title: Voltage Mapping and Pulmonary Vein Isolation in Master Athletes with Atrial Fibrillation Author(s): Eric Pagan, MD

\section{Co-Author(s):}

Nicholas Beccarino, MD

David Chang, MD

Eric Dulmovits, BS

Stuart Beldner, MD

Northwell Health

300 Community Drive, Manhasset, NY 11030

Introduction | Objectives: Master athletes encompass a wide range of exercise enthusiasts. At the extreme there is an increased risk of atrial fibrillation (AF). Therapies aimed at rate or rhythm control are often limited given unfavorable side effects. Although studies suggest an increase in left atrial (LA) fibrosis in this population, minimal electrophysiologic data exist regarding the LA voltage mapping and the efficacy of AF ablation with pulmonary vein isolation (PVI).

Methods: In a retrospective single-center study, we reviewed AF ablations (pulmonary vein isolation and assessment/ablation of non-pulmonary vein triggers) performed in "extreme" master athletes with AF. We define these patients as those who have repeatedly competed in long distance endurance events for a $>10$ year period. Bipolar voltage mappings were reviewed using CARTO (Biosense Webster, Diamond Bar, USA) and LA scarring was defined as an area of less than $0.1 \mathrm{mV}$. All patients were monitored as outpatient for AF recurrence.

Results: Between January 2018 and January 2020, 14 patients (nine marathon runners, four long distance cyclers, and one marathon swimmer) underwent AF ablations. The patient demographics are shown in Table 1. All patients in the cohort were male with an average $\mathrm{CHA}_{2} \mathrm{DS}_{2}$ VASc score of $1.2 \pm 0.9$ and left atrial volume of $34.4 \mathrm{cc} / \mathrm{m}^{2} \pm 9.9$. A total of seven patients (50\%) had persistent AF. One patient (7.1\%) had LA scar on the bipolar voltage mapping, whom also had a non-pulmonary vein trigger of AF. Bidirectional blocks of the four pulmonary veins were achieved by radiofrequency (RF) ablation in all patients. Freedom from documented recurrence of AF up to 24 months was $92.9 \%$. One patient (7.1\%) had recurrence of AF at 14 months and underwent successful cardioversion.

Conclusions: In our series of extreme master athletes with AF, the incidence of LA scarring on the bipolar voltage mapping was low and the recurrence of AF following PVI by RF ablation was minimal.

\section{AFS 2021-09 Uploaded File(s)}


Table 1: Patient Demographics

\begin{tabular}{|l|c|}
\hline Characteristic & $\mathbf{N}=\mathbf{1 4}$ \\
\hline Male Sex & $14(100 \%)$ \\
\hline Age & $64.1 \pm 5.6$ \\
\hline Body mass index $\left(\mathrm{kg} / \mathrm{m}^{2}\right)$ & $26.9 \pm 4.4$ \\
\hline Left Atrial Volume $\left(\mathrm{cc} / \mathrm{m}^{2}\right)$ & $34.4 \pm 9.9$ \\
\hline Paroxysmal Atrial Fibrillation & $7(50 \%)$ \\
\hline Persistent Atrial Fibrillation & $7(50 \%)$ \\
\hline CHA2DS2-VASc score & $1.2 \pm 0.9$ \\
\hline Hypertension & $6(42.9 \%)$ \\
\hline Diabetes mellitus & 0 \\
\hline History of Cerebral Vascular Accident & 0 \\
\hline Vascular Disease & $2(14.2 \%)$ \\
\hline Congestive Heart Failure & $1(7.1 \%)$ \\
\hline Tobacco use & $1(7.1 \%)$ \\
\hline Alcohol use & 0 \\
\hline Obstructive Sleep Apnea & $1(7.1 \%)$ \\
\hline Chronic Obstructive Pulmonary Disease & $1 \%)$ \\
\hline
\end{tabular}

AFS 2021-10

Abstract Title: Safety and Efficacy Analysis of Warfarin versus Direct Oral Anticoagulants use in Atrial Fibrillation

Author(s): Meagan Goodier, MSN, FNP (c)

Co-Author(s):

Julianne Murthi, MSN, FNP

MCPHS

19 Foster St, Worcester, MA 01608

Introduction | Objectives: Purpose: The aim of this project is to determine if warfarin or direct oral anticoagulants are more effective at preventing adverse outcomes in those who have a diagnosis of atrial fibrillation. Background:Atrial Fibrillation is a disease that effects millions of individuals with high levels of morbidity and mortality due to its complications. Oral anticoagulants are used to help prevent adverse effects in the safest and most effective way possible. Objective: To determine which oral anticoagulant is the safest and effective to use in those with atrial fibrillation.

Methods: Methods: A systematic review of published studies was completed to determine which oral anticoagulant would be the safest and most effective anticoagulant for those with a diagnosis of atrial fibrillation.

Results: Results: The use of apixaban decreases the risk of stroke and other systemic embolisms in those with AFib, while also offering a decreased risk of bleeding events due to use of an OAC. 
Conclusions: Implications for practice: The project has the potential to provide APRNs guidance on understanding which oral anticoagulant is best suited for their patient. APRNs and other prescribers will be able to identify risks associated with prescribing treatment for their patients in order to prevent adverse events. Limitations: Although well published articles were used to determine the results, this research project was not able to use primary data to complete the research and depending on secondary data.

AFS 2021-10

\section{Uploaded File(s)}

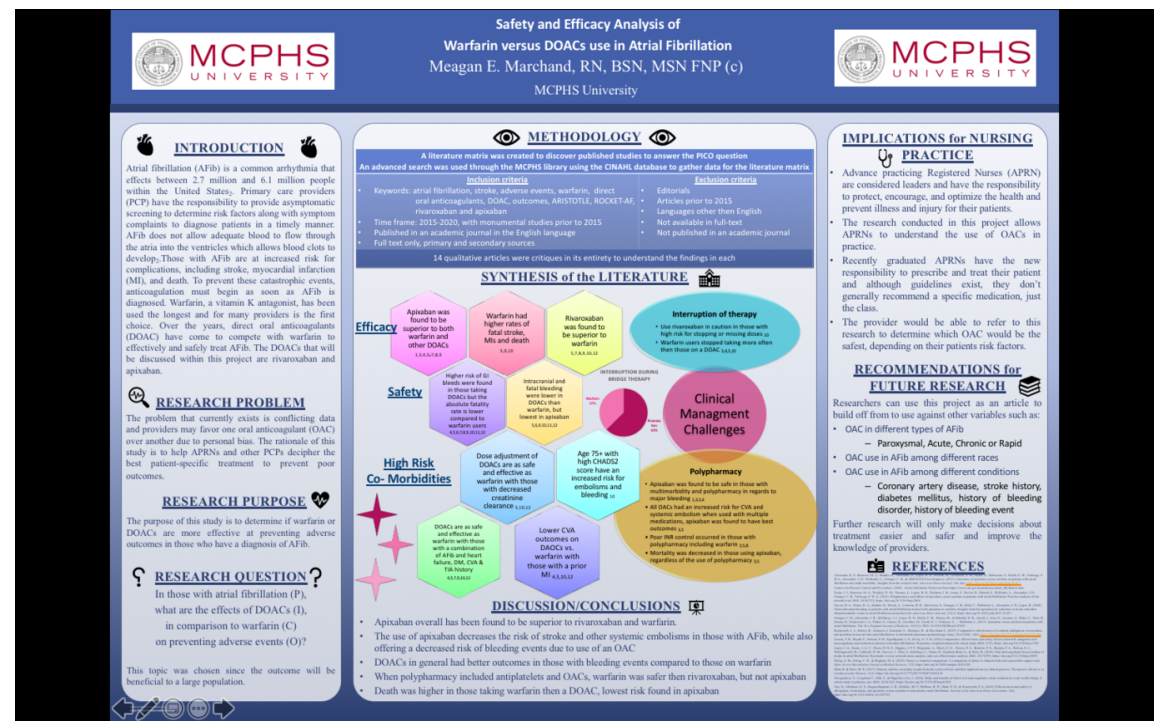

\section{AFS 2021-11}

Abstract Title: Features of metabolic disorders in very elderly patients with atrial fibrillation

Author(s): Svetlana B Topolyanskaya, PhD

\section{Co-Author(s):}

Leonid Dvoretski, MD - professor, First Moscow State Medical Universilty (Sechenov University)

Tatyana Eliseeva, No - physician, War Veterans Hospital N3

Olga Vakulenko, No - Head, War Veterans Hospital N3

First Moscow State Medical Universilty (Sechenov University)

\section{8-2, Trubetskaya str., Moscow}

Introduction | Objectives: There are few data available on metabolic disorders in very elderly patients with atrial fibrillation, therefore we investigate these disorders.

Methods: Cross sectional data from 475 hospitalized patients with coronary artery disease were analyzed. The patients' age ranged from 75 to 106 years; the mean age was $88.3+5.1$ years. The majority of study participants $(74.1 \%)$ were women.

Results: Prevalence of atrial fibrillation in this study was 34.3\%. Atrial fibrillation was diagnosed more often in patients with hyperuricemia - in $43 \%$ versus $29.4 \%$ of patients with normal uric acid $(\mathrm{p}=0.001)$. Hyperuricemia was significantly associated with risk of atrial fibrillation (hazard ratio=1.8 (95\% CI=1.22.7); $\mathrm{p}=0.001$ ). Left atrium dilatation was registered in $82.6 \%$ of patients with hyperuricemia whereas in 
patients with normal uric acid level - in $77.1 \%$ of cases $(\mathrm{p}=0.004)$. Concentrations of all blood lipids in patients with atrial fibrillation were significantly lower than in patients without this arrhythmia. The largest differences were in total cholesterol ( $4.4 \mathrm{vs} 5.1 \mathrm{mmol} / \mathrm{l}$ in patients without atrial fibrillation; $\mathrm{p}<0.0001$ ), in triglycerides $(1.2$ and $1.6 \mathrm{mmol} / \mathrm{L} ; \mathrm{p}=0.00002)$ and in LDL cholesterol level $(2.4$ and $3.0 \mathrm{mmol} / \mathrm{L} ; \mathrm{p}=0.0007)$. Highly significant inverse correlations were established between left atrium diameter and total cholesterol $(\mathrm{r}=-0.25 ; \mathrm{p}<0.0001)$, LDL cholesterol $(\mathrm{r}=-0,22 ; \mathrm{p}=0.01)$ and HDL cholesterol $(\mathrm{r}=-0.25 ; \mathrm{p}=0.003)$ levels. $50 \%$ of obese patients were diagnosed with atrial fibrillation, while in the non-obese group this arrhythmia was registered in $31.3 \%(\mathrm{p}=0.001)$. Obese patients had larger left atrium diameter compared to patients with normal body mass index ( 45.2 vs $43.5 \mathrm{~mm}$, respectively; $\mathrm{p}=0.007$ ). In patients with atrial fibrillation and without this arrhythmia, the mean fasting blood glucose concentrations did not differ $-6.0+2.1$ and $6.2+$ $2.4 \mathrm{mmol} / \mathrm{L}$, respectively $(\mathrm{p}=0.64)$. Atrial fibrillation was registered in $34.8 \%$ of patients with diabetes mellitus and $34.5 \%$ of patients without diabetes $(\mathrm{p}=0.95)$.

Conclusions: The study results indicate some features of different metabolic disorders in very elderly patients and centenarians with atrial fibrillation.

\section{AFS 2021-12}

Abstract Title: Assessing the Relationship of Applied Force and Ablation Duration on Lesion Size Using a Diamond Tip Catheter Ablation System

Author(s): Atul Verma, MD

Co-Author(s):

Megan Schmidt, PhD

Jean-pierre Lalonde, BS

David Ramirez, BS

Michael Getman, BS, MS

Southlake Regional Health Centre

596 Davis Dr. Newmarket, Ontario L3Y 2P9

Introduction | Objectives:Background: Contact force has proven to be influential for lesion formation in power-controlled radiofrequency (RF) ablation. However, this technology provides a surrogate for lesion formation. A diamond tip catheter ablation system was specifically designed to facilitate accurate temperature control and low irrigation for higher power and shorter duration radiofrequency delivery. Objectives: Lesion formation and morphology from a temperature-controlled diamond tip RF ablation catheter is not well described. We hypothesize that lesion formation from a temperature-controlled RF system is independent of applied force over short application duration.

Methods:Methods: This study examined lesion depth, lesion width, temperature, and ablation parameters of the DiamondTemp Ablation (DTA, Medtronic, Inc.) system for ablation applications delivered with a 5 second application duration and applied force $(5,10$, and $30 \mathrm{~g})$. Lesions from perpendicular RF applications were analyzed in a stepwise fashion from a computational model, thermochromic gel data $(n=12)$, and porcine thigh preparation $(\mathrm{n}=76)$ experiments.

Results:Results: Varying applied force $(5,10$, and 30g) across an application duration of 5 seconds consistently generated comparable lesion dimensions for each model. In the computational model, lesion depths from a $5 \mathrm{sec}$ application with 5,10 , and $30 \mathrm{~g}$ of applied force were similar $(3.7,3.9$, and $3.7 \mathrm{~mm}$, respectively). Also, the $5 \mathrm{sec}$ lesion depths in the gel model were consistent across applied force $(5 \mathrm{~g}=$ $3.2 \pm 0.12 \mathrm{~mm}, 10 \mathrm{~g}=3.5 \pm 0.13 \mathrm{~mm}, 30 \mathrm{~g}=3.5 \pm 0.20 \mathrm{~mm})$. In the thigh model, the 5,10 , and $30 \mathrm{~g}$ applied forces for $5 \mathrm{sec}$ created lesion depths of $3.1 \pm 0.46 \mathrm{~mm}, 3.2 \pm 0.97 \mathrm{~mm}$, and $3.2 \pm 1.05 \mathrm{~mm}$, respectively. 
Conclusions: Conclusion: Lesion dimensions with the DTA temperature-controlled RF ablation system showed no marked change with increased applied force. Short 5 second application duration generated consistent lesion dimensions across computational, thermochromic gel, and thigh models.

AFS 2021-12

\section{Uploaded File(s)}

\section{Lesion Morphology}

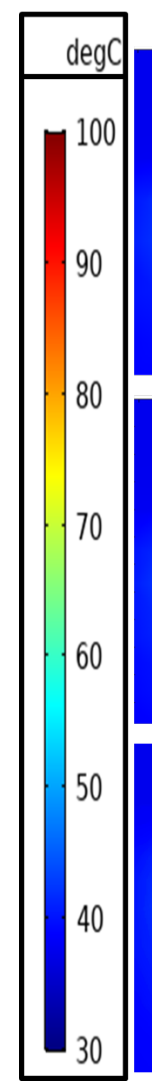

5 Seconds
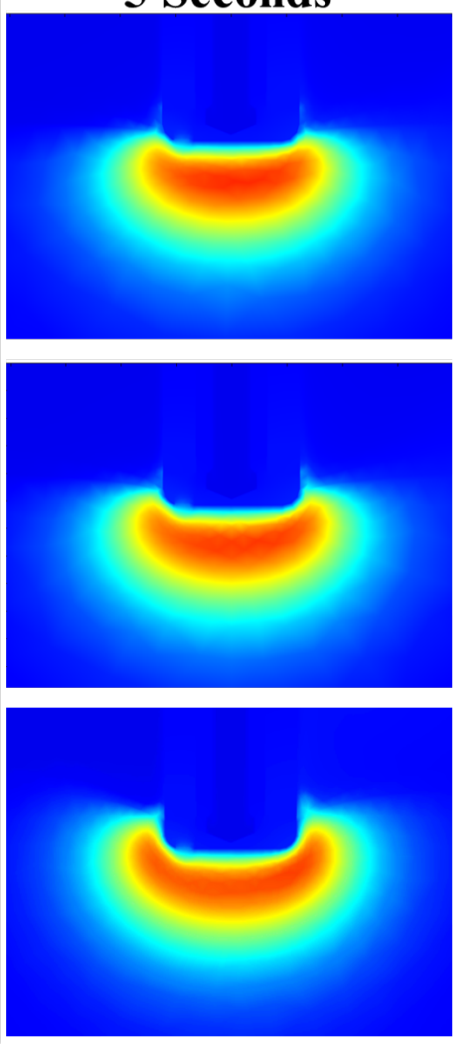

5 Seconds
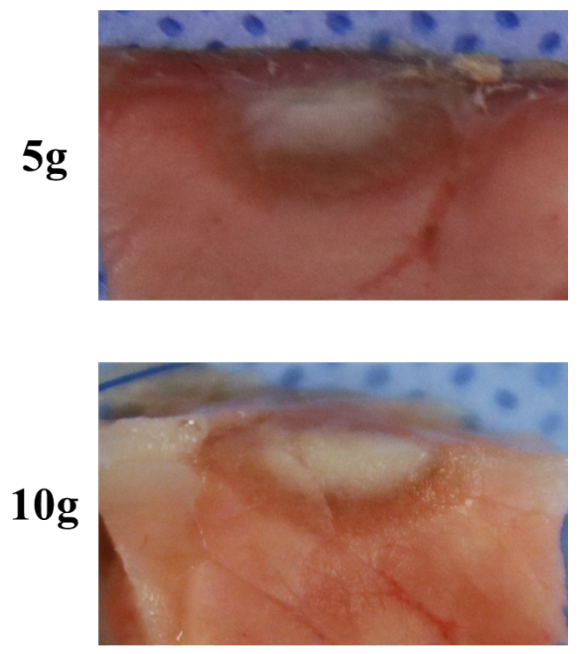

$30 \mathrm{~g}$

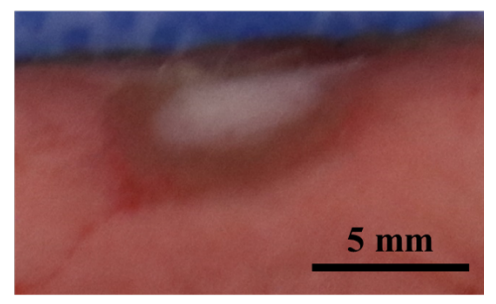

\section{AFS 2021-13}

Abstract Title: Near zero fluoroscopy in cryoballoon catheter ablation in patients with atrial fibrillation using the new KODEX-EPD imaging system

Author(s): Vincenzo Schillaci, Medicine and Surgery

Co-Author(s):

Alberto Arestia, Medicine

Gergana Shopova, Medicine

Giuseppe Stabile, Medicine

Francesco Solimene, Medicine

Casa di cura Montevergine 
Via M. Malzoni 1 Mercogliano

Introduction | Objectives: Fluoroscopy is commonly used in cryoballoon catheter ablation for pulmonary vein $(\mathrm{PV})$ isolation procedures in patients with atrial fibrillation $(\mathrm{AF})$. The purpose of this study was to verify the feasibility of a new dielectric imaging system in reducing the radiation exposure during cryoballoon ablation in patients with AF.

\section{Methods:}

We enrolled 26 consecutive patients with paroxysmal AF: 13 patients underwent the procedure under fluoroscopy guidance before the new system introduction, while 13 patient underwent the procedure under fluoroscopy and KODEX-EPD system (EPD Solutions, a Philips Company) guidance with its occlusion tool software. After transseptal access a detailed image reconstruction of left atrium and PVs was achieved with the Achieve (Medtronic Inc.) octapolar circular mapping catheter and the cryoablation was performed with the Arctic Front Advance (Medtronic Inc.) cryoballoon.

\section{Results:}

Total time of the procedure

was comparable between the two groups ( $90.15 \pm 28.67$ vs $80.77 \pm 17.17$ using KODEX-EPD, $\mathrm{p}=0.34$ ), while fluoroscopy time was significantly lower in the group using KODEX-EPD (16.92 \pm 8.96 vs $5.54 \pm 2.06$, p< 0.01). Acute isolation was achieved in all PVs. No 30 days complication was observed.

\section{Conclusions:}

This is the first study that demonstrates the feasibility of a reduce fluoroscopy workflow using the novel KODEX-EPD system in a cryoballoon procedure.

\section{AFS 2021-13}

\section{Uploaded File(s)}

Fluoroscopy time procedure between the two groups

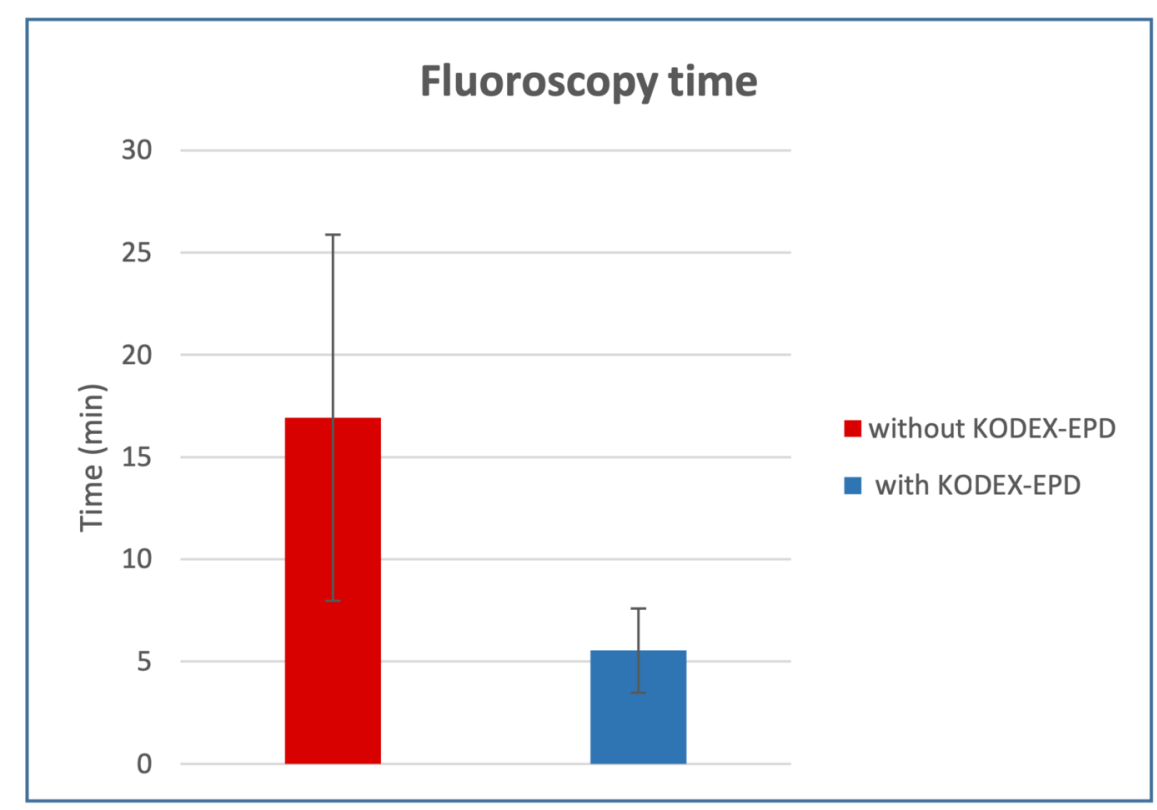

AFS 2021-14 
Abstract Title: How does the Level of Pulmonary Venous Isolation Compare Between Pulsed Field Ablation and Thermal Energy Ablation (Radiofrequency, Cryo or Laser)?

Author(s): Iwanari Kawamura, MD

Co-Author(s):

Petr Neuzil, MD

Poojita Shivamurthy, MD

Kenji Kuroki, MD,PhD

Jeff Lam, MS

Daniel Masikantow, MD

Edward Chu, MD

Mohit Turagam, MD

Kentro Minami, MD

Moritoshi Funasako, MD,PhD

Jan Petru, MD

Aamir Sofi, MD

Subbarao Choudry, MD

Marc Miller, MD

Noelle Langan, MD

William Whang, MD

Jacob Koruth, MD

Srinivas Dukkipati, MD

Vivek Reddy, MD

Ichan School of Medicine at Mount Sinai

One Gustave L. Levy Place, Box 1030, New York, NY, USA, 10029

Introduction | Objectives: Catheter-based pulsed field ablation (PFA) is a novel non-thermal energy source for pulmonary vein isolation (PVI) with unique aspects including reduced dependence on tissue contact, and enhanced safety. However its effect on the area of electrical PVI has not been described as compared to PVI using standard thermal ablation technologies.

Methods: In a clinical trial (NCT 03714178), paroxysmal atrial fibrillation (PAF) patients underwent PVI using an optimized biphasic waveform with a multielectrode pentaspline PFA catheter (Farapulse Inc). After 75 days, detailed voltage maps were created during prospective, protocol-specified remapping studies. In order to compare the area of PVI, we retrospectively collected consecutive PAF patients who i) underwent PVI using thermal energy, ii) underwent a redo procedure for recurrence, and iii) all PVs were durably isolated. After detailed electroanatomical mapping with a high-density mapping catheter, the left and right $\mathrm{PV}$ antral isolation areas and nonablated posterior wall areas were quantified.

Results: We identified 20 patients with durable PVI in the PFA cohort, and 39 patients from the thermal ablation cohort (29 radiofrequency ablation, 6 cryoballoon and 4 visually-guided laser balloon). PFA patients were younger and had shorter follow up compared to patients in the thermal cohort. Left atrial diameter and ventricular systolic function were preserved in both cohorts. There was no significant difference 
between the PFA and thermal ablation cohort in the left- and right-sided PV isolation areas, as well as nonablated posterior wall area. The PFA cohort has smaller right superior PV isolation area compared to the radiofrequency ablation cohort, but this significance disappeared after propensity score matching. Notch-like normal voltage areas was seen at the posterior side of the carina in the Balloon sub-cohort, but not the PFA or RFA cohorts.

Conclusions: Catheter-based PVI with the pentaspline PFA catheter creates a chronic PV antral isolation area as encompassing as thermal energy ablation.

AFS 2021-14

\section{Uploaded File(s)}

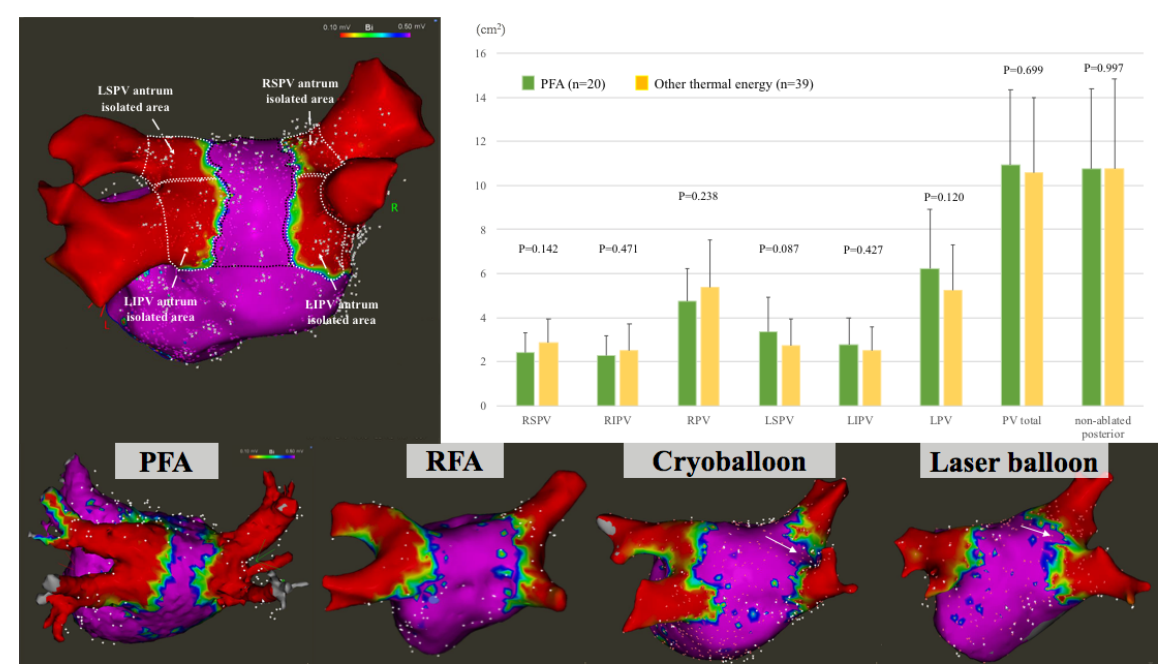

\section{AFS 2021-15}

Abstract Title: Treatment of Left Atrial Occluder Leak: Embolic Coils as a Potential Therapeutic Strategy Author(s): Daniel Musikantow, MD

Co-Author(s):

Poojita Shivamurthy, MD - Icahn School of Medicine at Mount Sinai

Lori Croft, MD

Iwanari Kawamura, MD

Mohit Turagam, MD

William Whang, MD

Srinivas Dukkipati, MD

Martin Goldman, MD - Icahn School of Medicine at Mount Sinai

Vivek Reddy, MD

Icahn School of Medicine at Mount Sinai

One Gustav Levy Place New York, NY 10029 
Introduction | Objectives: Left atrial appendage closure (LAAC) has proven to be an effective alternative to long term oral anticoagulation in the prevention of thromboembolic events in patients with atrial fibrillation and elevated $\mathrm{CHA}_{2} \mathrm{DS}_{2}$-VASc. In some patients, inadequate seal may result in persistent peri-device flow and inability of the appendage to fully thrombose - thereby representing a potential source for thromboembolism. The objective of this abstract is to study the use of endovascular coiling of the appendage to address persistent peri-device leak in patients who have undergone appendage closure with the Watchman device.

Methods: This is a retrospective, single-center analysis involving patients who underwent placement of a Watchman LAAC device and returned for endovascular coiling to address persistent device leak between 2018-2020. Baseline characteristics, procedural outcomes and follow-up echocardiograms were analyzed to demonstrate the feasibility and safety of this technique. Clinical outcomes were determined from review of available chart documentation and follow up.

Results: Patients $(\mathrm{N}=20)$ were identified with a mean leak size of $3.8 \pm 1.3 \mathrm{~mm}$ (range $2.5-7 \mathrm{~mm}$ ) all of whom had a non-thrombosed appendage. Acute procedural success was achieved in $95 \%$ of patients. At eight-week follow up imaging, complete or significant reduction in flow beyond the appendage was achieved in $61 \%$ and $33 \%$ of patients respectively. The one procedure-related adverse event was a pericardial effusion prior to coil deployment, requiring percutaneous drainage. After a mean follow up period of $16 \pm 5.4$ months, $85 \%(\mathrm{~N}=17)$ of patients remained off of anticoagulation.

Conclusions: Identifying those at risk for thromboembolic events resulting from peri-device leak postWatchman implantation is a matter of continuing investigation. However, appendage coiling represents a new therapeutic tool to address this potential source for thromboembolism. Further studies are required to address the clinical impact of this technique, including the safety of discontinuing anticoagulation after successful coiling.

\section{AFS 2021-15}

Uploaded File(s)

Fluoroscopic Images of Coiling Procedure 

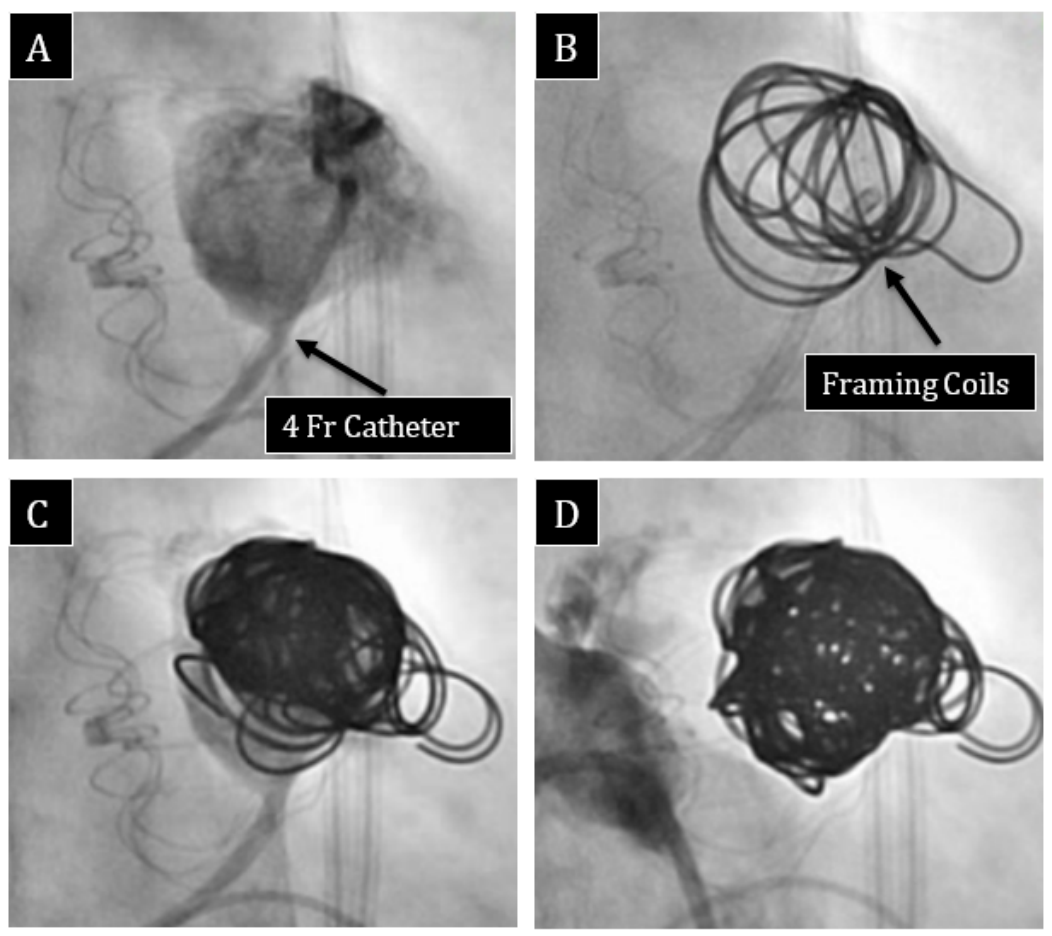

\begin{abstract}
A: Contrast injection from $4 \mathrm{Fr}$ catheter behind the LAAC demonstrating a patent appendage. B: Framing coils deployed to outline the LAA. C: Hydrocoils deployed until LAA occupied. D: Contrast injection at conclusion of procedure showing fully embolized appendage.
\end{abstract}

\begin{abstract}
AFS 2021-16
\end{abstract}
Abstract Title: Comparison of Fluoroscopy Exposure in Tacticath SE Ablation Catheter versus in Tacticath Ablation Catheter

Author(s): Alexander D. Sweeting, BS Biology

\title{
Co-Author(s):
}

Kent Nilsson, Doctor of Medicine - Cardiac Electrophysiology, Johns Hopkins University

MIchael Ahmed, BS Cellular Biology - Medical Student

Sujay Sreenivasan, A.B Music - Medical Student, University of Georgia

Medical College of Georgia

AU/UGA MEDICAL PARTNERSHIP University of Georgia Health Sciences Campus Prince Avenue Athens, GA 30602

Introduction | Objectives: Pulmonary vein isolation (PVI) has emerged as a cornerstone of management for atrial fibrillation. This procedure typically includes the use of fluoroscopy studies for catheter navigation, thus exposing both health care workers and the patient to harmful ionizing radiation. We hypothesized that the next generation Tacticath SE ablation catheter would reduce fluoroscopy time compared with the previously used Tacticath ablation catheter due to its ability to be visualized using Mediguide virtual fluoroscopy. 
Methods: Retrospective analysis of patients who underwent PVI at Piedmont Athens Regional Hospital over a three year period (06/15/2017 to 06/25/2020). 140 patients were ablated using a Tacticath ablation catheter, and 85 patients were ablated using the sensor enabled Tacticath SE. The average fluoroscopy study time between the two cohorts were subsequently compared.

Results: The average fluoroscopy time using the Tacticath ablation catheter was 4.96 minutes versus 3.6 minutes with the next generationTacticath SE ablation catheter $(27.4 \%$ reduction, $\mathrm{p}<0.001)$.

Conclusions: n combination with Mediguide Virtual Fluoroscopy, the Tacticath SE ablation catheter significantly reduces fluoroscopy study time and ionizing radiation in PVI procedures.

AFS 2021-17

Abstract Title: Cryoballoon Pulmonary-Vein Isolation is Effective and Safe in Patients with Atrial Fibrillation and Heart Failure

Author(s): Andreas A. Boehmer, MD

Co-Author(s):

Moritz Rothe, MD

Alena Summ, MD

Schukria Jaqubi, MD

Jaber Abboud, MD

Bianca Dobre, MD

Christina Soether, MD

Bernhard Kaess, PD MD

Joachim Ehrlich, Prof Dr

St. Josefs-Hospital Wiesbaden GmbH

Beethovenstraße 2065189 Wiesbaden

Introduction | Objectives: Atrial fibrillation (AF) and heart failure (HF) are both of increasing prevalence and mortality. There is evidence that ablation treatment can improve the prognosis of this particular patient subentity but prospective data regarding efficacy and safety of cryoballon pulmonary-vein isolation (cryoPVI) in patients with HF are lacking. The objective was to prospectively compare cryoPVI in patients without and with HF (LVEF [?] 40\%) with respect to efficacy and safety.

Methods: We analyzed consecutive patients who underwent cryoPVI in a single-center cohort between 2018 and 2020. Ablations were performed in a standardized fashion. Follow-up was performed at 3, 6, 12, 18 and 24 months after cryoPVI . Endpoints were: symptomatic AF relapse for efficacy and bleeding, phrenic nerve injury, stroke or death for safety. Statistical analysis was performed by log rank test and Kaplan-Meier method to estimate 12-month event-rate.

Results: We included 464 patients into the analysis, of these 414 had normal LVEF (age 67+-10 years, CHA2DS2-VASc Score 2.6+-1.5, 54\% male) while 50 patients (10.8\%) suffered from HF (age 69+-11 years, CHA2DS2-VASc Score 2.7+-1.5, 55\% male). Relapse rates were similar for patients with and without HF ( $67.5 \%$ vs. $74.4 \%, \mathrm{P}=0.41$, Figure). Primary safety end point occurred in form of pericardial effusion or transient phrenic nerve injury in six patients with normal LVEF (1.6\%) and one with HF (2\%). No deaths or strokes were observed. 
Conclusions: CryoPVI is an effective and safe ablation procedure for patients suffering from AF and HF. If there is prognostic benefit from cryoPVI in this context needs to be addressed in prospective trials.AFS 2021-17

\section{Uploaded File(s)}

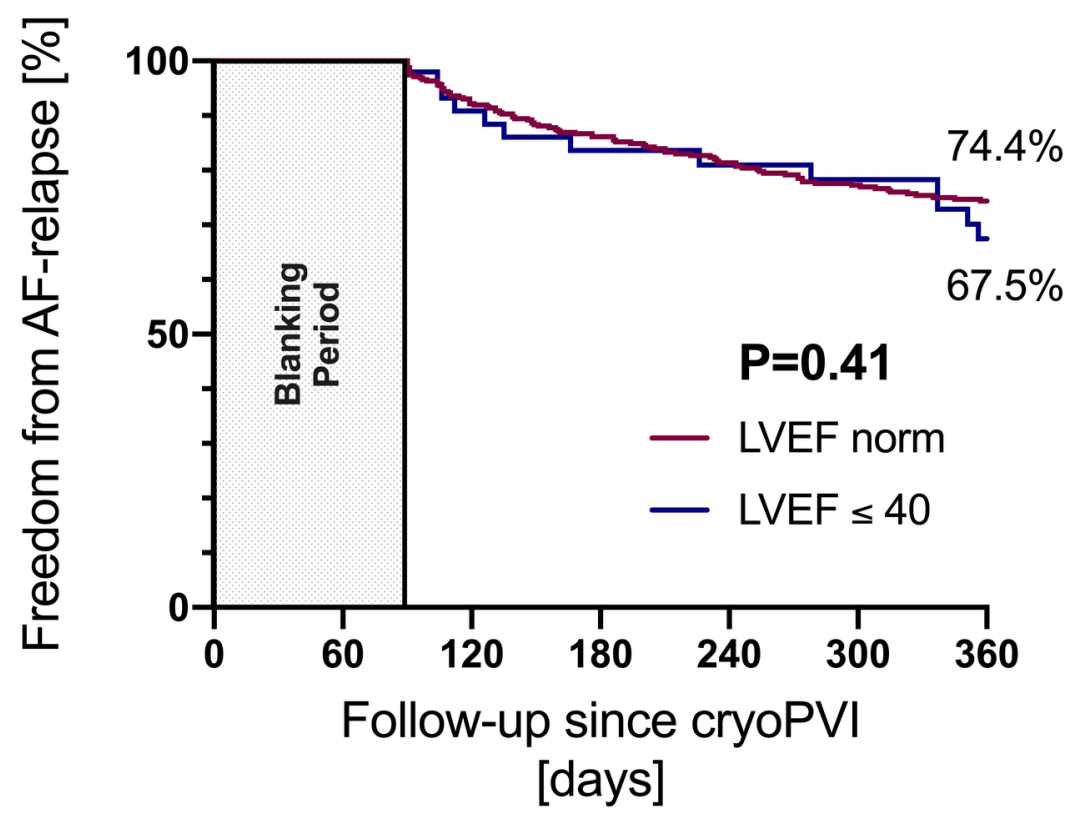

AFS 2021-18

Abstract Title: Decreased noise and identification of very low voltage signals using a novel electrophysiology recording system

Author(s): Josef Kautzner, MD, PhD

Co-Author(s):

Jana Haskova, MD - electrophysiologist, Institute for Clinical and Experimental Medicine (IKEM)

Predrag Stojadinovic, MD - electrophysiologist, Institute for Clinical and Experimental Medicine (IKEM)

Petr Peichl, MD, PhD - Director of EP labs, Institute for Clinical and Experimental Medicine (IKEM)

Institute for Clinical and Experimental Medicine (IKEM)

Videnska 1958/9, 14021 Prague, Czech Republic

Introduction | Objectives: Current electrophysiology (EP) recording systems may exhibit noise, artifacts and interference that obscure clinically important low voltage electrograms (EGMs). Furthermore, their reliance on $50 \mathrm{~Hz} / 60 \mathrm{~Hz}$ notch filtering may distort EGMs, impacting interpretation. We compared recordings from conventional EP recording systems to those from a novel system designed to reduce noise and minimize filtering.

Methods: EGMs were recorded simultaneously from nine patients undergoing ablation using conventional diagnostic, mapping and ablation catheters, with the CathVision Cube ${ }^{\mathrm{TM}}$ EP System (CathVision, Copenhagen, Denmark) in parallel with the GE CardioLab (Control) and one of two 3D mapping systems (Carto3 
or Ensite). EGMs were recorded using Wilson Central Terminal, filtering between $0.05-500 \mathrm{~Hz}$, with and without notch filters. One minute periods of unfiltered unipolar signals from baseline and during RF ablation were analyzed in 10-second windows for the spectral power density of powerline noise and signal saturation. Control notch-filtered unipolar and bipolar EGM morphology and peak amplitude were compared to that of Cube unfiltered unipolar EGMs. Furthermore, the operator evaluated the compatibility of the Cube with the 3D mapping systems and catheters.

Results: At $50 \mathrm{~Hz}$ the mean Cube power was 6.1 times lower than Control. Saturation artifact was observed during every Control but no Cube ablations. Notch-filter induced EGM morphology changes (reduced amplitude, phase distortion, and artificial peaks) were observed in five Control but no Cube recordings (Fig). The Cube was compatible with both conventional 3D mapping systems and commercially available catheters.

Conclusions: CathVision Cube system EGM recordings were associated with fewer noise and signal artifacts, than the conventional systems. The notch filtering required to reduce conventional system noise distorted EGMs causing EGM fractionation that was not present on unfiltered Cube unipolar EGMs. The CathVision Cube is a novel EP recording system that provides EGM data not apparent on conventional systems, which may be clinically important.

\section{AFS 2021-18 Uploaded File(s)}

\section{An example of recordings}

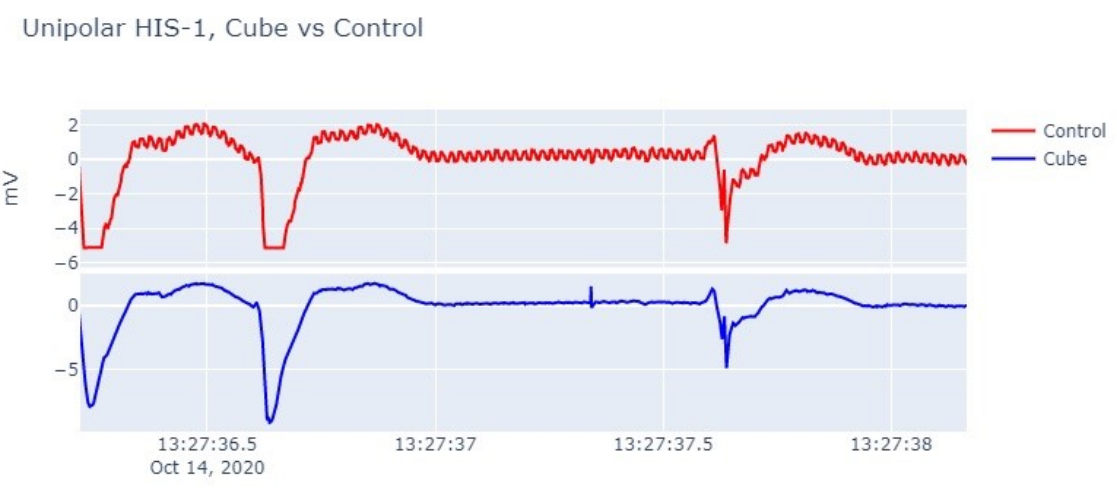

AFS 2021-19

Abstract Title: Barriers and Financial Impact of Same-Day Discharge after Atrial Fibrillation Ablation

Author(s): Edward Chu, MD

Co-Author(s):

Chi Zhang, MD

Mohit Turagam, MD

Noelle Langan, MD

Aamir Sofi, MD

Subbarao Choudry, MD

Georgios Syros, MD

Marc Miller, MD 
Jacob Koruth, MD

William Whang, MD

Srinivas Dukkipati, MD

Vivek Reddy, MD

Icahn School of Medicine at Mount Sinai

1468 Madison Avenue, New York, NY 10029

Introduction | Objectives: Although post-procedure care after catheter ablation for atrial fibrillation (AF) has historically necessitated overnight stay, same-day discharge (SDD) after the ablation procedure is feasible and increasingly being considered. This study examined the barriers and financial impact associated with SDD in a contemporary cohort of patients undergoing elective AF ablation.

Methods: A single center retrospective review was conducted of all first case-of-the-day outpatient AF ablations performed in 2019. The primary outcome was the proportion of patients that could have undergone SDD. Barriers to discharge were defined as any non-routine intervention that prevented SDD. The financial impact of SDD was based on savings from avoidance of the overnight hospital stay and revenue related to management of chest pain facilitated by a vacant hospital bed.

Results: Of the 249 patients who underwent AF ablation, SDD could have occurred in 157 patients (63\%) without change in management and in up to 200 patients (80\%) if avoidable barriers were addressed. Barriers to SDD included non-clinical logistical issues (43\%), prolonged post-procedure recovery $(42 \%)$ and minor procedural complications (15\%), as shown in the figure. The financial gain from SDD would have ranged from $\$ 1,108,692$ (assuming discharge of $63 \%$ of eligible patients) to $\$ 1,478,256$ (assuming $80 \%$ discharge) over the course of a year.

Conclusions: Up to $80 \%$ of patients undergoing outpatient AF ablation were amenable to SDD if avoidable delays in care had been anticipated. Based on reduced hospital operating expenses and increased revenue from management of individuals with chest pain, this would translate to a financial savings of $~ \$ 1.5$ million.

\section{AFS 2021-19}

Uploaded File(s)

Barriers to discharge 


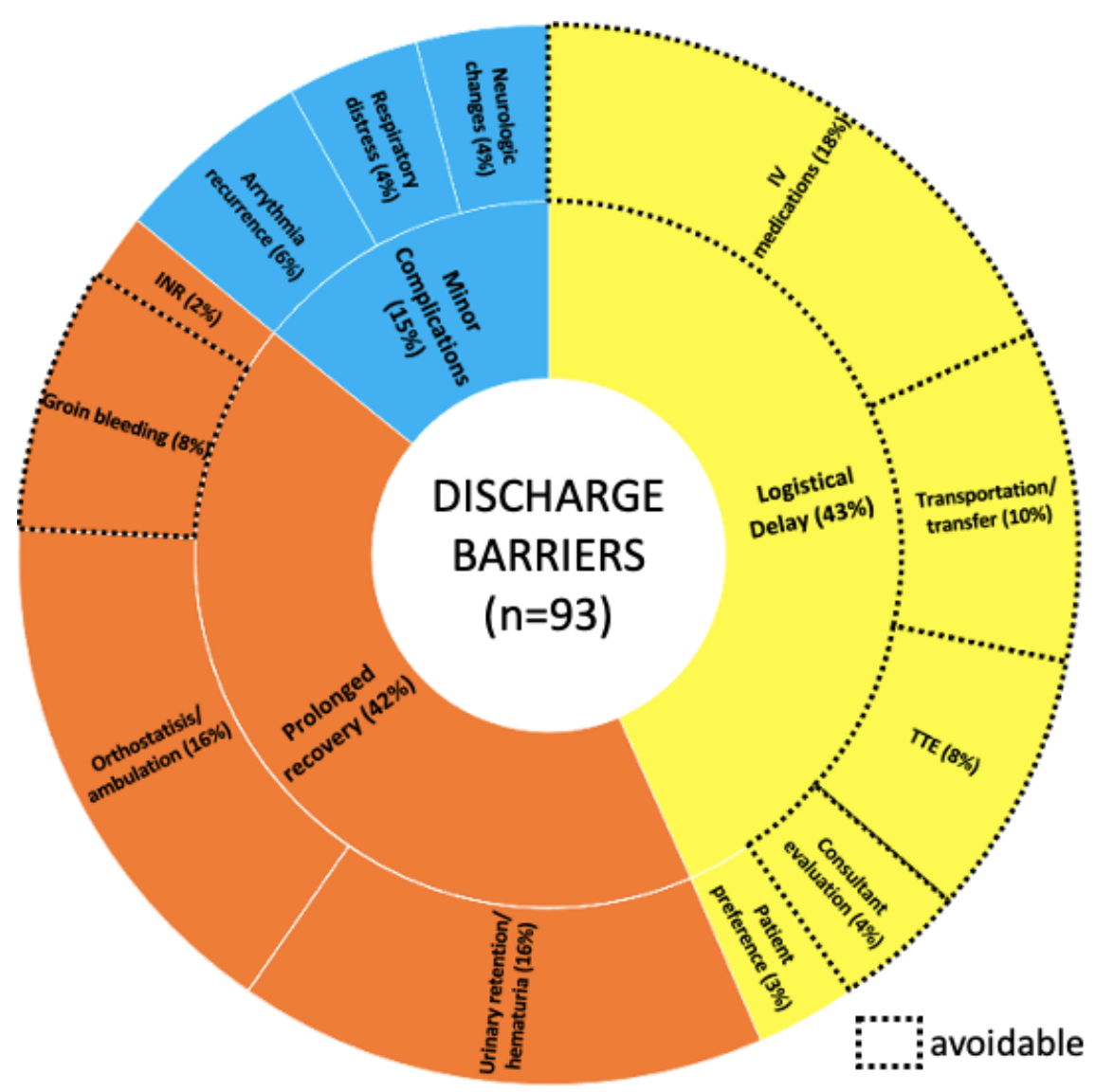

AFS 2021-20

Abstract Title: Outcomes of Hybrid Convergent procedure for the treatment long-standing persistent atrial fibrillation

Author(s): Nitesh Sood, MD

Co-Author(s):

Christopher Zambrano, MD - Southcoast Health System

Andrew Sherman, MD - Tampa Cardiac Specialists

Kevin Makati, MD - Tampa Cardiac Specialists

Southcoast Health System

363 Highland AVE, Fall River, MA 02127

Introduction | Objectives: : In long-standing persistent AF (LSPAF) patients, sustenance of AF is mediated by extra pulmonary vein (PV) drivers. Left atrial posterior wall is a desirable target however the results with endocardial ablation alone are limited due to challenges of achieving transmural lesions while maintaining safety. Combined endocardial and epicardial ablation may provide improved outcomes.

Methods: : A total of 105 consecutive symptomatic drug refractory LSPAF patients underwent hybrid procedure with EPi-Sense RF device for epicardial PV and posterior wall ablation and cryoballoon for endocardial PV isolation. A right sided cavo-tricuspid isthmus line was created with endocardial RF. The freedom from AF/AFL/AT was assessed through 12mo and 18mo using Kaplan-Meier analysis. In patients 
who received continuous monitoring (62\% with holter/implantable devices), residual AF burden reduction was assessed

Results: The mean AF duration was 4.9 years, $71 \%$ had previous cardioversion and $55 \%$ failed catheter ablation. The effectiveness results are summarized in the table below. No deaths, atrioesophageal fistula, stroke, or cardiac perforation occurred. Four patients (3.8\%) experienced pericardial effusions, 1 (1\%) had excessive bleeding and $1(1 \%)$ had volume overload.

Conclusions: Hybrid Convergent procedure is safe and effective for the treatment of long-standing persistent $\mathrm{AF}$ patients who currently do not have an FDA approved alternative

AFS 2021-20

Uploaded File(s)

Outcomes of Hybrid Convergent procedure for the treatment long-standing persistent atrial fibrillation

\begin{tabular}{|c|c|c|}
\hline Freedom from atrial arrhythmia & 12 months (95\% Cl) & 18 months $(95 \% \mathrm{Cl})$ \\
\hline Absent new AADs & $\begin{array}{c}76.8 \% \\
(67.2 \%, 84.0 \%) \\
\end{array}$ & $\begin{array}{c}62.4 \% \\
(50.8 \%, 72.0 \%) \\
\end{array}$ \\
\hline Absent AADs & $\begin{array}{c}68.8 \% \\
(58.8 \%, 76.8 \%)\end{array}$ & $\begin{array}{c}56.5 \% \\
(45.4 \%, 66.2 \%)\end{array}$ \\
\hline Regardless of AADs & $\begin{array}{c}80.3 \% \\
(70.8 \%, 87.0 \%)\end{array}$ & $\begin{array}{c}70.7 \% \\
(59.2 \%, 79.5 \%)\end{array}$ \\
\hline Off Amiodarone & $\begin{array}{c}75.8 \% \\
(66.0 \%, 83.1 \%)\end{array}$ & $\begin{array}{c}66.5 \% \\
(55.1 \%, 75.6 \%)\end{array}$ \\
\hline \multicolumn{3}{|l|}{ AF Burden } \\
\hline$<5 \%$ residual AF burden & $\begin{array}{c}89.7 \% \\
(81.6 \%, 94.3 \%)\end{array}$ & $\begin{array}{c}77.6 \% \\
(66.3 \%, 85.5 \%)\end{array}$ \\
\hline$<1 \%$ residual AF burden & $\begin{array}{c}87.5 \% \\
(79.0 \%, 92.7 \%)\end{array}$ & $\begin{array}{c}73.8 \% \\
(62.2 \%, 82.4 \%)\end{array}$ \\
\hline
\end{tabular}

\section{AFS 2021-21}

Abstract Title: Patient and Procedural Predictors of Atrial Arrhythmia Recurrence Post Diamond Tip Radiofrequency Ablation: Analysis from the DIAMOND-AF Trial

Author(s): H. Thomas McElderry, MD

Co-Author(s):

Jean-Paul Albenque, MD, PhD

Andrea Natale, MD

William Maddox, MD

Frank Cuoco, MD

Petr Neuzil, MD, PhD

Herve Poty, MD

Michael Getman, MS

Shufeng Liu, MS

Zdenek Starek, MD, PhD

Srinivas Dukkipati, MD

B. Judson Colley, MD

Amin Al-Ahmad, MD 
Darren Sidney, MD

Josef Kautzner, MD, PhD

University of Alabama Birmingham

Cardiology Clinic at The Kirklin Clinic of UAB Hospital 2000 6th Avenue South, Floor 4 Birmingham, AL 35233

Introduction | Objectives:Background: Cardiac ablation via radiofrequency (RF) energy has become the prominent method of treatment for patients with atrial fibrillation (AF). DiamondTemp Ablation (DTA) is a novel open irrigated temperature-controlled diamond tip RF ablation system which is designed to accurately assess tip-tissue temperatures and modulate power to maintain a target temperature during ablation. Presently, data are limited on patient and procedural characteristics associated with efficacy following pulmonary vein isolation with DTA.

Methods:Methods: We retrospectively analyzed the baseline characteristics and procedural data of the DTA arm from the DIAMOND-AF trial (NCT03334630). Univariate and multivariate Cox regression models were used to identify predictors of effectiveness failures.

Results:Results: 239 paroxysmal AF subjects were enrolled in the DTA arm (age $62 \pm 11$ years, 56.9\% male, left atrial diameter $4.0 \pm 0.6 \mathrm{~cm}$ ). 12-month freedom from AF was experienced by $79.1 \%$ of the DTA subjects. Univariate statistical testing of baseline patient characteristics revealed that duration of AF (HR, $1.05 ; 95 \% \mathrm{CI}, 1.00-1.10 ; \mathrm{p}=0.042)$ and vascular disease (HR, 3.55; 95\% CI, 1.51-8.35; $\mathrm{p}=0.004$ ) were predictors of atrial arrhythmia recurrence. By multivariate analysis, vascular disease was the only independent baseline characteristic predictor of recurrence. Regarding procedural characteristics, univariate testing revealed that a lower average temperature (HR, 1.12; 95\% CI, 1.03-1.23; $\mathrm{p}=0.009)$, use of conscious sedation ( $\mathrm{HR}, 1.85$; $95 \% \mathrm{CI}, 1.05-3.26 ; \mathrm{p}=0.033$ ), and the lack of esophageal temperature monitoring (HR, 2.34; 95\% CI, 1.30$4.20 ; \mathrm{p}=0.005)$ were predictors of atrial arrhythmia recurrence. By multivariate analysis, lower average temperature and lack of esophageal temperature monitoring were independent procedural characteristic predictors of recurrence.

Conclusions: Conclusion: Primary effectiveness was adversely impacted by the presence of vascular disease. When assessing the DTA procedural characteristics, effectiveness was negatively associated with lower average temperatures and absence of esophageal temperature monitoring.

\section{AFS 2021-21}

\section{Uploaded File(s)}

DAF Trial Predictors of Recurrence - Univariate Analysis 


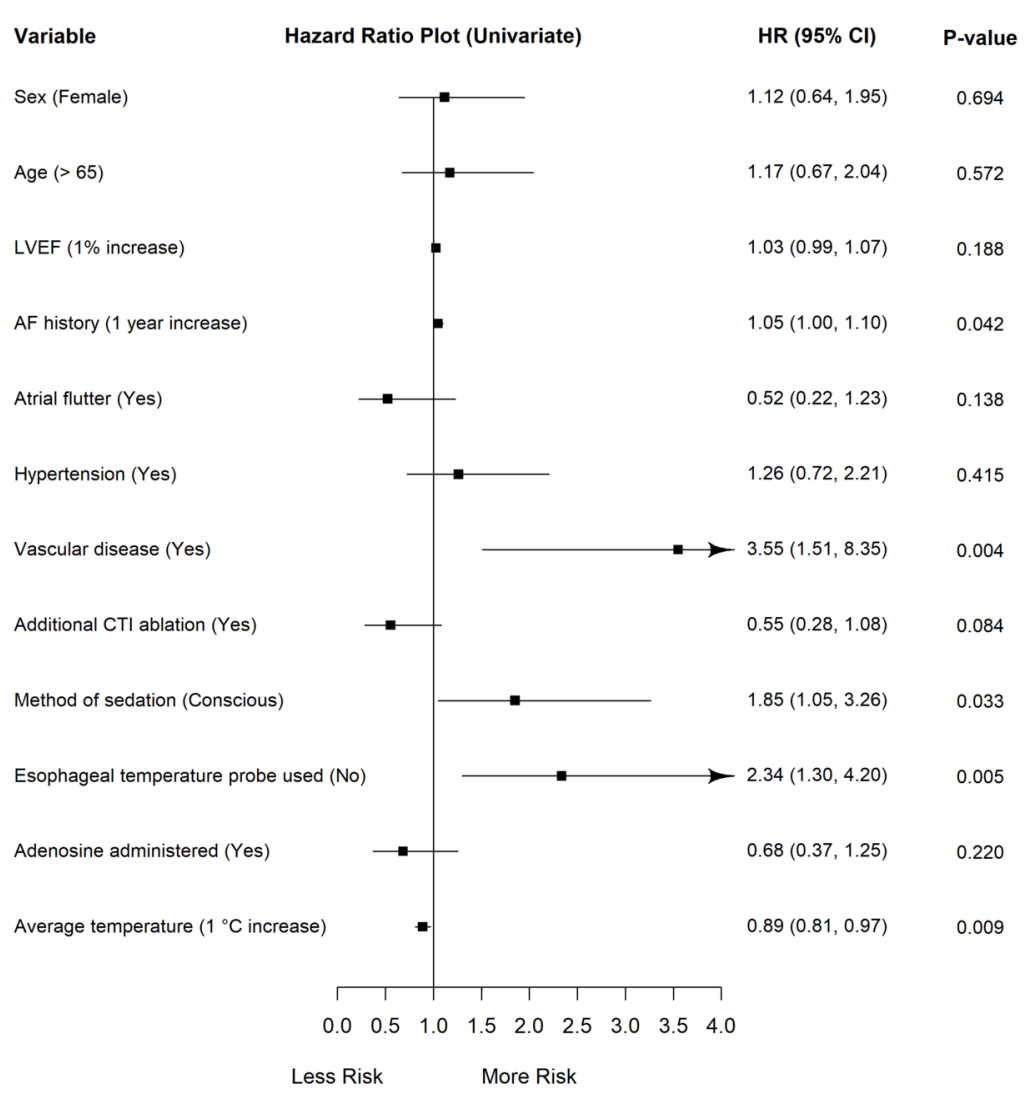

\section{AFS 2021-22}

Abstract Title: Learning Curve of a Novel Diamond Tip Ablation System and Relationship to Safety and Efficacy: Analysis from the DIAMOND-AF Trial

Author(s): H. Thomas McElderry, MD

\section{Co-Author(s):}

Jean-Paul Albenque, MD, PhD

Andrea Natale, MD

William Maddox, MD

Frank Cuoco, MD

Petr Neuzil, MD, PhD

Herve Poty, MD

Getman Michael, MS

Shufeng Liu, MS

Zdenek Starek, MD, PhD

Srinivas Dukkipati, MD 
B. Judson Colley, MD

Amin Al-Ahmad, MD

Darren Sidney, MD

Josef Kautzner, MD, PhD

University of Alabama Birmingham

Cardiology Clinic at The Kirklin Clinic of UAB Hospital 2000 6th Avenue South, Floor 4 Birmingham, AL 35233

Introduction | Objectives:Background: Radiofrequency (RF) catheter ablation has been a mainstay for the treatment of patients with atrial fibrillation $(\mathrm{AF})$. Because of the prevalence of $\mathrm{AF}$ and desire for treatment improvement, new technologies are introduced regularly. The learning curve for these new advancements and its potential impact on outcomes is widely appreciated but seldom reported. Objective: The DiamondTemp Ablation (DTA) system is a novel diamond tip externally irrigated RF ablation catheter that offers accurate tissue-temperature monitoring with real-time energy modulation. We sought to examine the learning curve of the temperature-controlled irrigated RF ablation system.

Methods:Methods: We assessed the DTA procedural data from the DIAMOND-AF trial (NCT03334630). Four time-oriented components of the procedure were evaluated as part of the learning curve assessment: total procedure time, left atrial (LA) dwell time, total RF time, and individual ablation duration.

Results:Results: A total of 235 subjects were treated with the DTA system at 23 sites in Europe, United States, and Canada. 140 subjects were treated within procedures 1-10 at a hospital (Group A) and 95 were treated during procedures 11-30 (Group B). Total procedure time, LA dwell time, total RF time, and individual ablation duration were all significantly shorter in Group B as compared to Group A (p-values < 0.05). Group A primary effectiveness success rate was 78\% (109 of 140), which did not differ against the $80 \%$ (76 of 95 ) seen in Group B, $\mathrm{p}=0.75$. Similarly, the primary safety event rates seen between Group A and Group B were not different ( $3 \%$ vs $4 \%, \mathrm{p}=0.72)$.

Conclusions:Conclusion: Investigators experienced a short learning curve with the DiamondTemp Ablation system with procedural efficiencies realized for the temperature-controlled system after the first 10 cases. This learning curve on procedure efficiency showed no impact on the safety or effectiveness results during the DIAMOND-AF trial.

AFS 2021-23

Abstract Title: Very high power, short duration pulmonary vein isolation with a temperature-controlled radiofrequency catheter: a prospective, multicenter study

Author(s): Jose Osorio, MD

Co-Author(s):

Ayman Hussein, MD - Cleveland Clinic Foundation

M. Craig Delaughter, MD - Texas Health Heart \& Vascular

George Monir, MD - Florida Hospital

Andrea Natale, MD - Texas Cardiac Arrhythmia Research

Srinivas Dukkipati, MD - Mount Sinai School of Medicine

Saumil Oza, MD - St Vincent's Medical Center

Emile Daoud, MD - Ohio State University Medical Center 
Luigi Di Biase, MD - Montefiore Hospital

Moussa Mansour, MD - Massachusetts General

Robert Fishel, MD - JFK Medical Center

Miguel Valderrabano, MD - Houston Methodist Research Institute

Kenneth Ellenbogen, MD - Virginia Commonwealth University

Grandview Medical Center

Grandview Medical Center Alabama Cardiovascular Group 3686 Grandview Parkway, Suite 720 Birmingham, $\mathrm{AL}$

Introduction | Objectives: The initial safety and feasibility of a novel temperature sensing catheter with microelectrodes and 6 thermocouples, used with both conventional power $(\mathrm{CP} ; 25-50 \mathrm{~W})$ and very high power short duration (vHPSD; $90 \mathrm{~W} / 4 \mathrm{~s}$ ) in temperature controlled modes for paroxysmal atrial fibrillation (PAF) ablation have previously been described. Longer-term clinical safety and effectiveness in a larger population has yet to be reported. Initial safety and interim $6 \mathrm{M}$ effectiveness of the vHPSD catheter in the treatment of symptomatic PAF are reported.

Methods: This prospective, non-randomized, multi-center study utilized vHPSD as the primary temperature control mode for PVI while the combination of vHPSD and CP could be used for PV touch-up or ablation outside of the PV. Primary safety endpoint was the incidence of any primary adverse events (PAEs) occurring within 7 days of the index procedure. Effectiveness was defined as freedom from documented atrial arrhythmia recurrence and freedom from additional 3 pre-defined primary effectiveness failure modes (acute failure, repeat ablation, new/higher dose AAD).

Results: A total of 191 PAF subjects were enrolled (mean age: $63.5 \pm 10.7$ years, $\mathrm{CHADS}_{2}$-VASc $2.4 \pm 1.5$, $60.7 \%$ male) across 22 sites in the United States. Of those enrolled, 166 subjects underwent ablation with the investigational catheter. Refer to the Table for the procedural characteristics. Mean maximum temperatures recorded remained similar in both modes (vHPSD: $45.0 \pm 5.3^{\circ} \mathrm{C}$; CP: $44.2 \pm 4.3^{\circ} \mathrm{C}$ ) with no esophageal injuries reported. Average applied contact force was similar between cases utilizing only vHPSD (15.8 $\pm 5.3 \mathrm{~g})$ and those that used a combination of vHPSD and CP (15.2 $\pm 5.3 \mathrm{~g})$ for ablation. Six patients (3.6\%) experienced PAEs (vascular access complication [3]; cardiac tamponade [2], and phrenic nerve injury [1]). At 6M postablation, freedom from effectiveness failure was $86.5 \%$.

Conclusions: vHPSD temperature controlled PAF ablation, in combination with CP, is highly efficient without compromising safety.

\section{AFS 2021-23}

Uploaded File(s)

Procedural Characteristics

Very high power, short duration pulmonary vein isolation with a temperature-controlled radiofrequency catheter_a prospective, multicenter study.xlsx

\begin{tabular}{l}
\hline Ablation Parameter (minutes) \\
\hline Total Procedure Time \\
Total Mapping Time \\
Total Fluoroscopy Time \\
RF Application Time \\
Total RF application time for ablating PVs \\
a RF application time was derived from the CARTO files for improved accuracy. All other procedural characteristics were \\
\hline
\end{tabular}




\section{AFS 2021-24}

Abstract Title: Successful Treatment of a $41 \mathrm{~mm}$ Left Common Pulmonary Vein Using the SecondGeneration Visually Guided Laser Balloon

Author(s): Richard P. Borge, Jr., M.D.

Co-Author(s):

Abington Memorial Hospital

1200 Old York Rd, Abington, PA 19001

Introduction | Objectives: Left common pulmonary veins (LCPV) are seen in roughly 10-20\% of the patient population with an increased prevalence in patients with AF as compared to the control. ${ }^{1,2}$ The average size of LCPVs has been recorded as $30 \pm 7 \mathrm{~mm}$, while, to date, the largest treated LCPV with the second-generation visually guided laser balloon (VGLB) is $38 \mathrm{~mm} .{ }^{3}$ Due to the large and variant size of LCPVs, they are frequently difficult to treat using common modalities. The VGLB allows for variable-sizing to accommodate various sizes and shapes of pulmonary veins (PV), such as LCPVs. We report on a case study of a $41 \mathrm{~mm}$ LCPV that was successfully treated through the utilization of variable balloon sizing with the second-generation VGLB.

Methods: N/A

Results: Patient presented for a redo AF ablation, having undergone a previous procedure using the Medtronic Cryoballoon. The patient's LCPV measured $41 \mathrm{~mm}$ on the computerized tomography scan. A pre-voltage map displayed electrical re-connection in the LCPV and right inferior PV. The superior branch of the long trunked LCPV was cannulated and the balloon was dynamically sized until the superior, anterior, and posterior aspects of the LCPV were displayed on the endoscopic image. The vein was ablated using various dosing (ranging from 5.5 Watts to 12 Watts) and a WACA "open 8" technique. ${ }^{4}$ Lower dosing was utilized on the inferior and superior aspect due to incomplete occlusion. The LCPV was successful electrically isolated on the first pass.

Conclusions: Variable-sizing and endoscopic visualization allowed for the safe and successful electrical reisolation of a $41 \mathrm{~mm} \mathrm{LCPV}$ without needing to segmentally isolate the vein.

\section{AFS 2021-24}

\section{Uploaded File(s)}

Left: CT scan of the left atrium showing a 41mm LCPV; Right: Endoscopic image of the LCPV as seen from the laser balloon

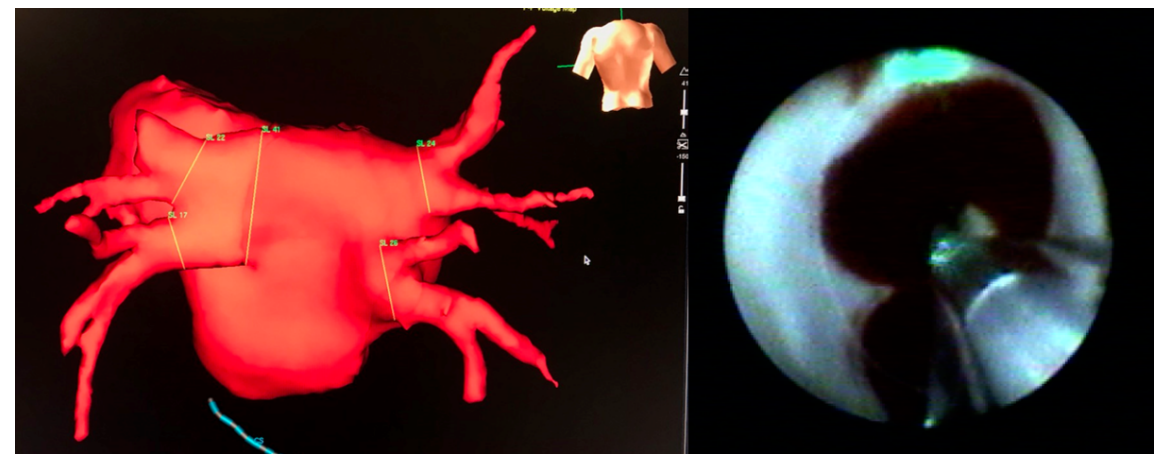

AFS 2021-25 
Abstract Title: Initial experience of novel dynamic mapping software using a high-density grid mapping catheter in the United States

Author(s): Monica Lo, MD

Co-Author(s):

Jose Martel, MD,MPH, FHRS - South Miami Hospital

Michael Bernard, MD, PhD - Ochsner Medical Center

Nicholas Olson, MD - Scripps Clinic and Prebys Cardiovascular Institute

Kevin Jackson, MD - Duke University Medical Center

Kevin Thomas, MD, FHRS - Duke University Medical Center

John Day, MD - Intermountain Medical Center

Sri Sundaram, MD, FHRS - South Denver Cardiology Associates

Toshimasa Okabe, MD - The Ohio State University Wexner Medical Center

Jonathan Piccini, MD, MHS, FACC, FAHA, FHRS - Duke University Medical Center

Caroline Tao, PhD - Abbott

Rajesh Venkataraman, MD - Houston Methodist The Woodlands

Arkansas Heart Hospital

7 Shackleford W Blvd, Little Rock, AR 72211

Introduction | Objectives: A novel dynamic mapping software became commercially available in the U.S. in July 2020. EnSite LiveView Dynamic Display utilizes the Advisor HD Grid Mapping Catheter to display beat-to-beat, dynamic regional mapping data. Procedural characteristics and clinical utilization of this novel software have not yet been reported from U.S. centers.

Methods: Procedural data were prospectively collected from over 30 operators in 18 U.S. centers during the first three months of commercialization Procedural characteristics recorded included procedure time, total RF time, workflow preference, and fluoroscopy time.

Results: A total of 119 cases were collected from July to September 2020. A steerable sheath was used with the mapping catheter in 63 cases (53\%). Double transseptal access was observed in 85 cases (71\%). LiveView Dynamic Display was used in a variety of cases including AF, VT, and atypical flutter. The EnSite AutoMap module was used in conjunction with the dynamic display in 50 cases (42\%). Visualization of real-time mapping data from the current location of the HD Grid mapping catheter was commonly used after traditional full-chamber maps. LiveView was also used as a pulmonary vein isolation (PVI) confirmation method in 49 cases $(41 \%)$. The most common reported usage of the LiveView Dynamic Display was PVI confirmation/gap identification (81\%), ablation line gap identification (58)\%, and identification of breakthrough activation (45\%)* note total exceeds $100 \%$, multiple usages per case were reported.

Conclusions: Initial experience in the U.S. demonstrated the diverse clinical utilization of LiveView Dynamic Display, including atrial and ventricular arrhythmias. The dynamic display of regional signals allows for rapid identification of ablation targets. When used during RF delivery, real-time assessment of regional activation patterns helped improve outcomes by rapidly identifying critical ablation location and ensuring successful lesion delivery. Further study which examines the impact of dynamic display on procedure workflow and efficacy may be warranted.

\section{AFS 2021-25}

Uploaded File(s) 


\section{Indication for mapping/ablation}

\begin{tabular}{|c|c|}
\hline Indication for mapping/ablation & Number of cases \\
\hline AVNRT & 1 \\
\hline AVRT & 2 \\
\hline \multicolumn{2}{|l|}{ Atrial flutter } \\
\hline atypical & 17 \\
\hline typical & 29 \\
\hline \multicolumn{2}{|l|}{ Paroxysmal AF } \\
\hline de novo & 47 \\
\hline redo & 19 \\
\hline \multicolumn{2}{|l|}{ Persistent AF } \\
\hline de novo & 14 \\
\hline redo & 21 \\
\hline \multicolumn{2}{|l|}{ VT } \\
\hline ischemic & 1 \\
\hline \multicolumn{2}{|l|}{ AT } \\
\hline focal & 6 \\
\hline left side & 5 \\
\hline right side & 1 \\
\hline Other & 1 \\
\hline
\end{tabular}

Note: total exceeds 119 cases, multiple indications per case were observed

\section{AFS 2021-26}

Abstract Title: Common settings and workflows incorporating dynamic mapping software using a highdensity grid mapping catheter

Author(s): Monica Lo, MD

\section{Co-Author(s):}

Jose Martel, MD,MPH, FHRS - South Miami Hospital

Michael Bernard, MD, PhD - Ochsner Medical Center

Nicholas Olson, MD - Scripps Clinic and Prebys Cardiovascular Institute

Kevin Jackson, MD - Duke University Medical Center

Kevin Thomas, MD, FHRS - Duke University Medical Center

John Day, MD - Intermountain Medical Center

Sri Sundaram, MD, FHRS - South Denver Cardiology Associates

Toshimasa Okabe, MD - The Ohio State University Wexner Medical Center

Jonathan Piccini, MD, MHS, FACC, FAHA, FHRS - Duke University Medical Center

Caroline Tao, PhD - Abbott

Rajesh Venkataraman, MD - Houston Methodist The Woodlands

Arkansas Heart Hospital 
7 Shackleford W Blvd, Little Rock, AR 72211

Introduction | Objectives: EnSite LiveView Dynamic Display utilizes the Advisor HD Grid Mapping Catheter to provide beat-to-beat, dynamic display of regional mapping data. In addition to the unique features offered by the mapping catheter, the new software comes with various settings that allow the users to customize the mapping display to accommodate different case types. The optimal settings and workflows to incorporate the dynamic data into routine ablation procedures have not been explored.

Methods: Procedural characteristics including map appearance setting, reference electrode, workflow preference, and ablation strategy were prospectively collected from over 30 operators in 18 U.S. centers during the first three months of commercialization.

Results: A total of 119 cases were collected. The majority (91\%) of the cases were indicated for mapping/ablation of atrial fibrillation (AF). The best duplicate algorithm was utilized in all cases with LiveView. AutoColor was used in 108 cases (91\%), and the CS catheter was selected as the reference electrode in 99 cases (83\%). Of note, 25 cases (21\%) also reported the use of the HD Grid Mapping Catheter as the reference electrode. This was mostly observed in de novo ablation cases where LiveView was used after traditional full-chamber maps. Common map appearance settings for interior and exterior projection, and interpolation was 5,7 , and 7 respectively. Voltage cutoff of $0.1 \mathrm{mV}$, range from 0.05 to $1.5 \mathrm{mV}$, was most frequently observed $(\mathrm{n}=53,45 \%)$ in this dataset for delineating scar. The use of dynamic display helped to identify areas that were under ablated in 88 cases $(74 \%)$. Out of 119 cases, $68 \%$ of the cases indicated that using LiveView did decrease the amount of mapping required to confirm ablation targets and that the desired endpoint had been achieved.

Conclusions: Initial procedure data suggests minimal workflow changes are required to incorporate the use of dynamic data in AF procedures. Adaptation of LiveView can help improve procedure efficiency and efficacy by reducing the need for full chamber maps and identifying areas that were under ablated, and confirming ablation endpoints. Further control study examining procedure efficiency and efficacy associated with dynamic mapping in AF may be warranted.

AFS 2021-26 Uploaded File(s)

Effect of LiveView Dynamic Mapping Display on mapping workflow

\begin{tabular}{|l|c|}
\hline $\begin{array}{l}\text { Did LiveView decrease the need for any of } \\
\text { the following? }\end{array}$ & Number of Cases (\%) \\
\hline Yes & $81(68 \%)$ \\
\hline Post Ablation Voltage/Timing Map & $22(18.5 \%)$ \\
\hline Voltage Map & $21(17.6 \%)$ \\
\hline LAT Map & $17(14.2 \%)$ \\
\hline Activation Map & $9(7.6 \%)$ \\
\hline Sparkle Map & $4(3.3 \%)$ \\
\hline Propagation Map & $3(2.5 \%)$ \\
\hline CFE Map & $4(3.3 \%)$ \\
\hline Other & $1(0.8 \%)$ \\
\hline Not Applicable/No Impact & $26(21.8 \%)$ \\
\hline
\end{tabular}

*CFE map-complex fractionated atrial electrogram

Table 1: Effect of LiveView Dynamic Mapping Display on mapping workflow

AFS 2021-27 
Abstract Title: A visualizable steerable sheath enables paroxysmal atrial fibrillation ablation with low or no fluoroscopy

Author(s): Jose Osorio, MD

Co-Author(s):

Gustavo Morales, MD

Tina Hunter, PhD

Paul Zei, MD

Joshua Silverstein, MD

Carolyn Whitmire, MBA

Allyson Varley, PhD, MPH

Anil Rajendra, MD

Grandview Medical Center

Grandview Medical Center Alabama Cardiovascular Group 3686 Grandview Parkway, Suite 720 Birmingham, AL

Introduction | Objectives: With advances in 3-dimensional (3D) electroanatomical mapping (EAM), fluoroscopy utilization and procedure time during paroxysmal atrial fibrillation (PAF) ablation have declined substantially. Recently, a new sheath became available that can be visualized with 3D EAM. This may further simplify ablation workflow and reduce fluoroscopy exposure. The objectives of this study were to evaluate early user experience incorporating the new EAM visualizable sheath into PAF ablations and to compare outcomes to procedures performed without the sheath.

Methods: Consecutive de novo PAF procedures using a porous tip contact force catheter at a high-volume site between January 2018 and May 2019 were included. Procedures using the new EAM visualizable sheath were compared to those performed without it. All ablations employed a standardized low-fluoroscopy workflow. Pulmonary vein isolation was performed with wide-area circumferential ablation, contact force was held between 10-20 grams, the catheter moved every 10-20 seconds, and radiofrequency (RF) energy set at 40-45 watts throughout the atrium. Due to asynchronous adoption of the sheath by operators of varying experience, analysis of outcomes employed stabilized inverse probability of treatment weights to balance cohorts by operator, age, sex, and $\mathrm{CHA}_{2} \mathrm{DS}_{2}$-VASc score.

Results: Cohorts were similar at baseline (Table). Complications were few across cohorts with no death or stroke. The EAM visualizable sheath cohort reported 7 complications: arteriovenous fistula (1), cardiac tamponade/pericardial effusion (2), hematoma (1), pseudoaneurysm (1), pericarditis (2) versus the without EAM visualizable sheath cohort reported 2 complications, cardiac tamponade/pericardial effusion and pseudoaneurysm. Use of the EAM visualizable sheath was associated with significant reduction in RF time and increase in ablations performed without fluoroscopy (Table). There were fewer reablations at 12 months in the EAM visualizable sheath cohort, but the difference was not statistically significant due to low number of events (Table).

Conclusions: A 3D EAM visualizable sheath safely reduced RF time and facilitated procedures performed without fluoroscopy, with no impact on clinical outcomes.

AFS 2021-27 Uploaded File(s)

Baseline Characteristics and Results 


\begin{tabular}{|c|c|c|c|}
\hline Baseline Characteristics (Unweighted) & $\begin{array}{c}\text { With EAM } \\
\text { Visualizable Sheath } \\
(\mathrm{N}=143)\end{array}$ & $\begin{array}{c}\text { Without EAM } \\
\text { Visualizable Sheath } \\
(\mathrm{N}=174)\end{array}$ & P-Value \\
\hline Age, years (mean, SD) & $64 \pm 12$ & $64 \pm 13$ & 0.7889 \\
\hline Sex $=$ Female & $78(54.5 \%)$ & $76(43.7 \%)$ & 0.0541 \\
\hline $\mathrm{CHA}_{2} \mathrm{DS}_{2}$-VASc Score (mean, SD) & $2.5 \pm 1.5$ & $2.6 \pm 1.6$ & 0.4698 \\
\hline Electrophysiologist Performing Ablation & & & $<0.0001$ \\
\hline Operator 1 & $112(78.3 \%)$ & $71(40.8 \%)$ & \\
\hline Operator 2 & $27(18.9 \%)$ & $61(35.1 \%)$ & \\
\hline Operator 3 & $4(2.8 \%)$ & $43(24.1 \%)$ & \\
\hline \multicolumn{4}{|c|}{ Results: Weighted by Inverse Probability of Treatment (by Operator, Age, Sex, CHA2DS2-VASc) } \\
\hline Ablations Performed with No Fluoroscopy & $98.6 \%$ & $91.9 \%$ & 0.0062 \\
\hline Total Procedure Time, minutes & $80.7 \pm 29.7$ & $80.1 \pm 34.3$ & 0.8746 \\
\hline Radiofrequency Time, minutes & $22.3 \pm 8.3$ & $26.6 \pm 10.1$ & $<0.0001$ \\
\hline Pulmonary Vein Ablation Time, minutes & $17.9 \pm 6.1$ & $19.0 \pm 6.6$ & 0.1571 \\
\hline \multicolumn{4}{|l|}{ First Pass Pulmonary Vein Isolation Success: } \\
\hline Left Pulmonary Veins & $82.2 \%$ & $85.5 \%$ & 0.4409 \\
\hline Right Pulmonary Veins & $66.1 \%$ & $64.5 \%$ & 0.7713 \\
\hline Reablations within 12 months & $3.9 \%$ & $6.2 \%$ & 0.3535 \\
\hline Single Procedure Freedom from Recurrence & $83.0 \%$ & $83.7 \%$ & 0.8717 \\
\hline
\end{tabular}

CHA2DS2-VASC = Congestive heart failure, Hypertension, Age $\geq 75$, Diabetes Mellitus, Stroke/Transient ischemic attack /

thromboembolism, Vascular disease, Age 65 to 74 years, Sex Category; EAM = electroanatomical mapping; SD = standard deviation;

\section{AFS 2021-28}

Abstract Title: The effect of dynamic mapping data on procedure efficiency in radiofrequency ablation of patients with atrial fibrillation

Author(s): Nicholas Olson, MD

\section{Co-Author(s):}

Monica Lo, MD - Arkansas Heart Hospital

Firas Zahwe, MD, FHRS - Ascension St. John Hospital

Arjun Gururaj, MD - Nevada Heart and Vascular Center

Jose Martel, MD,MPH, FHRS - South Miami Hospital

Michael Bernard, MD, PhD - Ochsner Medical Center

Caroline Tao, PhD - Abbott

Rajesh Venkataraman, MD - Houston Methodist The Woodlands

Scripps Clinic and Prebys Cardiovascular Institute

9898 Genesee Ave Fl 3 La Jolla, CA 92037

Introduction | Objectives: A new software module, EnSite LiveView Dynamic Display, utilizes the Advisor HD Grid Mapping Catheter to provide beat-to-beat, dynamic display of regional mapping data. Early clinical experience suggests that the combination of the two technologies may provide additional clinical benefit and improve procedure efficiency. However, comparative data has not been reported

Methods: Procedural data from experienced HD Grid mapping catheter users collected in the U.S. in 2019 were compared to procedure data collected from 17 U.S. centers during the first three months of commercialization of the new software module in 2020. Procedural characteristics, including procedure time, total RF time, fluoroscopy usage, and ablation strategy were compared. 
Results: A total of $87 \mathrm{HD}$ Grid cases without dynamic mapping (67.8\% paroxysmal AF and $32.2 \%$ persistent $\mathrm{AF}$ ) from 2019 were included in the analysis. Data from 103 cases (65.0\% paroxysmal AF and $35.0 \%$ persistent AF) were collected using HD Grid mapping catheter and the new dynamic mapping software. The ablation techniques and targets were similar between the two datasets. The total RF time and fluoroscopy time was shorter in both paroxysmal and persistent AF cases when the HD Grid mapping catheter was used together with LiveView Dynamic Display (Table 1). Compared to the historic dataset, the average procedure time was shorter in paroxysmal AF cases and comparable in persistent $\mathrm{AF}$ cases when dynamic data was available. Based on initial clinical experience during catheter ablation of AF, incorporating dynamic mapping data was proved beneficial to efficiency (mapping and ablation) and rapid gap identification (PVI and ablation lines).

Conclusions: Compared to the historic dataset, total RF time and fluoroscopy time was shorter when dynamic mapping was used during the case. Reduction in total RF time and fluoroscopy time can improve procedure safety by minimizing the creation of unnecessary lesions and exposure to radiation. The critical regions uncovered by dynamic mapping data may lead to better procedure outcomes and more durable lesions. Further controlled studies which examine the long term outcomes when dynamic mapping data is incorporated into the workflow may be warranted.

\section{AFS 2021-28}

\section{Uploaded File(s)}

\section{Comparison of procedure characteristics}

\begin{tabular}{|l|l|l|}
\hline Parosyxmal AF & HD Grid & HD Grid+LiveView \\
\hline RF Time(minutes) & $31.1 \pm 25.9$ & $21.2 \pm 14.5$ \\
\hline Fluoro Time (minutes) & $16.8 \pm 22.5$ & $4.3 \pm 3.5$ \\
\hline Procedure time (minutes) & $146.5 \pm 79.9$ & $132.8 \pm 68.1$ \\
\hline Persistent AF & HD Grid & HD Grid+LiveView \\
\hline RF Time(minutes) & $36.4 \pm 27.8$ & $20.5 \pm 12.7$ \\
\hline Fluoro Time (minutes) & $11.8 \pm 11.0$ & $8.5 \pm 9.5$ \\
\hline Procedure time (minutes) & $148.6 \pm 65.5$ & $154.1 \pm 49.8$ \\
\hline
\end{tabular}

\section{AFS 2021-29}

Abstract Title: Single Operator Acute Experience in a Community Setting Using the CardioFocus Heartlight X3 Endoscopic Ablation System - 75 Consecutive Patients with Post-Market Registry Data

Author(s): David N. Kenigsberg, MD

Co-Author(s):

Natalia Martin, CEPS, CCDS

Florida Heart Rhythm Specialists

1841 NE 45th Street, Ft. Lauderdale, FL 33308

Introduction | Objectives: The endoscopic ablation system (EAS) utilizes a laser balloon to provide pulmonary vein isolation (PVI) in patients with atrial fibrillation (AF). The X3 EAS is the newest generation employing a motor to provide an option for contiguous circumferential lesion delivery in the RAPID mode.

Methods: 75 consecutive AF patients were treated. After single transseptal puncture, a $12 \mathrm{~F}$ delivery sheath was positioned in the LA (left atrium). A 3D enabled circular mapping catheter was used to record PV 
(pulmonary vein) potentials and create an electroanatomic map of the LA. The EAS was inflated to obtain optimal circumferential contact with the PV antrum. Ideally, RAPID mode ablation was employed at 13$15 \mathrm{~W}$. In case of esophageal heating $>39^{\circ} \mathrm{C}$ or suboptimal tissue exposure, point-by-point ablation $(5.5-12 \mathrm{~W}$ for 20-30s) was used. During ablation of the right PVs, phrenic nerve pacing was performed via a diagnostic catheter. Single shot ablation was defined as one single RAPID energy application per PV to complete the singular, circular lesion set. PV conduction was re-assessed with 3D mapping after all PVs had been treated. In case of residual PV conduction, gap mapping followed by EAS guided ablation was performed. Procedure time was defined as initial groin puncture to withdrawal of the X3 EAS catheter. LA treatment time was defined by transseptal puncture to withdrawal of the X3 EAS catheter.

Results: 75 AF patients (68.1\% male, mean age $64.6+11.4$ years, $89.33 \%$ paroxysmal) with 298 PVs underwent X3 EAS ablation. Of the 298 PVs attempted, 298 (100\%) were isolated with X3 EAS and in $270 / 298(90.6 \%)$, PVs were isolated first pass. The median procedure time was $82.0(45.0,185.0)$ minutes with a median LA time of $69.0(36.0,173.0)$ minutes. The median fluoroscopy time was $6.0(1.1,17.6)$ minutes. No acute phrenic nerve injury, pericardial effusion or pericardial tamponade occurred in this cohort.

Conclusions: The X3 EAS catheter with RAPID mode achieved acute isolation in 100\% of all PVs with a $90.6 \%$ first pass isolation rate and a substantially faster procedure time (82.0 minutes) compared to previous EAS generations. Procedure, LA and fluoroscopy times decreased as user experience increased.

\section{AFS 2021-29 Uploaded File(s) Table 1}




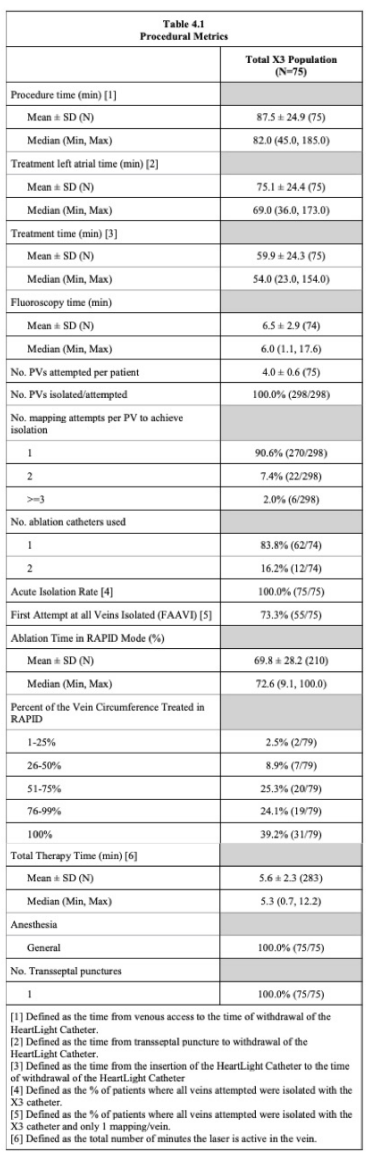

AFS 2021-30

Abstract Title: Initial multi-center experience using a RF transseptal wire for fluoroless left atrial access for RF and cryoballoon ablations

Author(s): Hany Demo, MD

\section{Co-Author(s):}

Mansour Razminia, MD - St. Joseph Hospital, Amita Health, Director of Clinical Cardiac Electrophysiology

Swedish Hospital part of NorthShore University

5140 N California Ave, Chicago, IL 60625

Introduction | Objectives: Fluoroscopy exposure and long-term lead wear have known health risks. Optimization of fluoroscope parameters, electroanatomic mapping (EAM) and intracardiac echocardiography (ICE) reduce radiation exposure, but transseptal puncture (TSP) and procedural efficiency remain barriers 
to full adoption. This is the first reported fluoroless clinical experience using a new RF transseptal system that offers more efficient left atrial (LA) access through reduced device exchanges

Methods: Fluoroless RF and cryoballoon ablations at 2 centers were retrospectively reviewed. TSP was performed using the VersaCross RF system (Baylis Medical) comprised of a transseptal sheath, shapeable dilator and RF wire (J-tip or pigtail) that can be used to perform TSP. The wire was also used to deliver the FlexCath sheath (Medtronic) in cryoballoon ablations. The RF wire tip was visualized as a discrete point on EAM. ICE was used to confirm wire, sheath and dilator location. Transseptal success, time, fluoroscopy use and procedural complications were evaluated

Results: Single (4) or double (19) TSP were performed $100 \%$ successfully with no fluoroscopy or lead use. Arrhythmias treated were: paroxysmal AF (10), persistent AF (8), long-standing persistent AF (2) and atrial tachycardia (4). Seven patients had prior ablation. TSP was achieved $14.2 \pm 6.0 \mathrm{~min}(\mathrm{n}=16)$ from procedure start or $3.5 \pm 2.2 \mathrm{~min}(\mathrm{n}=35)$ from $\mathrm{RF}$ wire insertion in the femoral vein. Visualization on ICE and EAM was achieved as the RF wire was advanced up to the SVC, sheath and dilator tracked over the wire, Wire pulled back, dropdown to the fossa ovalis with the wire exposed, and wire position in the LA to confirm TSP (Figure 1). No device exchanges were needed for TSP or repositioning on the septum after femoral access. There were no TSP-related complications. Subsequent RF (21) or cryoballoon (2) ablation was performed as per usual protocol

Conclusions: Our experience supports the feasibility of fluoroless TSP using the VersaCross RF system. Use of a multi-functional RF transseptal wire eliminated extra device exchanges and minimized the time to LA access. Larger comparative studies are needed to confirm safety and efficacy associated with the use of the RF transseptal wire

\section{AFS 2021-30}

Uploaded File(s)

Figure 1. Visualization of RF wire, sheath and dilator on EAM and ICE. 


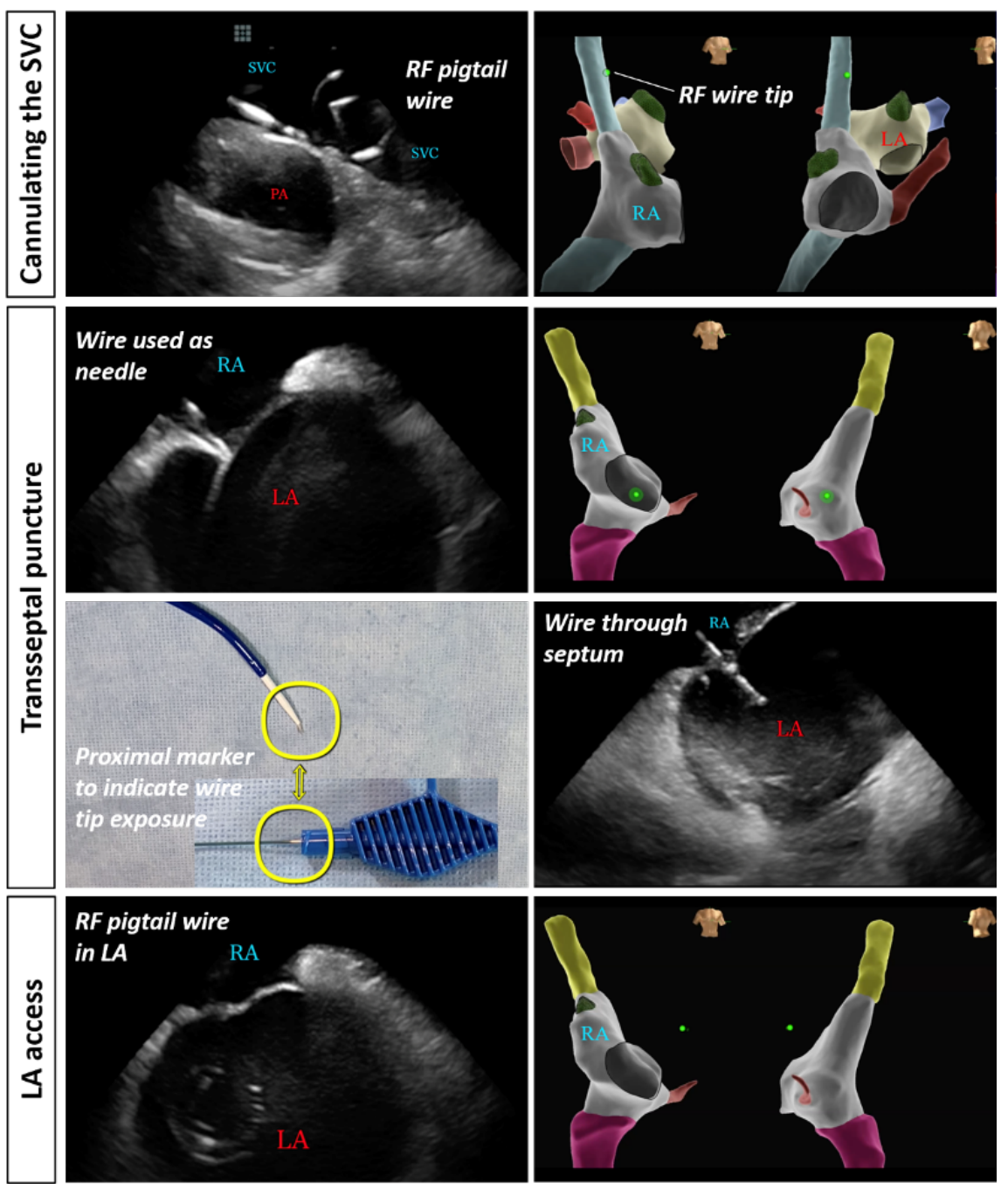

\section{AFS 2021-31}

Abstract Title: Atrioesophageal Fistula and Injury Using Different Energy Sources during Atrial Fibrillation ablation: A Report from the Manufacturer and User Facility Device Experience Database

Author(s): Kun Xiang, MD, PhD

Co-Author(s):

Shreya Ghetiya, MD

Allen Brown, MD

Claude Elayi, MD

Jing $\mathrm{Xu}, \mathrm{PhD}$

Fabrizio Asis, MD

Harikrishna Tandri, MD

John Catanzaro, MD 
UF Health Cardiology

1505 SW Archer Road Gainesville Florida 32606

Introduction | Objectives: Atrioesophageal fistula (AEF) remains a rare and often fatal complication of percutaneous catheter ablation of atrial fibrillation (AF). Ablation energies include radiofrequency (RFE) cryotherapy (CTE) and laser (LE). Although each energy modality has a different mechanism of injury, the reports and association of energy source on the risk of atrioesophageal injury and fistula formation after catheter ablation has not been explored.

Methods: We searched the Manufacturer and User Facility Device Experience database (MAUDE) for adverse events related to AF and atrial flutter ablation procedures from 08/01/2009 to 08/31/2019 was performed. All reports were manually reviewed and e vents associated with AEF formation and esophageal injury were included. Frequency of AEF reporting were compared based on energy used with a Fisher exact test.

Results: Among 1274 reported device adverse events, we identified a total of $5.7 \%$ [73/1274] to have esophageal damage (fistula formation or injury). Among these, AEF formation occurred in $4.1 \%$ [52/1274] and $1.6 \%[21 / 1274]$ were esophageal injury. Among reports with AEF, $71.2 \%$ (37/52) occurred with radiofrequency energy (RF), 26.9\% [14/52] using cryothermal (CTE) and 1.9\% [1/52] with laser energy (LE). There was no statistical difference amongst energy sources for esophageal damage related death or injury $(\mathrm{p}=0.144)$.

Conclusions: The MAUDE database demonstrated that AEF and esophageal has been reported in RFE, CTE and LE ablation. There was no significant difference for the reporting of esophageal fistulas after atrial fibrillation ablation amongst different energy ablation sources. LE is a newer energy modality and continued vigilance is recommended especially when using newer energy sources during ablation for AF. Further research into the precise mechanism of AEF formation and technological advances in esophageal protection technology is warranted to avoid such a devastating major complication.

\section{AFS 2021-32}

Abstract Title: The Association Between Galectin-3 and Cardiac Structure and Comorbidities Among Patients Undergoing Atrial Fibrillation Ablation

Author(s): Evan Hiner, MD

\section{Co-Author(s):}

Daniel Friedman, MD

Kumar Sanam, MD

Sascha Goonewardena, MD

Dipak Shah, MD

Ascension Providence Hospital and Medical Center

16001 W Nine Mile Rd, Southfield, MI 48075

Introduction | Objectives: Galectin-3 (Gal3) is a biomarker of fibrosis and has been associated with more severe forms of AF. The relationship between Gal3 and cardiac structure and function and baseline comorbidities remains poorly characterized.

\section{Methods:}

Sixty-five patients undergoing AF ablation were recruited for this prospective study. Baseline characteristics, including AF history, and echocardiographic parameters were gathered. Biomarkers including, Gal3, IL-6, TNF, IL-10, CRP, and sCD14 levels were drawn pre-procedure. Gal3 levels were divided into tertiles for 
univariate analysis and compared, $1^{\text {st }}$ and $2^{\text {nd }}$ tertiles versus the $3^{\text {rd }}$ tertile. A multivariate analysis was performed using logistic regression.

Results: Significant differences in univariate analysis between the 1st and 2nd tertiles as compared with the third were noted in age (57.1 vs $65.0 \mathrm{p}=0.0065)$, presence of a pacemaker ( $0 \%$ vs $14 \% \mathrm{p}=0.04)$, coronary artery disease $(32 \%$ vs $7 \% \mathrm{p}=0.02)$, TIA ( $14 \%$ vs $0 \% \mathrm{p}=0.04)$, CHF $(18 \%$ vs $2 \% \mathrm{p}=0.04)$, HTN ( $86 \%$ vs $56 \%$ $\mathrm{p}=0.01)$, renal disease $(14 \%$ vs $0 \% \mathrm{p}=0.04), \mathrm{ACEi} / \mathrm{ARB}$ use $(59 \%$ vs $33 \% \mathrm{p}=0.04)$, and diuretic use $(32 \%$ vs $7 \% \mathrm{p}=0.02)$. There were no differences in left atrial size, left ventricular hypertrophy, ejection fraction, type of AF (paroxysmal versus persistent), prior ablation, diabetes, sleep apnea, mitral regurgitation, beta blocker use, amiodarone use, or statin use. Gal3 (12.5 vs $21.7 \mathrm{Ng} / \mathrm{ml} \mathrm{p}=0.0002)$ and sCD14 (4665vs 8154Ng/ml $\mathrm{p}=0.0065$ ) levels were significantly greater in patients in the 3rd Gal3 tertile; there were no differences in CRP, TNF, IL-10, and IL-6. Multivariate logistic regression analysis demonstrated CAD, previous pacemaker, use of diuretics, and prior TIA were more common in the 3rd Gal3 tertile

Conclusions: Gal3 is independently associated with several baseline characteristics but not with cardiac structure/function, AF subtype, or inflammatory biomarkers. Our data suggest that Gal3 may provide unique information regarding myocardial tissue characteristics that cannot be inferred based on standard clinical patient assessment. Further research is required to determine if pre-procedure knowledge of Gal3 can be used to improve prognostication and guide clinical decision making.

\section{AFS 2021-32 Uploaded File(s)}

Patient characteristics stratified by galectin values (3rd vs. 1st/2nd tertile).
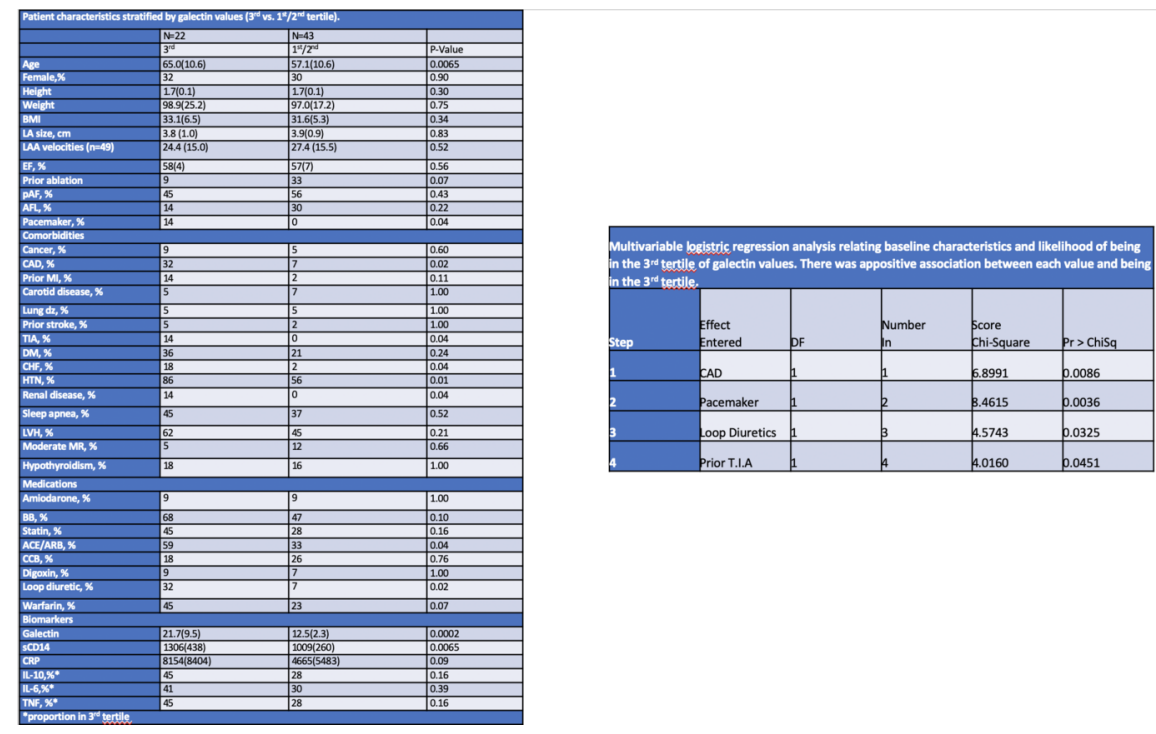

AFS 2021-33

Abstract Title: Impact of atrial fibrillation between transcatheter aortic valve implantation and surgical aortic valve replacement

Author(s): Hyung Ki Jeong, M.D.

Co-Author(s):

Namsik Yoon, MD

Min Chul Kim, MD

Ki Hong Lee, MD 
Hyun Ju Yoon, MD

Kye Hun Kim, MD

Hyung wook Park, MD

Ju Han Kim, MD

Youngkeun Ahn, MD

Myung Ho Jeong, MD

Jeong Gwan Cho, MD

Chonnam National University Hospital

Department of Cardiovascular Medicine, Chonnam National University Hospital, 42 Jaebongro, Dong-gu, Gwangju, 61469, South Korea

Introduction | Objectives: There have been evidences that atrial fibrillation (AF) altered outcomes after transcatheter aortic valve implantation (TAVI) and surgical aortic valve replacement (SAVR) in severe aortic stenosis (AS). However, there was little studies that compared the clinical outcome directly between TAVI and SAVR according to AF. We compared the incidence of AF in aortic valve replacement according to the method of management and how AF would impact their outcomes

Methods: A total of 221, severe AS patients were enrolled from January 2016 to December 2019. Among them, 100 patients were undergone TAVI and 121 were undergone SAVR. We investigated the incidence of $\mathrm{AF}$ and outcomes according to the AF occurrence before and after propensity score (PS) matching for 1-year. The clinical outcomes were defined as death, stroke, admission due to heart failure, and implantation of a permanent pacemaker. The net clinical outcome was composed of death, stroke, and heart failure

Results: The baseline characteristics were different before PS matching. However, it was similar after PS matching. As well as post-procedural or post-operative $\mathrm{AF}(23 \%$ vs. $55.4 \%, P=0.001)$, the new onset $\mathrm{AF}$ incidence was significantly higher in the SAVR group before PS matching $(6.0 \%$ vs $40.5 \%, P<0.001)$ and after PS matching $(7.5 \%$ vs $35.6 \%, P=0.001)$. Post-operative AF had worse impact for heart failure and net clinical outcome in the SAVR group $(\log \operatorname{rank} P=0.049, P=0.033)$. Post-procedural AF in TAVI group also had worse impact for admission due to heart failure $(\log \operatorname{rank} P=0.010)$. TAVI and SAVR did not show significant difference in net clinical outcomes for 1 year follow-up $(\log$ rank $P=0.763)$.

Conclusions: AF had worse impact for both TAVI and SAVR after PS matching. AF occurrence was significantly higher in SAVR group. Therefore, TAVI might be favorable option for severe AS patients who had high risk for AF development or previous AF.

\section{AFS 2021-33}

Uploaded File(s)

AF occurrence before and after procedure \& surgery 


\begin{tabular}{lcccccc}
\hline & \multicolumn{2}{c}{ Before Propensity score matching } & \multicolumn{3}{c}{ After Propensity score matching } \\
& TAVI (N=100) & SAVR (N=121) & P value & TAVI (N=53) & SAVR (N=53) & P value \\
\hline Past History AF (\%) & $24(24.0)$ & $25(20.7)$ & 0.552 & $10(18.9)$ & $16(30.2)$ & 0.176 \\
Paroxysmal & $12(12.0)$ & $16(13.2)$ & 0.322 & $4(7.5)$ & $10(18.9)$ & 0.150 \\
Persistent & $12(12.0)$ & $9(7.4)$ & 0.322 & $6(11.3)$ & $6(11.3)$ & 1.000 \\
Post-procedural AF (\%) & $23(23.0)$ & $67(55.4)$ & 0.001 & $12(22.6)$ & $32(60.4)$ & $<0.001$ \\
New-onset & $6(6.0)$ & $49(40.5)$ & -0.001 & $4(7.5)$ & $19(35)$. & 0.001 \\
$\quad$ Termination < 1 month & 3 & 45 & 0.022 & 3 & 17 & 0.001 \\
$\quad$ Termination 1 3 months & 2 & 0 & 0.010 & 1 & 0 & 1.000 \\
$\quad$ Persistancec > 3months & 1 & 4 & 0.452 & 0 & 2 & 0.495 \\
Pre-Paroxysmal & $5(5.0)$ & $10(8.3)$ & 0.337 & $2(3.8)$ & $8(15.1)$ & 0.093 \\
Pre-Persistent & $12(12.0)$ & $7(5.8)$ & 0.101 & $6(11.3)$ & $5(9.4)$ & 0.750 \\
Rhythm control & & & & & & \\
$\quad$ Amiodarone & $9(9.0)$ & $16(14.9)$ & 0.219 & $4(7.5)$ & $9(17.0)$ & 0.236 \\
Ic & 0 & 0 & - & 0 & 0 & - \\
$\quad$ DC cardioversion & $1(1.0)$ & $7(5.8)$ & 0.075 & $1(1.9)$ & $6(11.3)$ & 0.051 \\
\hline AF-atrial fibrillation & & & & & &
\end{tabular}

AFS 2021-34

Abstract Title: Atrial fibrillation burden, episode duration and frequency in relation to quality-of-life in patients with implantable cardiac monitor

Author(s): Victoria Jansson, MD, PhD student

Co-Author(s):

Lennart Bergfeldt, Professor

Jonas Schwieler, Associate professor

Göran Kennebäck, Associate Professor

Aigars Rubulis, MD PhD

Steen Jensen, Associate Professor

Pekka Raatikainen, Professor

Elena Sciaraffia, MD PhD

Carina Blomström Lindqvist, Professor

Department of Medical Sciences

Akademiska sjukhuset, ing 35, 2nd floor, 75185 UPPSALA, SWEDEN

\section{Introduction | Objectives:}

We assessed the relationship between atrial fibrillation (AF) characteristics and health-related quality of life (QoL), and which AF characteristic had the greatest impact.

Methods: The AF characteristics burden (percentage of time in AF), duration, and number of AF episodes/month were obtained from implantable cardiac monitors during the 2-month run-in period in 150 patients included in the randomized CAPTAF (Catheter Ablation compared with Pharmacological Therapy for Atrial Fibrillation) trial comparing early ablation and antiarrhythmic drug therapy. QoL was measured by the General Health and Vitality dimensions of the 36-Item Short-Form Health Survey. AF characteristics were analyzed continuously and in quartiles (Q1-Q4).

\section{Results:}

Greater AF burden ( $\mathrm{p}=0.003)$ and longer AF episodes $(\mathrm{p}=0.013)$ were associated with impaired QoL (Vitality score only) in simple linear regression analyses. Greater AF burden was, however, the only AF characteristic associated with lower QoL, when adjusted for sex, type of AF, hypertension, heart rate > $110 \mathrm{bpm}$ during $\mathrm{AF}$, and beta-blocker use in multiple linear regression analyses. For every $10 \%$ increase in AF burden, there was a 1.34 point decrease of Vitality score (95\% confidence interval (CI) 
$-2.67-0.02, \mathrm{p}=0.047)$. The Vitality score was 12 points lower (95\% CI $-22.73--1.27, \mathrm{p}=0.03)$ in patients with an AF burden $>33 \%$ (Q4) versus those with $<0.45 \%$ (Q1), but only in unadjusted analysis.

\section{Conclusions:}

AF burden had a greater impact on QoL (Vitality), than the duration and number of AF episodes, corroborating that $\mathrm{AF}$ burden may be the preferred outcome measure of rhythm control in trials including relatively healthy AF populations.

AFS 2021-34

Uploaded File(s)

Table. Atrial fibrillation burden, episode duration and frequency as continuous variables associated with health-related quality of life in adjusted and unadjusted models.

Atrialfibrillationburdenepisode.xlsx

\begin{tabular}{ll}
\hline & Simple regression analyses \\
\hline & $(\mathbf{1 4 1}$ patients $)$ \\
& General Health \\
& Estimate \\
& $(\mathbf{9 5 \%} \mathbf{C I})$ \\
& -0.65 \\
& $(-1.54-0.26)$ \\
AF burden, $[\%]^{\mathbf{b}}$ & -0.04 \\
& $(-0.17-0.09)$ \\
AF episode duration, $[\mathbf{m i n}]^{\mathbf{c}}$ & 0.01 \\
& $(-0.02-0.04)$ \\
AF frequency, $[$ episodes $/$ month $]$ & AF $=$ Atrial fibrillation; AF burden $=\%$ of time spent in AF; AF episode duration \\
& $\mathrm{a}=$ models adjusted for sex, heart rate $>110$ bpm during AF, hypertension, type of \\
& $\mathrm{b}=$ with 10\% increase; \\
& $\mathrm{c}=$ with 1-hour increase and 122 patients included in simple regression analyses anc \\
&
\end{tabular}

\section{AFS 2021-35}

Abstract Title: Comparison of Traditional Versus High Power Short Duration Radiofrequency Ablation on Esophageal Temperature

Author(s): Cameron Whitler, DO

Co-Author(s):

Amir Naqvi, DO

Evan Hiner, MD

Dipak Shah, MD

Ascension Providence Hospital/MSU-CHM

16001 W. 9 Mile Road 4th Flr Fisher Building Southfield, MI 48075

Introduction | Objectives: Atrial fibrillation (AF) ablation, while generally safe, can result in esophageal injury. Esophageal temperature monitoring is routinely used to guide radiofrequency ablation (RFA). High power short duration (HPSD) RFA is becoming more utilized. Traditional RFA relies on distal conductive heating while HPSD creates lesions by local conductive and resistive heating; thereby, potentially minimizing 
esophageal injury. The objective of this study is to compare traditional versus HPSD RFA on esophageal temperature during AF ablation.

Methods: We conducted a retrospective analysis of all consecutive AF ablations performed over an 18month period. All ablations were performed by one operator who changed his practice from traditional to HPSD RFA. The parameters of the traditional ablation were 15-30 grams of contact force, force time integral (FTI) of 400-600, and power of 20-30 Watts. The parameters for HPSD were contact force of 5-20 grams with 50 Watts of power applied for 3-15 seconds targeting a $10 \Omega$ impedance drop. Data analysis included type of method used, temperature changes and basic characteristics including underlying type of AF. Patients were excluded if they had prior left atrial ablation. A simple t-test analysis was done comparing both the max temperatures, as well as the absolute temperature change for each group.

Results: A total of 145 patients were included in the analysis. 72 patients in the traditional ablation group had a mean max temperature of $37.6+1.39$ @C. Comparatively, the 73 patients in the HPSD group had a mean max temperature of $37.3+0.646 @ \mathrm{C}$, which was trending towards, but did not achieve statistical significance $(\mathrm{p}=0.099)$. However, when comparing the absolute temperature change, HPSD RFA demonstrated a significantly less increase in temperature than the traditional RFA $(1.14+0.48 @ \mathrm{C}$ vs. 1.69 $+0.60 @ \mathrm{C}(\mathrm{p}<0.0001))$.

Conclusions: HPSD RFA resulted in a significantly lower esophageal temperature change likely due to less distal conductive heating. Studies suggest numerically less atrioesophageal fistula (AEF) with HPSD, albeit it the numbers are small. With an increasing prevalence of HPSD RFA being used significantly less AEF may be seen in $\mathrm{AF}$ ablation registries.

\section{AFS 2021-36}

Abstract Title: Source agnostic Artificial Intelligence analysis of Biotronik Biomonitor 3 ECG data.

Author(s): Pierre Jais, MD

\section{Co-Author(s):}

Philip Currie, MBBS - Cardiovascular Services

David Playford, MBBS FRACP PhD FCSANZ FESC FACCC - Professor, The University of Notre Dame Australia

Luke Bollam, BEng - Alerte Digital Health

Razali Mohamad, MEng, BCM - Alerte Digital Health

Kushwin Rajamani, MBBS

Rukshen Weerasooriya, MBBS

universite de Bordeaux

Place de la Victoire, 33000 Bordeaux, France

Introduction | Objectives: Implanted loop recorders (ILR) have an important role to play in the detection of atrial fibrillation (AF). Continuous home monitoring of data from ILR may result in false positive AF detections due to a number of factors including noisy signal and frequent ectopic beats. A pilot study was designed to evaluate the use of AI to improve AF detection from Biotronik Biomonitor 3 (BM3) recordings using a source agnostic AI strategy.

Methods: A total of 1015 individual BM3 recordings were used in this study. The BM3 data was uploaded to an ECG AI web application and was then manually labelled by cardiologists. An AI model was created based upon labelled data from a clinical database of 18,000 beat and rhythm labelled 12-lead ECGs representing a wide range of arrhythmias and beat disorders. For the purpose of this pilot study, the following minimal arrhythmia labelling criteria were pre-defined: High relevance (eg atrial fibrillation), Low 
relevance (eg sinus rhythm plus ectopics), Not appraisable (eg noise). The following experiments (Exp) were conducted: Exp 1. An AI model was created according to the minimal labelling criteria only using 12-lead ECG data; Exp 2. The existing AI model was assessed on BM3 data with no training using BM3 data; Exp 3. The existing AI model was fine tuned using BM3 data under the following 2 scenarios: Exp 3a. $29 \%$ of BM3 data (295 recordings) used for fine tuning and evaluated using $71 \%$ of BM3 data (717 recordings). Exp 3b $68 \%$ of BM3 data (688 recordings) used for fine tuning and evaluated using $32 \%$ of BM3 data (327 recordings).

Results: Exp 1 AUC 0.975004

\begin{tabular}{llllll}
\hline HIGH RELEVANCE & HIGH RELEVANCE & LOW RELEVANCE & LOW RELEVANCE & NOT APPRAISABLE & NO \\
\hline Se & $\mathrm{Sp}$ & $\mathrm{Se}$ & $\mathrm{Sp}$ & Total & Perc \\
$95.85 \%$ & $91.59 \%$ & $91.59 \%$ & $95.85 \%$ & 22 & 1.02 \\
\hline
\end{tabular}

Exp 2 AUC 0.8209

\begin{tabular}{llllll}
\hline HIGH RELEVANCE & HIGH RELEVANCE & LOW RELEVANCE & LOW RELEVANCE & NOT APPRAISABLE & NO \\
\hline Se & $\mathrm{Sp}$ & $\mathrm{Se}$ & $\mathrm{Sp}$ & Total & Perc \\
$95.13 \%$ & $75.08 \%$ & $75.71 \%$ & $95.19 \%$ & 19 & 1.87 \\
\hline
\end{tabular}

Exp 3a AUC 0.931319

\begin{tabular}{llllll}
\hline HIGH RELEVANCE & HIGH RELEVANCE & LOW RELEVANCE & LOW RELEVANCE & NOT APPRAISABLE & NO \\
\hline $\mathrm{Se}$ & $\mathrm{Sp}$ & $\mathrm{Se}$ & $\mathrm{Sp}$ & Total & Perc \\
$95.90 \%$ & $97.03 \%$ & $97.01 \%$ & $95.19 \%$ & 12 & 1.65 \\
\hline
\end{tabular}

Exp 3b AUC 0.960144

\begin{tabular}{llllll}
\hline HIGH RELEVANCE & HIGH RELEVANCE & LOW RELEVANCE & LOW RELEVANCE & NOT APPRAISABLE & NO \\
\hline $\mathrm{Se}$ & $\mathrm{Sp}$ & $\mathrm{Se}$ & $\mathrm{Sp}$ & Total & Perc \\
$95.16 \%$ & $98.99 \%$ & $98.98 \%$ & $93.65 \%$ & 5 & 1.53 \\
\hline
\end{tabular}

Conclusions: An existing AI model based on 12 lead ECG data is generalisable to a new form of ECG data from the BM3. Furthermore, fine tuning using the new BM3 data resulted in improved sensitivity and specificity for AF detection.

\section{AFS 2021-37}

Abstract Title: Outcomes of radiofrequency catheter ablation of atrial fibrillation: the experience of a referral center in Lebanon

Author(s): Marwan Refaat, MD

Co-Author(s):

Maysam Al-Housari, MD

Hussein Khachfe, BS 
Hamza Salhab, BS

Maurice Khoury, MD

Bernard Abi-Saleh, MD

American University of Beirut Medical Center

Cairo Street, Beirut, Lebanon

Introduction | Objectives: Atrial fibrillation (AF) and atrial flutter ablation are increasingly used in clinical practice. We aimed to study the outcomes of ablated AF and atrial flutters at a major tertiary referral medical center in Lebanon.

Methods: We followed the last 99 patients after pulmonary vein isolation (PVI) and flutters ablations performed at the American University of Beirut Medical Center utilizing the circumferential mapping catheter technique, Intracardiac Echography (ICE) and a 3D electroanatomic mapping system. Typical flutters were ablated at the cavotricuspid isthmus under ICE guidance, perimitral flutters were ablated by a left anteroseptal line and the rest of the flutters had an activation map and/or an entrainement map then ablated accordingly. All patients had failed at least one antiarrhythymic drug (AAD). We documented recurrence of the arrhythmia on and off AADs.

Results: Our patient population included 32\% paroxysmal AF, $46 \%$ persistent AF and $22 \%$ long standing persistent $\mathrm{AF}$. In the first six months after ablation, $92 \%$ maintained sinus rhythm on and off AAD. Over a longer follow-up (12 months), 83\% maintained sinus rhythm. Off all antiarrhythmic drugs $77 \%$ had no recurrence of the arrhythmia at six month follow up and $73 \%$ maintained sinus rhythm without recurrence of the arrhythmia at 12 months follow-up (Figure). Only five patients underwent repeat ablations and this revealed conduction recovery in at least 1 of the previously isolated PVs. The rest of the recurred arrhythmias were either rate controlled or the patient decided to accept a low burden of AF on an AAD. The procedure-related complication rate was very low; one patient had a pseudoaneurysm requiring surgical repair and one patient had a pericardial effusion that required drainage. None had pulmonary vein stenosis.

Conclusions: Pulmonary vein isolation and atrial flutters ablation are safe and efficacious for the maintenance of sinus rhythm in this Lebanese cohort of patients with drug-resistant arrhythmias. They obviate the need for antiarrhythmic drugs in a substantial proportion of Lebanese patients.

AFS 2021-37

\section{Uploaded File(s)}

Follow up for 1 year of 99 patients post atrial fibrillation ablation. 

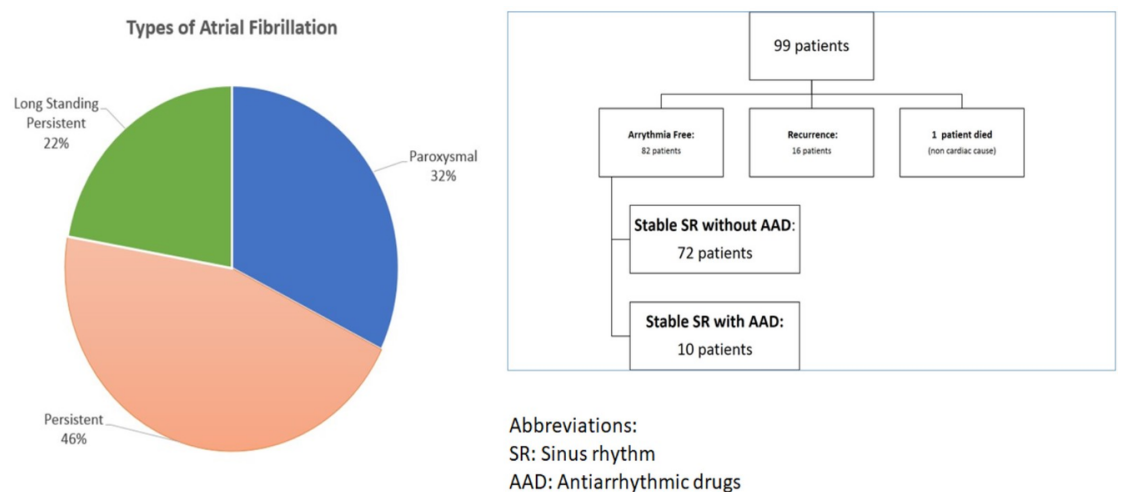

\section{AFS 2021-38}

Abstract Title: Non-contact mapping of organised atrial rhythms - early "real-world" experience

Author(s): Michael Pope, BM BSc MRCP

\section{Co-Author(s):}

Timothy Betts, MD FRCP - Oxford University Hospitals NHS Foundation Trust

Oxford University Hospitals NHS Foundation Trust

John Radcliffe Hospital Headley Way

Introduction | Objectives: "Supermap" is a novel algorithm for the mapping of organised atrial rhythms using non-contact charge density mapping (Acutus Medical).We report our early experience.

Methods: Patients enrolled in parallel research studies using the AcQMap system were included. Mapping of both atrial tachycardias (AT) and paced rhythms (PR) was performed using the Supermap non-contact algorithm and, prior to analysis, was repeated with contact mapping using Ensite Precision (Abbott Medical). AT mechanism or linear block (in the case of PR) was confirmed using a combination of mapping results, conventional electrophysiological manoeuvres, and the response to ablation. The time taken for map creation, point density and accuracy were compared between the 2 systems.

Results: Ten patients (age 64(IQR10), 40\% male) were included with 26 maps obtained (20 ATs, 6 PRs; 17 left atrium). Six had previous ablation, 2 cardiac surgery. Parallel contact maps were obtained in 22 (12 using circular mapping catheters, 4 using HD grid, 6 using ablation catheter). Supermap was accurate in $92 \%$ and the contact map in $82 \%$ ( $\mathrm{p}=0.3923$ ). Mapping was faster using Supermap (4min and 32s (IQR $1 \mathrm{~min} 42 \mathrm{~s})$ vs $8 \min 21 \mathrm{~s}($ IQR $4 \min 20 \mathrm{~s}), \mathrm{p}<0.0005)$ and achieved a higher point density (5222 (IQR6161) vs 953 (IQR2777), $\mathrm{p}=0.0014$ ).

Conclusions: Supermap is a fast and accurate tool for mapping of both atrial tachycardias and paced rhythms. 


\begin{abstract}
AFS 2021-38
Uploaded File(s)

Figure 1. Mapping duration was faster using the Supermap non-contact algorithm (panel A) and obtained a higher number of EGM points (panel B).
\end{abstract}
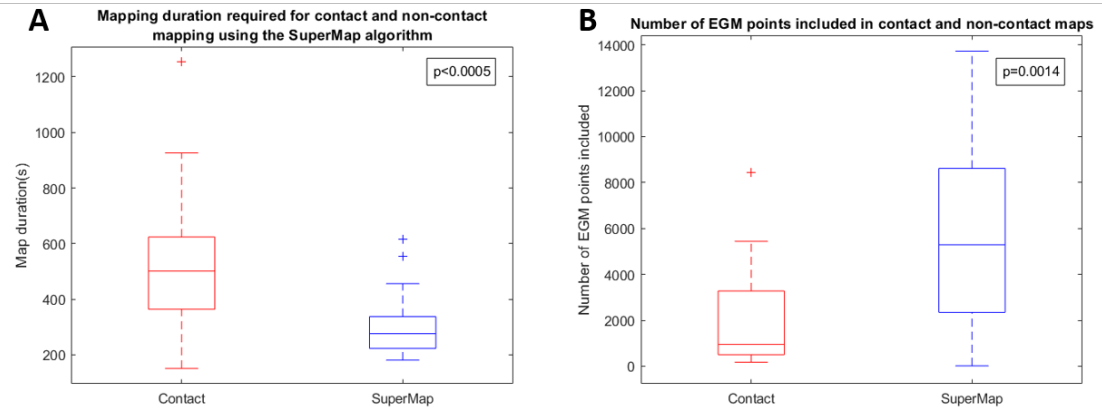

AFS 2021-39

Abstract Title: Procedural Effects of Active Esophageal Warming During Pulmonary Vein Isolation Cryoablation for Atrial Fibrillation

Author(s): Zachary Hollis, MD

Co-Author(s):

Daniel Alexander, DO - Winchester Medical Center

Rebekah Smith, RN - Winchester Medical Center

Erik Kulstad, MD, MS - UT Southwestern Medical Center

Winchester Medical Center

Winchester Medical Center, 1840 Amherst Street, Winchester, VA 22601

Introduction | Objectives: Electrical isolation of the pulmonary veins utilizing the Cryoablation balloon (Arctic Front, Medtronic) has been established as an effective treatment for both paroxysmal and persistent atrial fibrillation (AF). Esophageal injury as a result of convective thermal transfer is a rare, but potentially fatal complication of both radiofrequency and cryoablation techniques. Active warming of the esophagus during cryotherapy offers a proactive approach to countering potential cryothermal injury which does not require repositioning during use. This prospective study (NCT04087122) measured the difference in procedure time between patients treated with and without an esophageal warming device while undergoing cryoablation for the treatment of AF.

Methods: Patients scheduled for cryoablation were consented for enrollment, with cryoablation performed in standard fashion with the exception of the placement of an active esophageal warming device in place of the standard temperature sensor. Warming device temperature was set to $42^{\circ} \mathrm{C}$. Procedure times, left atrial time, and fluoroscopy usage for each case were then compared to the previous consecutive cryoablation control patients.

Results: A total of 20 patients were prospectively enrolled and received active esophageal warming in place of standard LET monitoring. Mean total procedure time was 126 minutes (95\% CI 112 to 139 minutes) and median time was 119.5 minutes (IQR 29 minutes). A total of 25 patients served as historical controls, with mean total procedure time of 141 minutes (95\% CI 126 to 156 minutes), and median time of 132 minutes (IQR 28 minutes), $\mathrm{p}=0.03$, Mann-Whitney U Test. Time from transeptal access to last pacing maneuver to 
confirm exit block (used as a surrogate for left atrial time); and the total fluoroscopy time were shorter in the active esophageal warming group by $5 \%$ and $6 \%$ respectively. These trended towards, but did not reach, statistical significance. In one and three month follow up, no complications, including stroke or esophageal injury were identified.

Conclusions: Use of active esophageal warming avoids the need for procedural interruptions and temperature probe repositioning, which appears to improve efficiency by reducing total procedure time.

\section{AFS 2021-40}

Abstract Title: Esophageal cooling for protection during AF ablation: effects on radiofrequency lesion formation in the left atrium.

Author(s): Lisa W. Leung, MBChB (Hons), MRCP

Co-Author(s):

ahmed ElBatran, MD

Abhay Bajpai, MD - St. George's NHS Trust

Zia Zuberi, PhD - St. George's NHS Trust

Anthony Li, MD - St. George's NHS Trust

Mark Norman, PhD

Riyaz Kaba, MD - St. George's NHS Trust

Manav Sohal, MD - St. George's NHS Trust

Zaki Akhtar, MRCP

Banu Evranos, MD

Hanney Gonna, MRCP

Idris Harding, $\mathrm{PhD}$

Nawaf Al Subaie, PhD

Jamal Hayat, MD - St. George's NHS Trust

John Louis-Auguste, MD - St. George's NHS Trust

Mark Gallagher, MD - St. George's NHS Trust

SGUL

Blackshaw Road, London, SW170QT

Introduction | Objectives: The IMPACT study established the role of controlled esophageal cooling in preventing thermal injury during radiofrequency (RF) ablation for atrial fibrillation (AF) guided by Ablation Index (AI). The effect of esophageal cooling on ablation delivery and acute outcomes had not been previously studied. Objective: To determine the effect of esophageal cooling on the formation of RF lesions, the ability to achieve procedural endpoints and the characteristics of RF lesions associated with esophageal injury.

Methods: AF ablation was guided by AI (30W at 350-400 AI posteriorly, $40 \mathrm{~W}$ at 450 AI anteriorly). A blinded 1:1 randomization assigned patients to the use of ensoETMß device to keep esophageal temperature at 4 degrees during ablation or standard practice using a single-sensor temperature probe. Ablation parameters and short-term outcomes were analysed.

Results: We recruited 188 patients. Thermal injury to the mucosa was significantly more common in the control group than in those receiving esophageal protection $(12 / 60$ versus $2 / 60 ; \mathrm{P}=0.008)$. Procedure and 
fluoroscopy times were similar. First pass pulmonary vein isolation and reconnection at the end of the waiting period were similar in both randomized groups $(51 / 64$ vs $51 / 68 ; \mathrm{p}=0.54$ and $5 / 64$ vs $7 / 68 ; \mathrm{p}=0.76$, respectively). Posterior wall isolation was also similar: $24 / 33$ vs $27 / 38 ; \mathrm{p}=0.88$. Ablation effect on tissue, measured in impedance drop, was also similar: $8.6 \Omega$ (IQR: 6-11.8) vs $8.76 \Omega$ (IQR: 6-12.2; $\mathrm{p}=0.25$ ). Arrhythmia recurrence was similar at 6 months and 12 months $(\mathrm{p}>0.05)$.

Conclusions: Esophageal cooling has been shown to be effective in reducing ablation-related thermal injury during RF ablation. AI data show that this protection does not make it any more difficult to achieve standard procedural endpoints or clinical success in the short and mid term.

AFS 2021-40

Uploaded File(s)

Impedance drop of radiofrequency lesions delivered in the left atrium in both randomized groups.
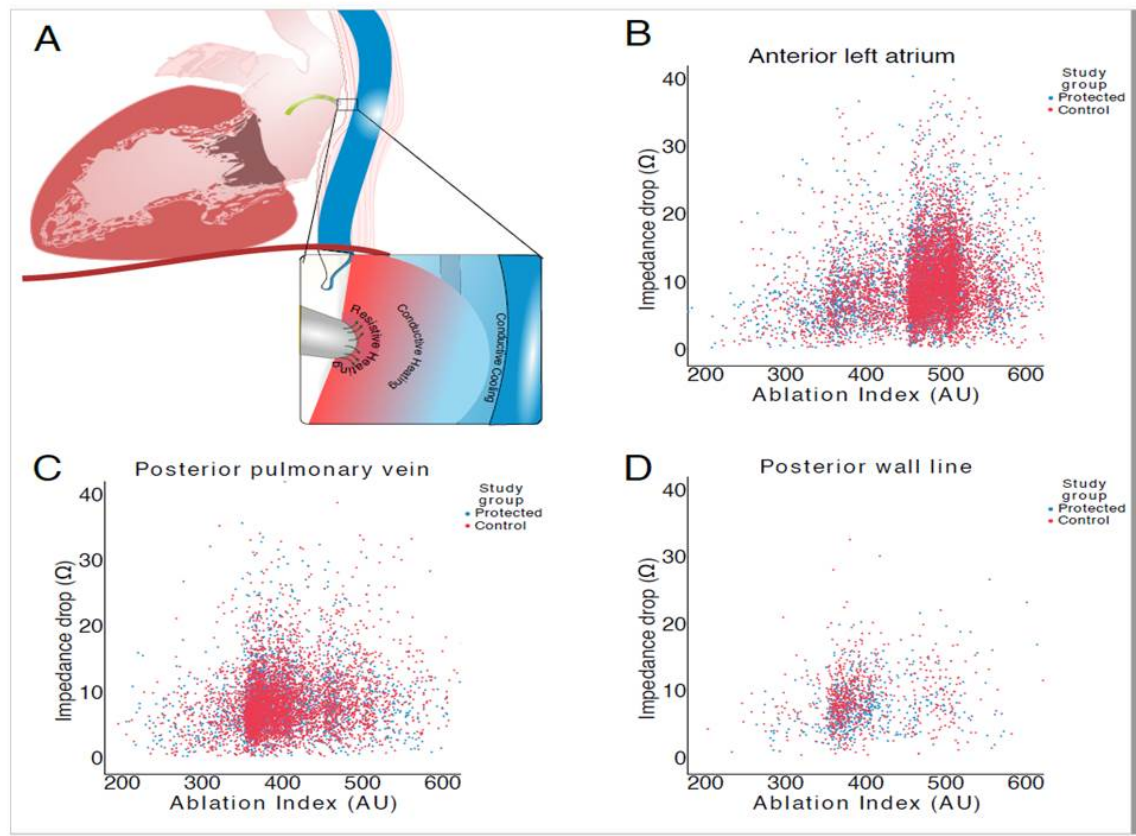

\section{AFS 2021-41}

Abstract Title: Catheter Ablation or Antiarrhythmic Drugs for First-Line Therapy of Atrial Fibrillation: A Meta-analysis of Randomized Controlled Trials

Author(s): Mohit K. Turagam, MD

Co-Author(s):

Daniel Musikantow, MD

William Whang, MD

Jacob Koruth, MD

Marc Miller, MD

Noelle Langan, MD 
Aamir Sofi, MD

Subbarao Choudry, MD

Srinivas Dukkipati, MD

Vivek Reddy, MD

Mount Sinai Hospital

1, Gustav L. Levy Street, New York, NY-10029

Introduction | Objectives: The recently published multicenter EAST-AFNET4 trial showed that as compared with usual care, early rhythm control of atrial fibrillation (AF) with either antiarrhythmic drugs (AADs) or catheter ablation improved cardiovascular outcomes. The optimal therapeutic modality to achieve early rhythm control is unclear. We performed a meta-analysis of all randomized studies (RCTs) comparing the safety and efficacy of AF ablation as first-line therapy when compared with AADs in patients with paroxysmal AF.

Methods: We searched PubMed, Scopus, Google Scholar, and various major scientific conference sessions from January 1, 2000 until to November 23, 2020 for RCTs that had at least 12 months of follow-up and compared clinical outcomes of ablation versus AADs as first-line therapy in adults with paroxysmal AF. Analysis was performed using random-effects model using Mantel-Haenszel method and results presented as $95 \%$ confidence interval.

Results: Six RCTs met inclusion criteria, including 1,212 patients (ablation=609, AADs=603). Age range 50-60 yrs, mean duration of AF $1.3 \pm 2.3$ yrs and mean LA diameter was $40 \pm 6 \mathrm{~mm}$. In RAAFT, RAAFT-2 and MANTRA-PAF , ablation was performed using radiofrequency energy while cryoballoon was employed in the STOP AF , EARLYAF , and CRYO-FIRST clinical trials. The AADs arm primarily employed class IC drugs in $82 \%$ and class III drugs in 14\%. Study follow-up ranged from 12 to 24 months (mean $16 \pm 6$ months). Compared with AADs, catheter ablation reduced recurrent atrial arrhythmia $(32.3 \%$ vs $53 \%$; risk ratio $[\mathrm{RR}] 0.62,95 \%$ CI $0.51-0.74, \mathrm{p}<0.00001)$ with a number needed to treat of 5 . Ablation also reduced symptomatic atrial arrhythmia ( $11.8 \%$ vs $26.4 \%$, RR $0.44,95 \%$ CI, 0.27 to $0.72, \mathrm{p}=0.001)$ and hospitalization ( $5.6 \%$ vs $18.7 \%$, RR $0.32,95 \%$ CI 0.19 to $0.53, \mathrm{p}<0.00001)$ with no significant difference in serious adverse events between the groups ( $4.2 \%$ vs $2.8 \%$, RR $1.52,95 \%$ CI 0.81 to $2.85, \mathrm{p}=0.19$ ). (Figure )

Conclusions: In this meta-analysis of first-line therapy of patients with paroxysmal AF, as compared to antiarrhythmic drugs, catheter ablation as first-line therapy in patients with paroxysmal AF leads to reductions in AF recurrence and hospitalizations, with no difference in major adverse events.

\section{AFS 2021-41}

Uploaded File(s)

Summary Plot of Clinical Outcomes 


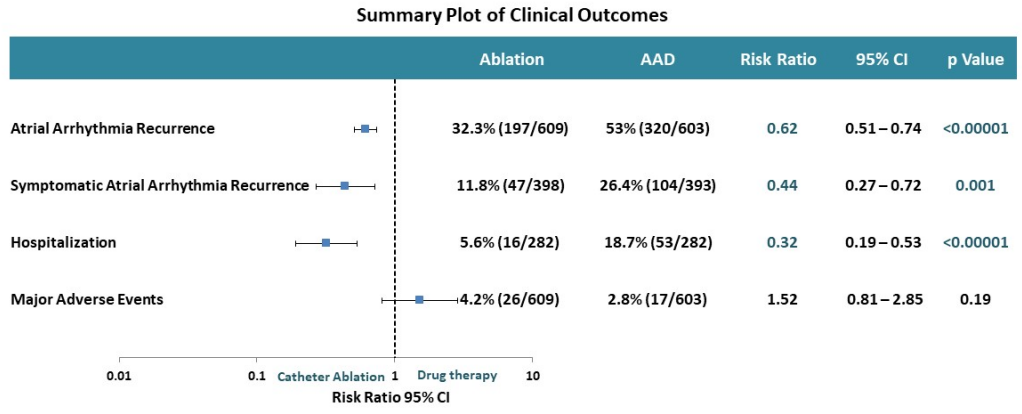

AFS 2021-42

Abstract Title: Electrolytic Effects from a Clinical Endocardial Pulsed Field Ablation System in a Benchtop Model: a Comparison of Gas Formation with Focal RF Ablation

Author(s): Christopher E. Woods, MD/PHD

Co-Author(s):

Raju Viswanathan, PhD - Vice President of Research and Development, Farapulse

Vivek Reddy, MD - Director of Cardiac Arrhythmia Services, Mount Sinai

MPMC/CPMC Sutter Health

1501 Trousdale Drive, Burlingame, CA, 94010

Introduction | Objectives: Endocardial pulsed field ablation (PFA) has electrolytic effects that are echogenic and can be altered via device and delivery parameters. Quantification of PFA-generated ,in comparison with radiofrequency (RF), bubbles can inform PFA's embolic safety profile.

Methods: The PFA system was described previously ${ }^{1}$. A bubble trap described previously was used ${ }^{2}$. Room temperature saline $(4.5 \mathrm{~g} / \mathrm{L} \mathrm{NaCl}$ to mimic clinical impedance) flowing at a rate of $0.8 \mathrm{~L} / \mathrm{min}$ was used. PFA was delivered and quantification of gas burden was made with a bubble counter (Gampt BCC300). Gas emission was qualified via ICE echocardiography (RF and PFA). RF applications were made using a commercial system (Boston Scientific Maestro 4000/Blazer Model 9620). The data was compared with published values from an in-vivo RF model ${ }^{3}$.

Results: 6 scenarios for PFA delivery were investigated (figure 1): free-floating vs in contact with porcine myocardium (tissue), $2.0 \mathrm{vs} 1.8 \mathrm{kV}$ amplitudes and two catheter shapes. A total of 8 applications were delivered per session per clinical studies ${ }^{1}$. Average gas volume and maximal bubble diameter was $1.52 \pm 1.44 \mathrm{~nL}$ and $85 \mathrm{~mm}$, respectively, across all PFA scenarios. RF applications to tissue (excluding steam pops) created an average gas volume (nl) of $177 \pm 134$ or $59.3 \pm 44.7$ when adjusted for flow rate ${ }^{2}$ (factor 0.33 ), similar to previous data. Saline vs tissue PFA values were similar. Median bubble diameter was smaller with PFA than RF (18 vs 30um, respectively). PFA bubbles were visible only on ICE and only during the few seconds of delivery. RF bubbles were visible via ICE, persisting up to one minute after end of delivery.

Conclusions: Total volume and maximal diameter of bubbles produced with a clinical PFA system were lower when compared with RF values. The density of small-sized bubbles occurring in a short PFA delivery 
duration contribute to the echogenicity of PFA delivery. Similar results between PFA deliveries to tissue and saline support electrolytic rather than heat-related bubble formation. References

- Takami et al, Circ. Arrythm. Electrophysiol. 9:e003226, 2016.

- Vivek Reddy et al, Am. Coll. Cardiol . 74(3) (2019 Jul 23) 315 - 326.

- van Es et al, Cardiovasc. Electrophysiol. 30 (2019) $2071-2079$.

\section{AFS 2021-42}

\section{Uploaded File(s)}

Figure 1. Microbubble size distribution, presented as distribution with media and interquartile ranges, and bubble volumes observed with each scenario.

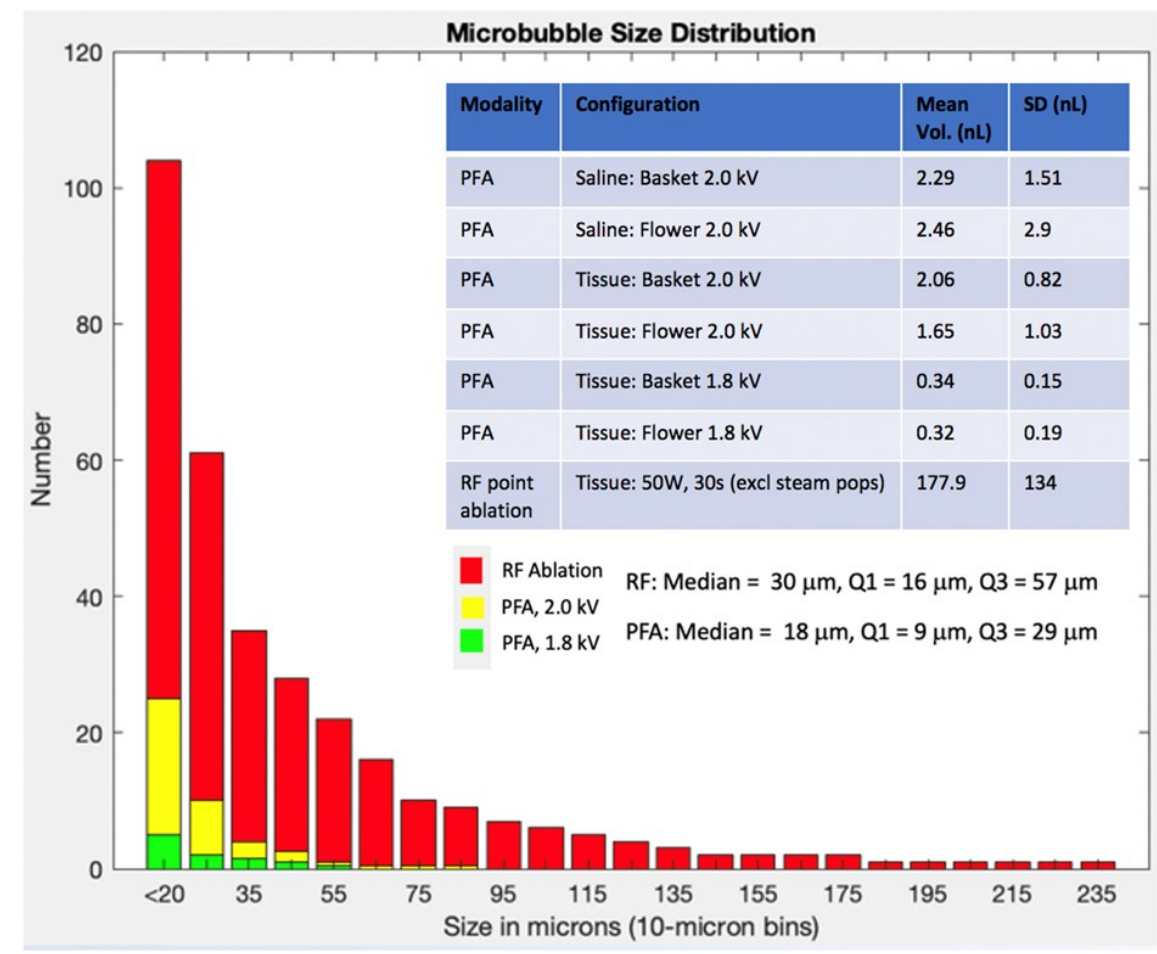

\section{AFS 2021-43}

Abstract Title: A histological study of the atria in patients with isolated mitral regurgitation with and without atrial fibrillation

Author(s): Jayaprakash Shenthar, MD, DM, FACC, FRCP (London)

Co-Author(s):

Saligrama Ramegowda Kalpana, MD

Deepak Padmanabhan, MD, DM

Manish K Rai, MD, DM

Ankit Singh, MD, DM

Bharatraj Banavalikar, MD, DM

Darshan Krishnappa, MD, DM 


\title{
SRI JAYADEVA INSTITUTE OF CARDIOVASCULAR SCIENCES \& RESEARCH
}

\author{
SRI JAYADEVA INSTTUTE OF CARDIOVASCULAR SCIENCES \& RESEARCH BANNERGHATTA \\ MAIN ROAD, PHASE 3 9TH BLOCK, JAYANAGAR, BENGALURU- 560069
}

Introduction | Objectives: Atrial fibrillation and left atrial thrombus are less common in isolated mitral regurgitation than mitral stenosis or mixed mitral valve disease of rheumatic etiology. The histopathologic changes in patients with and without atrial fibrillation in patients with isolated rheumatic mitral regurgitation are unknown. We aimed to discern the differences in histological findings in patients with severe rheumatic mitral regurgitation with and without atrial fibrillation.

Methods: Patients with severe isolated rheumatic mitral regurgitation undergoing valve replacement surgery underwent endocardial biopsy from right atrial appendage (RAA), left atrial appendage (LAA), right atrial free wall $(\mathrm{RAFW})$, left atrial free wall (LAFW), left posterior wall (LPW) and mitral valve. Group I consisted of patients in sinus rhythm and Group II included patients with atrial fibrillation. We analyzed and compared these samples between the two groups for myocyte hypertrophy, interstitial and endocardial fibrosis, inflammatory cells, Aschoff bodies, interstial adipose tissue, cellular vacuolation, and iron/amyloid deposition.

Results: Of the 25 patients, 12 were in Group I and 13 in group II. Myocyte hypertrophy was the most common histological finding and was more prevalent in Group I ( $100 \%$ vs $92.3 \%, \mathrm{p}=0.33)$. More severe myocyte hypertrophy was seen in Group I ( $60 \%$ vs $18 \%$ showing severe hypertrophy, $\mathrm{p}=0.04$ ) and in right atrium $(22.7 \%$ vs $11.4 \%, \mathrm{p}=0.059)$. Interstitial fibrosis was another common finding with near even distribution at all sites and without significant difference between the two groups. Prevalence and severity of vacuolar degeneration was higher in Group II ( $91 \%$ vs $60 \%$ showing severe changes, $\mathrm{p}=0.09$ ). Interstitial adipose tissue deposition was more common in Group I ( $30 \%$ vs $25 \%, \mathrm{p}=0.06$ ).

Conclusions: Patients with severe rheumatic mitral regurgitation and atrial fibrillation have more vacuolar degeneration whereas myocyte hypertrophy and interstitial adipose tissue deposition are more common in sinus rhythm.

\section{AFS 2021-43 Uploaded File(s)}

Photomicrograph showing A) Interstitial fibrosis 40X Haematoxylin \& Eosin stain, B) Myocyte vacuolar degeneration 40X Haematoxylin \& Eosin stain 


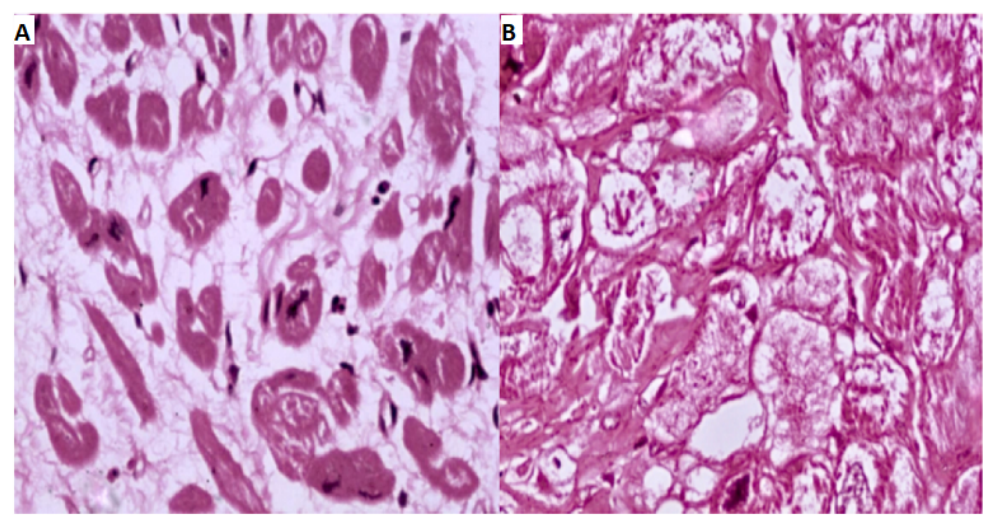

AFS 2021-44

Abstract Title: Body surface mapping of P-waves in sinus rhythm to predict recurrent following cardioversion for atrial fibrillation

Author(s): Ibrahim Antoun, MD

Co-Author(s):

Chu Gavin, MBChB, PhD

$\mathrm{Xin} \mathrm{Li}, \mathrm{PhD}$

Zakariyya Vali, MBChB, MRCP

Akash Mavilakandy, MBChB

Tiago Paggi De Almeida, PhD

Andre Ng, MBChB, MRCP, PhD - Professor in electrophysiology, University of Leicester

University of Leicester

University road, Leicester, UK

Introduction | Objectives: Clinical classification (paroxysmal vs. persistent) for atrial fibrillation (AF) remains inadequate for informing substrate underlying arrhythmogenesis and recurrence. This study was performed to examine $\mathrm{P}$ waves during sinus rhythm (SR) following DC cardioversion (DCCV) using highdensity body surface mapping (BSM) to correlate with DCCV outcome 12 months to provide insight on possible underlying substrate.

Methods: 56 patients who underwent DCCV for AF were recruited. P-wave duration corrected by heart rate (PWDc), P-wave amplitude (PWA) and P-wave temporal stability (TS) were measured using 128-lead BSM immediately following DCCV. A bandpass filter $(1-50 \mathrm{~Hz})$ was applied to the signal and analysis performed using custom written MATLAB software. Patients were stratified based on amiodarone therapy. Patients were followed up to 12 months for AF recurrence. 
Results: Four patients were excluded due to lack of follow up and spontaneous reversion to sinus rhythm (SR) just before DCCV. Of the 52 patients analysed, successful DCCV to SR occurred in $98.1 \%$ and maintenance of SR at 12 months in $44.2 \%$. In patients on amidarone $(\mathrm{n}=23)$, PWDc was shorter in those who maintained SR at 12 months vs. those who had remission to $\mathrm{AF}(83.13 \mathrm{~ms}$ vs $112.2 \mathrm{~ms}, \mathrm{P}=0.043)$. ROC analysis exhibited a cut-off of $>86.67 \mathrm{~ms}$ for recurrence with AUC of 0.77 , sensitivity of $78.57 \%$ and specificity of $77.78 \%$ (Figure 1). PWA and TS were not significantly predictive of outcome at 12 months in patients who were not on amiodarone $(n=29)$.

Conclusions: In patients who were on amiodarone at DCCV, increased PWDc measured by BSM immediately after DCCV was associated with AF relapse at 12 months. This may be due to atrial remodelling or substrate related issues. A cut-off value of $>86.67 \mathrm{~ms}$ was predictive of $\mathrm{AF}$ recurrence

\section{AFS 2021-44}

Uploaded File(s)

Body surface mapping of $\mathbf{P}$-waves in sinus rhythm to predict recurrent following cardioversion for atrial fibrillation
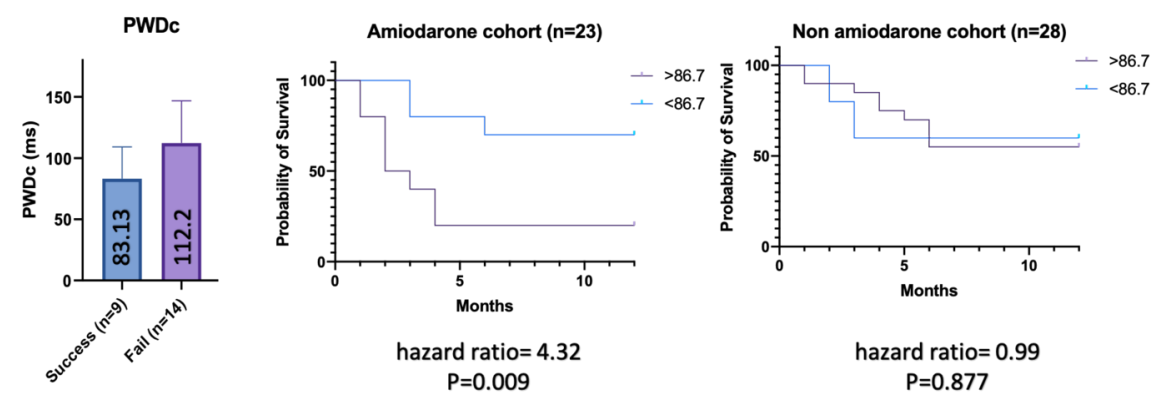

\section{AFS 2021-45}

Abstract Title: A Rare Complication in a Complicated Patient: Pulmonary Vein Stenosis Following Catheter Ablation in a Patient with Corrected Total Anomalous Pulmonary Veins

Author(s): Lukasz Cerbin, MD

\section{Co-Author(s):}

Christopher Barrett, MD - University of Colorado Anschutz Medical Campus

Amneet Sandhu, MD - Veterans Administration Eastern Colorado Health Care System

Alexis Tumolo, MD - University of Colorado Anschutz Medical Campus

Johannes Von Alvensleben, MD - Children's Hospital Colorado

Matthew Zipse, MD - University of Colorado Anschutz Medical Campus

University of Colorado Anschutz Medical Campus

12631 East 17th Avenue, B130 Aurora, CO 80045

Introduction | Objectives: Pulmonary vein stenosis (PVS) is a known complication associated with radiofrequency ablation (RFA) of the pulmonary veins. We present a patient with surgically corrected total anomalous pulmonary venous return (TAPVR) who developed PVS following RFA within an unroofed coronary sinus (CS) for atypical atrial flutter.

Methods: NA 
Results: A 39 year old man with cardiac-type TAPVR, with the confluence of pulmonary veins draining into the CS surgically unroofed into the left atrium in infancy, was referred to our institution for complications related to RFA. He had previously undergone ablation of symptomatic atypical flutter with RFA of the superior left atrium extending into the unroofed CS ostium, to the pulmonary vein confluence. Despite acute success, atrial flutter recurred weeks later prompting initiation of propafenone. In the following months, he noted recurrent respiratory tract infections, dry cough and palpitations resulting in referral for repeat RFA. Pre-procedural CTA demonstrated marked dilation of the left superior and inferior pulmonary veins (LSPV/LIPV) and moderate dilation of the right superior and inferior pulmonary veins (RSPV/RIPV), but no definite PVS. He underwent RFA from the roof of the left atrium into the antrum of the LIPV within the unroofed CS, during which he acutely developed hemoptysis and hypoxemia leading to transfer to our center. CTA demonstrated occlusion of the LIPV, severe stenosis of the LSPV, and moderate stenosis of the right sided pulmonary veins. The patient underwent stenting of the LSPV with staged stenting of right sided veins months later without recurrence of symptoms.

Conclusions: This patient with TAPVR developed severe PVS following RFA within an unroofed CS. Due to the unique anatomy and potential risk for PVS, congenital heart disease patients with a common pulmonary vein confluence may benefit from pulmonary vein isolation via circumferential ablation around the entire confluence rather than RFA near PVs within the unroofed CS, as was done in this case ${ }^{3}$. Multiplanar imaging is critical in preprocedural planning of RFA in this patient population and diagnosing PVS. Pulmonary vein stenting is the definitive treatment.

AFS 2021-45

\title{
Uploaded File(s)
}

Coronal and Axial CT Images of Pulmonary Venous Drainage into the Unroofed Coronary Sinus with Pulmonary Vein Stenosis

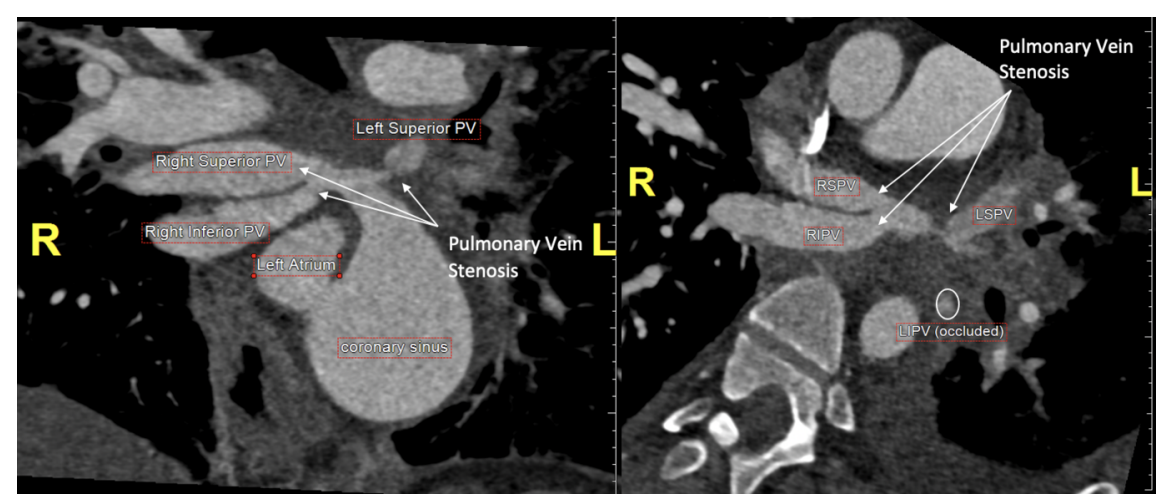

\author{
AFS 2021-46 \\ defect \\ Author(s): Louisa O'Neill, MB Bch BAO \\ Co-Author(s): \\ Iain Sim, MBBS \\ Daniel O'Hare, BMBS \\ John Whitaker, MB BCh MA \\ Rahul Mukherjee, MBBS
}

Abstract Title: AF Substrate Characterisation in Patients with an uncorrected secundum atrial septal 
Caroline Roney, PhD

Orod Razeghi, $\mathrm{PhD}$

Steven Niederer, $\mathrm{PhD}$

Alam Harith, MD

Eric Rosenthal, MD

Matthew Jones, MD

Matthew Wright, MBBS

Alessandra Frigiola, MD

Vivienne Ezzat, MD

Mark O'Neill, MB BCh BAO

Steven Williams, BSc MBChB

King's College London

4th Floor North Wing, St Thomas' Hospital Westminster Bridge Road London SE1 7EH

Introduction | Objectives: Uncorrected atrial septal defects (ASD) are associated with AF but the biatrial arrhythmia substrate is ill-defined in this cohort. We aimed to characterise the extent of electrical and structural remodelling in ASD patients, hypothesising that remodelling and ectopic foci would predominate in the RA and may be related to age and shunt fraction.

Methods: Bi-atrial invasive assessment of voltage, effective refractory periods (ERP) and conduction velocity (CV) and restitution properties was performed during percutaneous ASD closure. Late gadolinium enhancement cardiac MRI (CMR) was performed prior to ASD closure to quantify bi-atrial fibrosis. Ectopic triggers for $\mathrm{AF}$ were assessed with isoprenaline provocation and on Holter monitoring. Comparison was made to non-congenital heart disease AF patients.

Results:Electrical Assessment In 21 ASD and 21 control patients proportion of RA low voltage $(<$ $0.5 \mathrm{mV})$ and scar $(<0.05 \mathrm{mV})$ was greater in ASD than control patients $(\mathrm{P}=0.02$ and $\mathrm{P}=0.039)$. Low voltage and scar areas were greater in the RA than the LA in ASD patients $(\mathrm{P}=0.002$ and $\mathrm{P}=0.010)$. In both atria steeper $\mathrm{ERP}(\mathrm{RA} ; \mathrm{P}=0.005, \mathrm{LA} ; \mathrm{P}=0.016)$ and $\mathrm{CV}$ restitution $(\mathrm{RA} ; \mathrm{P}=<0.001$, $\mathrm{LA} ; \mathrm{P}=0.007)$ was seen in ASD than control patients. CMR Assessment In 36 ASD and 36 control patients bi-atrial fibrosis burden on CMR was significantly greater in ASD than control patients $(\mathrm{P}<0.001)$. RA fibrosis burden was greater in ASD patients with vs without atrial arrhythmias $(\mathrm{P}=0.034)$. Arrhyhmia Triggers During isoprenaline infusion and on non-invasive Holter assessment no differences in atrial ectopy origin was seen in ASD vs control patients. Remodelling and Exposure to Shunt No relationship was seen between the degree of structural or electrical remodelling and patient age or shunt fraction in ASD patients.

Conclusions: Electrical and structural remodelling is present to a greater degree in ASD vs AF control patients and predominates in the RA. RA fibrosis is associated with atrial arrhythmia suggesting a role for CMR in non-invasive risk assessment in these patients. Differences in ERP and CV restitution between ASD and control patient offer mechanistic insight into atrial arrhythmia. Further work is needed to fully define the arrhythmia substrate and guide therapy in this cohort.

\section{AFS 2021-46 \\ Uploaded File(s)}




\title{
Characterisation of atrial electrical and structural remodelling in ASD patients
}

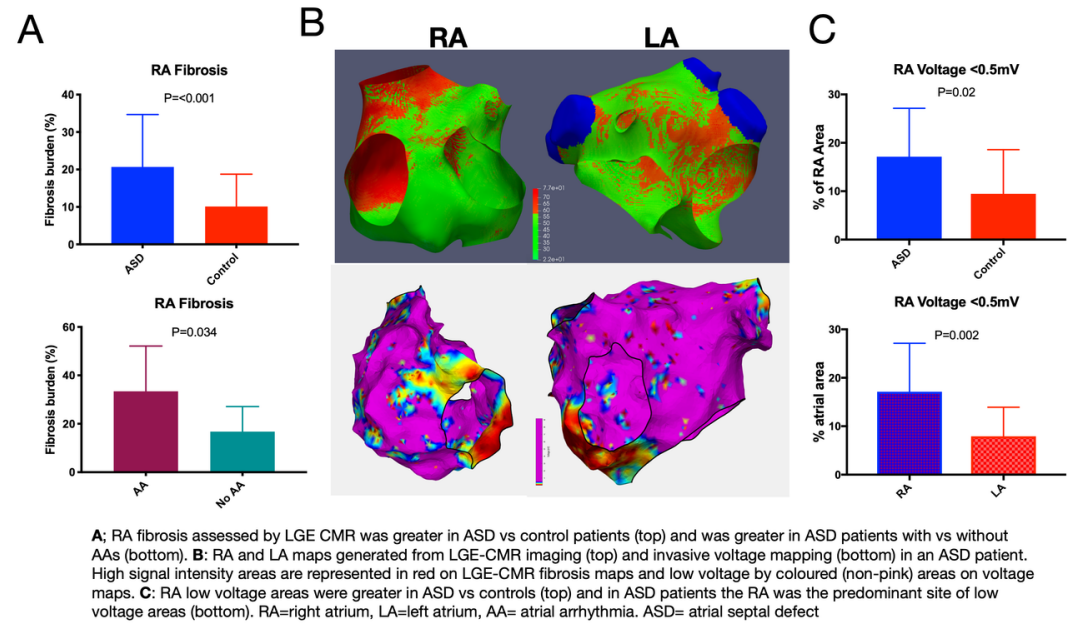

\begin{abstract}
AFS 2021-47
Abstract Title: Minimization of Radiation Exposure in the Modern EP Lab
\end{abstract}

Author(s): Dale Yoo, MD

Co-Author(s):

Emily Buchanan, BS - Research Coordinator, Heart Rhythm Specialists

Texas Health Presbyterian Allen

1105 N Central Expy, Allen, TX 75013

Introduction | Objectives: Radiofrequency ablations (RFA) have been shown to be superior to medical management for reducing recurrence of arrhythmias. Despite current guidelines which detail that the usage of fluoroscopy indicate that the "as low as reasonably achievable", radiation risk for RFA continues to be a moderate complication of ablations, RFAs use and average of 19 minutes or 210 mGy of ionizing radiation which is estimated to increase the risk of a fatal cancer for patients by up to $0.1 \%$ per procedure. Use of highdensity cardiac mapping systems in tandem with intracardiac echocardiography (ICE) has revolutionized the RFA workflow and allowed physicians to perform radiation-free procedures.

Methods: Retrospective Chart Analysis was performed on procedure records detailing ablations performed between January 1, 2013 and December 31, 2019. All patients underwent ablation procedures without the use of fluoroscopy via utilization of three-dimensional mapping systems. A total of 1936 consecutive nonepicardial ablation procedures were analyzed for procedure duration, use of fluoroscopy, and complication rates.

Results: Average procedure time of RFAs $(157 \pm 46$ mins) was comparable to the literature (163 \pm 41 mins). Zero ionizing radiation was used in these cases ( 0 mins, 0 mGy) as compared to traditional RFA procedures (19mins, $210 \mathrm{mGy}$ ). Complications were observed in $1.08 \%$ of cases as compared to tradition RFAs with complication rates of $2.90 \%$. Lead displacement which is a unique complication of the zero-fluoroscopy technique occurred in $0.10 \%$ of all cases. Vascular complications including hematoma, pseudoaneurysm, and $\mathrm{AV}$ fistula were observed in $0.52 \%$ of cases as compared with traditional RFA vascular complications 
at $1.40 \%$. Pericardial effusion and/or cardiac tamponade was observed in $0.52 \%$ of cases as compared with historical values at $1.70 \%$. No deaths were observed as compared to historical rates of $0.06 \%$.

Conclusions: Mitigating radiation exposure is crucial to the field of electrophysiology, since RFA procedures require extensive imaging throughout multiple procedures. In the era of new technologies that allow a physician to effectively and safely perform RFAs without the aid of fluoroscopy, we must actively work to foster a no exposure, no radiation mindset in the medical community.

\section{AFS 2021-48}

Abstract Title: Safety and efficiency of a novel RF balloon catheter for the treatment of atrial fibrillation: a preliminary single Center experience

Author(s): Gaetano Fassini, MD

Co-Author(s):

Stefania Riva, MD

Francesca Pizzamiglio, MD

Maria Antonietta Dessanai, MD

Valentina Ribatti, MD, PhD

Giulia Vettor, MD

Massimo Moltrasio, MD

Claudio Tondo, MD, FESC, FHRS

Centro Cardiologico Monzino IRCCS University of Milan

Via Parea 4 Milano

Introduction | Objectives: Pulmonary vein isolation (PVI) is the cornerstone of catheter-based therapy for patients(Pts) with atrial fibrillation (AF). Single-shot techniques were introduced to improve the procedure. A new multi-electrode radiofrequency balloon catheter (RFB) provides PVI with its 10 irrigated, flexible gold electrodes. The concurrent use of a 10-pole circular diagnostic catheter provides real-time PV recordings. We analyzed time-dependent changes in procedural parameters, acute success, complication rates during our initial experience in subjects with drug refractory, symptomatic, paroxysmal AF.

Methods: 6 patients (age 63.5+-6,8 yrs, 33.3\% male) underwent PVI using the RFB under conscious sedation. Esophageal temperature monitoring and phrenic nerve pacing were performed in all patients. Based on animal Lab and first human experiences, baseline impedance (90-110?) and baseline temperature $(<$ $31 \mathrm{Cdeg}$ ) were set as indicators of optimal electrode-tissue contact. Accordingly, contiguous lesion formation when simultaneously ablating from adjacent electrodes were strongly identified by impedance drop and temperature rise. Intracardiac echocardiography confirmed optimal balloon positioning and stability during RF delivery. Radiofrequency energy was delivered simultaneously from all electrodes, up to 20 s posteriorly and $60 \mathrm{~s}$ anteriorly.

Results: $8.3+-4.82$ applications were delivered per patient; PVI was achieved in $100 \%$ of the 23 targeted PVs. An example of the balloon positioning both on Carto system and on echo is shown in Figure 1. The balloon dwell time was $37.5+-24.5 \mathrm{~min}$. The overall procedure time was $68.3+-32.9 \mathrm{~min}$, and the fluoroscopy time was $12.3+-5.2 \mathrm{~min}$. The first-pass isolation was achieved in $79.6 \%$ of the assessed PVs; with additional RFB applications to the remaining 5 PVs, all PVs were isolated. Mean time to isolation was $17.5+10.6$ sec, obtained in all cases combining the recordings from the decapolar catheter and the balloon electrodes. Acute reconnection was provoked in only 2 PVs. No acute or delayed complications occurred. 
Conclusions: This preliminary experience demonstrated a remarkable acute safety profile and effectiveness of the new RFB.

\section{AFS 2021-48}

\section{Uploaded File(s)}

Fig.1

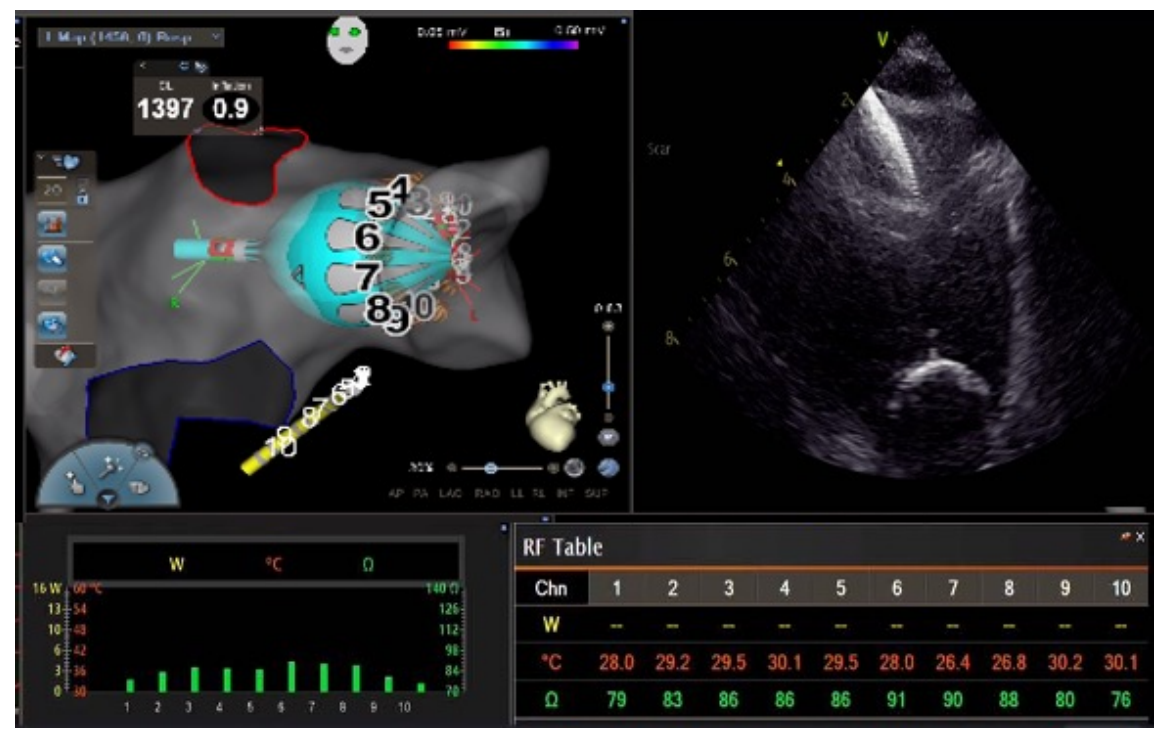

\section{AFS 2021-49}

Abstract Title: Prevalence of Cerebral Small Vessel Diseases in Oral Anticoagulant-Related Intracerebral Hemorrhage

Author(s): Alvin S. Das, MD

Co-Author(s):

Robert Regenhardt, MD, PhD

Elif Gokcal, MD

Mitchell Horn, BSc

Nader Daoud, BA

Joshua Goldstein, MD, PhD

Anderson Christopher, MD

Patel Aman, MD

William Kimberly, MD, PhD

Viswanathan Anand, MD, PhD

lee Schwamm, MD

Jonathan Rosand, MD

Steven Greenberg, MD, PhD 
Mahmut Gurol, MD

Massachusetts General Hospital

55 Fruit Street, Boston, MA 02114

Introduction | Objectives: Limited data exists on the predominant cerebral small vessel disease (CSVD) type underlying oral anticoagulant-related intracerebral hemorrhage (OAC-ICH), including non-vitamin $\mathrm{K}$ antagonist oral anticoagulant-related ICH (NOAC-ICH) and vitamin K antagonist-related intracerebral hemorrhage (VKA-ICH). Herein, we evaluated the two common types of CSVD, hypertensive-CSVD (HTNCSVD) and cerebral amyloid angiopathy (CAA) in atrial fibrillation (AF) patients with NOAC-ICH and VKA-ICH.

Methods: In a prospective database of consecutive non-traumatic ICH patients admitted to a single referral center (April 2003 to December 2019), brain MRI scans were reviewed to diagnose probable CAA (using modified Boston criteria) or HTN-CSVD (the presence of a deep macrobleed with strictly deep or no microbleeds $[\mathrm{MBs}]$ ). The frequency of additional magnetic resonance imaging (MRI) markers of high ICH risk including lacunes and cortical superficial siderosis (cSS) were also assessed.

Results: 1841 patients were included in this study, of which 398 (21.6\%) had AF. In this cohort, 53 experienced a NOAC-ICH and 302 experienced a VKA-ICH. The in-hospital case fatality was $39.6 \%$ for NOAC-ICH and $47.0 \%$ for VKA-ICH, both higher than non-OAC-ICH (35.3\%, $p<0.05$ for both comparisons). The prevalence of HTN-cSVD was similar between NOAC-ICH and VKA-ICH $(28.1 \%$ in NOAC-ICH and $27.8 \%$ in VKA-ICH, $p=0.97$ ). Probable CAA was diagnosed in $28.1 \%$ of NOAC-ICH compared to $24.6 \%$ of VKAICH $(p=0.66)$. The frequency of lacunes, cSS, and MBs was also similar between groups. Moreover, $96.9 \%$ of patients with NOAC-ICH had at least one neuroimaging marker of high ICH risk compared to $86.4 \%$ of patients with $\mathrm{sICH}(p=0.087)$.

Conclusions: CAA represents a substantial underlying pathology in both NOAC-ICH and VKA-ICH. As $97 \%$ of NOAC-ICH patients had high ICH risk markers on MRI: 1) patients without these markers are unlikely to have ICH while on NOACs, 2) nonpharmacological stroke prevention methods can be considered in the presence of these markers.

\section{AFS 2021-50}

Abstract Title: Periprocedural safety and efficacy of left atrial appendage closure with Watchman device versus Watchman-FLX device

Author(s): Andre Briosa e Gala, MD

Co-Author(s):

Michael Pope, BM - EP Research Fellow, Oxford University Hospitals NHS Foundation Trust

Milena Leo, MD - Cardiology Consultant, Oxford University Hospitals NHS Foundation Trust

Timothy Betts, MD - Cardiology Consultant, Oxford University Hospital NHS Foundation Trust

Oxford University Hospital NHS Foundation Trust

Headley way, Oxford, OX39DU, UK

Introduction | Objectives: The Watchman (WM) device has robust periprocedural safety and efficacy (PREVAIL, PROTECT and EWOLUTION registry). The new iteration, Watchman-FLX (WM-FLX), has an improved design enabling implantation in shallower appendages and can be recaptured after full or partial deployment. However, there is no comparative data on its safety and efficacy. This study sought to compare the 30-day periprocedural safety and efficacy in patients undergoing left atrial appendage occlusion (LAAO) device implantation with a WM or a WM-FLX in a large UK tertiary centre. 
Methods: This retrospective study included all patients in our institution from January 2010 to December 2020 planned for implantation of a WD or WD-FLX device. Adverse events at 30-days were identified by reviewing electronic patient records, procedural and imaging reports

Results: A total of 193 consecutive patients ( $74 \pm 8$ years old, $73 \%$ males, $81 \%$ previous major or lifethreatening bleed, $42 \%$ previous ischaemic stroke, $\mathrm{CHA}_{2} \mathrm{DS}_{2}$-VASc score $4.4 \pm 1.2$, HASBLED score $3.2 \pm 0.8$ ) were included, 138 patients in the WM group and 54 in the WM-FLX group. There were no significant differences in baseline demographics. The acute procedural success was similar $(97.8 \% \mathrm{WM}$ and $98.2 \% \mathrm{WM}-$ FLX, $\mathrm{p}=0.8)$ but a shorter fluoroscopy time ( 12.4 vs. 5.82 minutes, $\mathrm{p}=0.01)$, total procedure time ( 56.1 vs. 44.4 minutes, $\mathrm{p}=0.01)$ and length of stay ( 1.4 vs. 0.8 days, $\mathrm{p}<0.01)$ was observed in the WM-FLX group. Complete seal was achieved in $92 \%$ of patients with a WM and $96 \%$ of patients with a WM-FLX $(\mathrm{p}=0.7) ; 1$ patient in the WM group had a moderate leak. The mean number of device redeployments was also similar (0.60 WM vs 0.3 WM-FLX, $\mathrm{p}=0.50$ ). At 30-days, no significant difference was observed in the rate of major and minor procedural complication rate between the WM and WM-FLX groups ( 4.3 vs $1.8 \%$ [ $\mathrm{p}=0.7]$ and $4.3 \%$ vs $0[\mathrm{p}=0.2]$, respectively).

Conclusions: The WM-FLX has similar excellent safety and effectiveness at acutely sealing the LAA as the WM in safety and efficacy and was associated with shorter fluoroscopy and procedure times.

\section{AFS 2021-50}

\section{Uploaded File(s)}

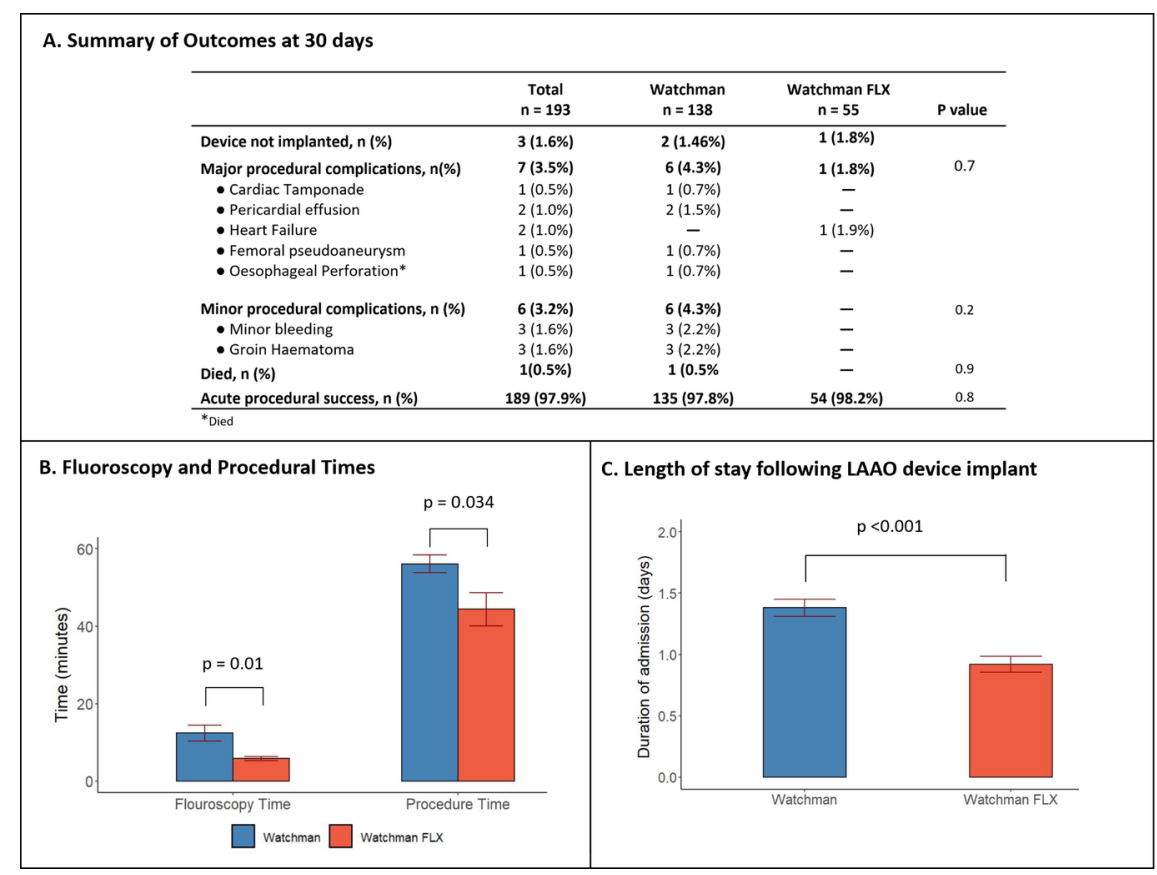

\section{AFS 2021-51}

Abstract Title: Left atrial conduction velocity in paroxysmal and persistent atrial fibrillation Author(s): Daniel O'Hare, BMBS, BEng

\section{Co-Author(s):}

Rokas Bendikas, MEng

Irum Kotadia, MBBS - King's College London 
Iain Sim, MBBS - King's College London

Louisa O'Neill, MB, BCh, BAO - King's College London

Caroline Roney, PhD - King's College London

John Whittaker, MBBCh MA PhD - King's College London

Steven Niederer, PhD - King's College London

Mark O'Neill, MBBCh BAO DPhil - King's College London

Steven Williams, BSc MBChB PhD

King's College London

Westminster Bridge Rd, Bishop's, London SE1 7EH

Introduction | Objectives: Slowing of atrial conduction velocity (CV) shortens the cardiac excitation wavelength and enables a greater number of wave fronts to be sustained within the atria, thereby perpetuating atrial fibrillation (AF). A reduction in atrial $\mathrm{CV}$ is a common finding among patients with $\mathrm{AF}$ when compared to controls. The objective of this study was to determine if differences in conduction velocity can be measured between patients with paroxysmal (PAF) and persistent atrial fibrillation (PsAF).

Methods: A retrospective analysis was performed in 180 patients with atrial fibrillation (90 PAF, 90 PsAF) presenting for index PVI between December 2015 and June 2018. Patients were included if a high-density CARTO map was collected from the left atrium during CS pacing prior to ablation lesions. Conduction velocity was calculated offline using openCARP, an open-source cardiac electrophysiology simulator. For regional analysis, the left atrium was segmented into five areas: Roof, anterior, septal, posterior and lateral. One-year recurrence rates were assessed in 149 of 180 patients (83\%).

Results: The average age of patients was similar in both groups (61.1 vs $61.0 \mathrm{yrs}, \mathrm{p}=0.98)$. Left atrial surface area was significantly larger in the PsAF group (197.4 vs $212.7 \mathrm{~cm} 2, \mathrm{p}=0.002)$. Overall average CV was similar in the PAF and PsAF groups $(0.68 \pm 0.16$ vs $0.69 \pm 0.09 \mathrm{~mm} / \mathrm{ms}, \mathrm{p}=0.70)$ (Fig 1$)$. Increasing atrial size did not significantly correlate to CV slowing $(\mathrm{p}=0.16)$. There was no variation in regional CV in 4 of the 5 left atrial regions. (Anterior, Septal, Lateral and Posterior). There was, however, a significant increase in the conduction velocity in the Roof segment of the PsAF group ( $p=0.04)$ (Fig 2). There was no difference in overall or regional $\mathrm{CV}$ between patients who had a recurrence of atrial arrhythmia within one year, versus the group who remained in sinus rhythm.

Conclusions: This study demonstrates that overall CV is similar in patients with PAF when compared to PsAF at the time of index PVI. The recurrence of atrial arrhythmias within the first year was not significantly correlated to CV.

\section{AFS 2021-51}

Uploaded File(s)

Conduction Velocity Histogram and Left Atrial Maps 


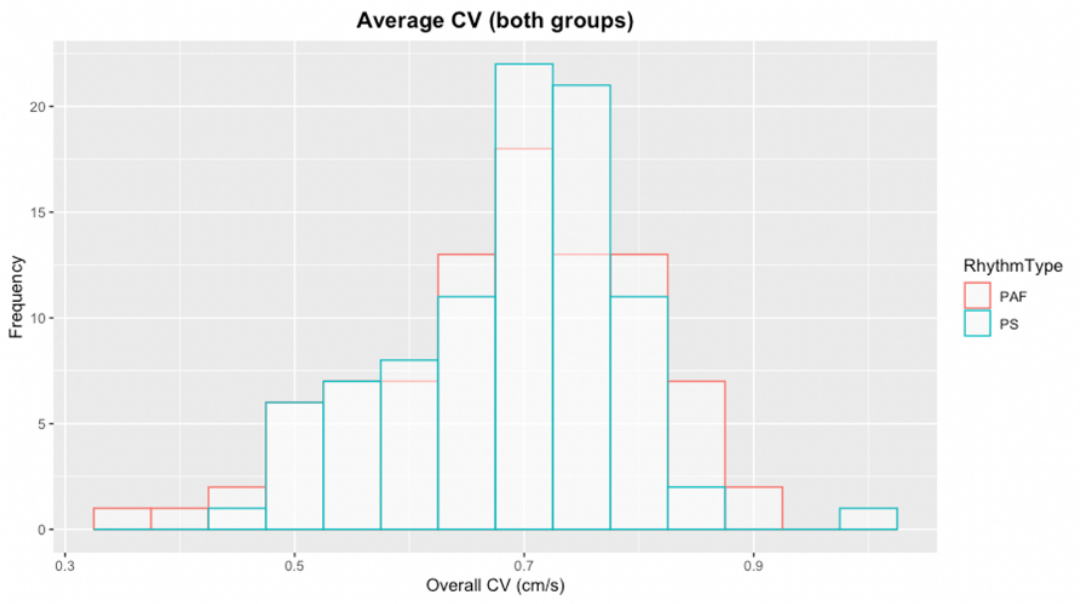

Figure 1: Average CV (mm/ms) of both groups
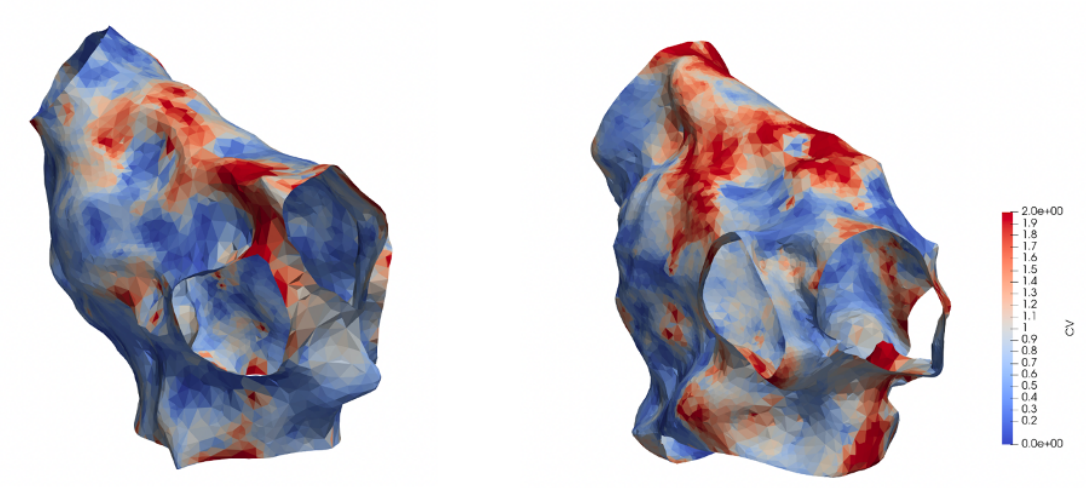

Figure 2: Examples of PsAF left atrial maps with preserved roof conduction

\section{AFS 2021-52}

Abstract Title: All roads lead to Rome but some may be more convoluted - differential behaviour in persistent atrial fibrillation termination during catheter ablation

Author(s): Tiago P. Almeida, BEng, MSc, PhD

Co-Author(s):

Xin Li, BEng, MSc, PhD - Lecturer, University of Leicester

Sidhu Bharat, MBChB, MRCP - Cardiology Research Fellow, University of Leicester

Zakariyya Vali, MBChB, MRCP - Clinical Research Fellow, University of Leicester

Gavin Chu, MB BChir, MA(Cantab), MRCP(UK) - Cardiology Research Fellow, University of Leicester

Peter Stafford, MB BS, MD, FRCP - Consultant Cardiologist, University Hospitals of Leicester NHS Trust

Michela Masè, MSc, PhD - Post-doctoral Fellow, University of Trento

Flavia Ravelli, PhD - University of Trento

Fernando Schlindwein, BEng (1st class Hons), MSc (distinction), CEng, SFHEA, PhD, DSc - Reader, University of Leicester 
G. André Ng, MB ChB (commendation), MRCP (UK), PhD, FRCP, FESC, FHEA, FEHRA - Professor of Cardiac Electrophysiology, University of Leicester

University of Leicester

Glenfield Hospital

Introduction | Objectives: Ablation to treat persistent atrial fibrillation (persAF) remains sub-optimal. Understanding the underlying electrophysiology (EP) of patients that had persAF terminated following ablation is important to identify minimum and optimal set of points for ablation. In the present work, we aimed to understand the EP behaviours of patients who responded differently to additional substrate ablation measured by AF cycle length (AF-CL) changes.

Methods: 11 persAF patients (AF history $57.3 \pm 37.5 \mathrm{mo}$ ) undergoing pulmonary vein isolation followed by substrate ablation guided by NavX (Abbott Laboratories) were identified. All patients had AF terminated to either sinus rhythm or to an organized arrhythmia during substrate ablation. AF-CL was measured in the coronary sinus (10 beats average) before and after each cluster of ablation. The patients were divided in two groups based on AF-CL changes vs. baseline following substrate-guided ablation prior to AF termination: group 1 represents patients with 10\% AF-CL increase or longer (Fig 1A); group 2 represents patients with less than 10\% increase in AF-CL (Fig 1B). 956 bipolar atrial electrograms (AEGs; 5 s, band-pass filtered $30-300 \mathrm{~Hz}, 50 \mathrm{~Hz}$ notch filtered) used to guide ablation with NavX were exported. Attributes were extracted from the AEGs to characterize different EP aspects of each group: NavX's CFE-Mean, CARTO's interval confidence level (ICL), wave similarity index (WSi) and organization index (OI).

Results: 1 patient (patient 4) had AF termination during first cluster ablation. Group 1 (6 patients) showed shorter AF-CL when compared to group 2 (4 patients) (Fig 2A). Group 1 needed less ablation to convert AF compared to group 2 (Fig 2B). Additionally, group 1 showed more fractionated AEGs, with lower CFE-Mean and higher ICL (Fig 3A and 3B), lower WSi and OI (Fig 3C and 3D).

Conclusions: AF-CL changes were more evident in patients with shorter baseline AF-CL. These patients, however, needed less ablation to terminate AF despite seemingly more complex arrhythmia. These counterintuitive results may suggest underlying diversity in mechanisms and highlight the need to tailor ablation according to patient characteristics in order to minimize ablation burden.

\section{AFS 2021-52}

\section{Uploaded File(s)}



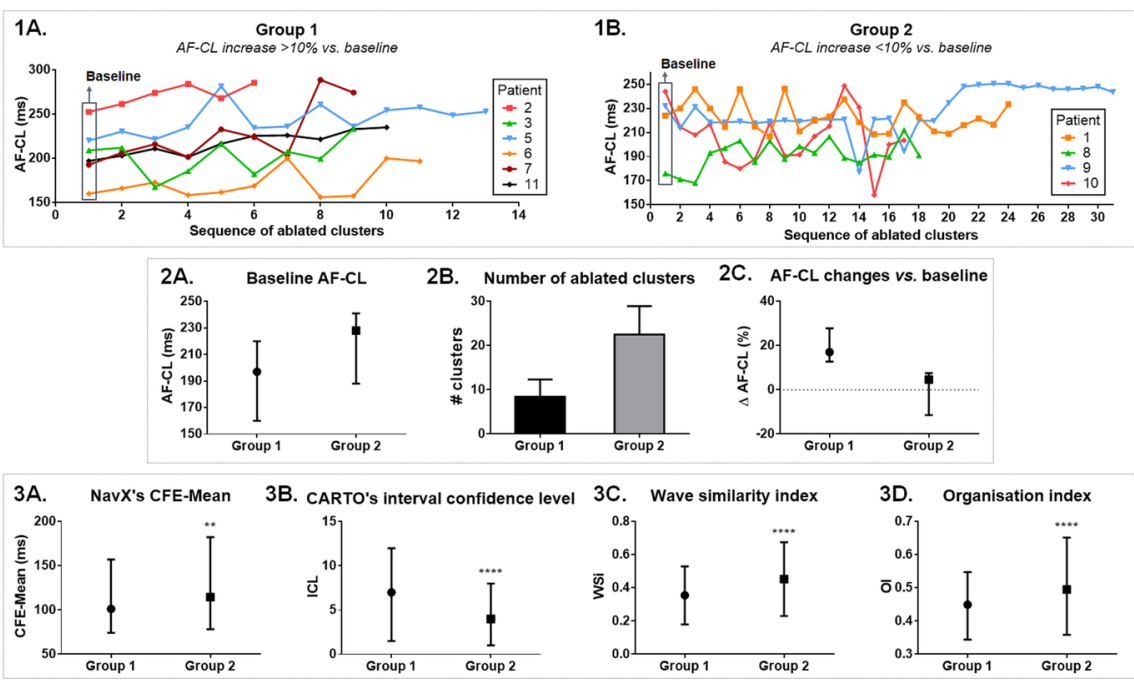

Figure 1. Illustration of the two groups of patients based on AF-CL changes following substrate-guided ablation vs. baseline. Group 1 (A) represents patients with 10\% AF-CL increase or longer vs. baseline. Group 2 (B) represents patients with less than $10 \%$ increase in $\mathrm{AF}-\mathrm{CL}$ vs. baseline. For all patients, $\mathrm{AF}$ terminated in the subsequent ablation point. Figure 2. Characterization of the two groups considering the AF-CL at baseline (A), the amount of ablated clusters up until AF termination (B), and the quantification of AF-CL changes compared to baseline (C) Figure 3. Characterization of the two groups according to the AEG attributes CFE-Mean (A), ICL (B), WSi (C), and Ol (D). ${ }^{* * * *} \mathrm{P} \leq 0.0001 ;{ }^{* *} \mathrm{P} \leq 0.01$

\section{AFS 2021-53}

Abstract Title: Early real-world Experience with Very High Power Short Duration RF Ablation for Atrial Fibrillation

Author(s): Bharat Sidhu, MBChB

\section{Co-Author(s):}

Akash Mavilakandy, MBchB

Ibrahim Antoun, MD

Zakariyya Vali, MBchB

Xin $\mathrm{Li}, \mathrm{PhD}$

Tiago Paggi De Almeida, PhD

Andre Ng, MBChb, PhD

University of Leicester

Department of Cardiovascular Sciences University of Leicester Clinical Sciences Wing Glenfield Hospital Leicester, LE3 9QP

Introduction | Objectives: Pulmonary vein isolation (PVI) for treatment of Atrial fibrillation (AF) using radiofrequency (RF) catheter technology has advanced over the last 10 years with very high power short duration (vHPSD) ablation (90W, $4 \mathrm{~s}$ ) being a new mode of therapy delivery. Having been the first UK centre to adopt this, we hereby report on the procedural data of this early experience compared with standard RF in a contemporary cohort.

Methods: Patients having first time PVI performed under i.v. sedation with vHPSD using QMode+ ( $\mathrm{n}=15$, Qdot, Biosense Webster) were compared with those having standard Ablation Index guided RF 
ablation ( $\mathrm{n}=15$, Thermocool ST/SF, Biosense Webster). Demographics, clinical and procedural data were collected.

Results: Total Ablation time (11 \pm 2 vs. $44 \pm 4$ min, $\mathrm{p}<0.0001)$ and total procedure duration $(152 \pm 7$ vs. $196 \pm 14$ min, $\mathrm{P}=0.02$ ) were significantly shorter in the vHPSD group compared to standard RF whereas fluoroscopy time was similar (Figure 1). Doses of i.v. sedation (midazolam) used was significantly lower in vHPSD than standard RF ( $3.2 \pm 0.8$ vs. $11.1 \pm 1.5 \mathrm{mg}, \mathrm{p}=0.004)$ as was those of i.v. analgesia (morphine) $(11.7 \pm 0.9$ vs. $15.5 \pm 1.2 \mathrm{mg}, \mathrm{P}=0.024)$. When using Qmode $+6.6 \pm 1.4$ additional carina lesions were required compared to $4.8 \pm 1.2$ for standard $\mathrm{RF}(\mathrm{P}=0.338)$. No adverse procedural events were recorded for the vHPSD group while two small non- haemodynamically significant pericardial effusions occurred in the standard RF group, which were conservatively managed.

Conclusions: PVI completed with vHPSD RF ablation (90W, 4s) in paroxysmal AF patients reduced total ablation time and procedure duration with good safety profile. The use of sedation and analgesics was significantly reduced compared with standard RF ablation thus conferring an advantage for procedures to be completed efficiently without general anaesthesia which is particular relevant in resource allocation under concurrent COVID circumstances.

AFS 2021-53

\section{Uploaded File(s)}

Figure 1 Q-Mode Plus vs Standard RF Ablation - Total procedural duration $(\mathrm{p}=0.02)$, total ablation time $(\mathrm{p}<0.0001)$ and fluoroscopy time $(\mathrm{p}=0.1298)$

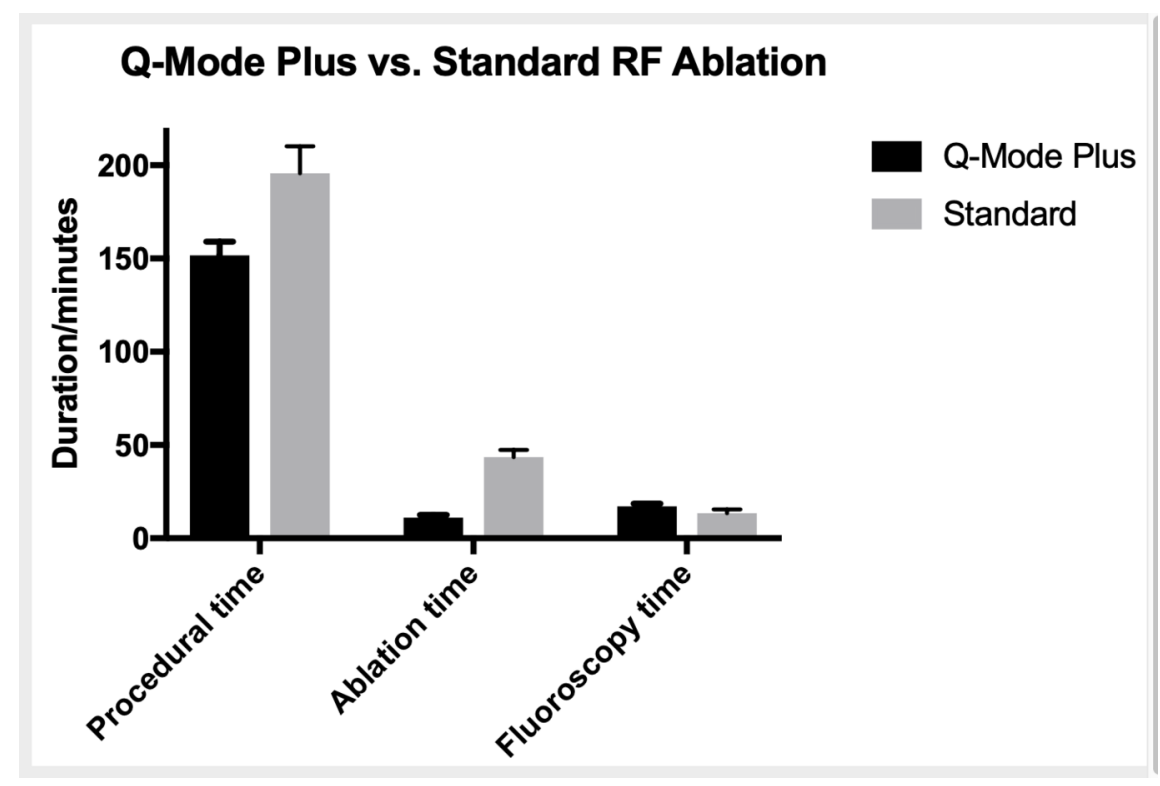

\section{AFS 2021-54}

Abstract Title: Safety and Feasibility of a Conformable Left Atrial Appendage Occlusion Device Author(s): Mohit K. Turagam, MD

Co-Author(s):

Petr Neuzil, MD

Srinivas Dukkipati, MD 
Vivek Reddy, MD

Mount Sinai Hospital

1, Gustav L. Levy Street, New York, NY-10029

Introduction | Objectives: Transcatheter left atrial appendage closure (LAAC) has evolved as an important stroke prevention strategy in AF patients. Limitations of current LAAC devices include the need for precise coaxial delivery into the LAA, potential for traumatic implantation, incomplete LAA seal, and device-related thrombus (DRT). We report, the feasibility and safety of ICE-guided Conformal Left Atrial Appendage Closure Device (Conformal Medical, Inc ) implantation.

Methods: The Conformal Left Atrial Appendage Closure implant is a self-expanding occluder consisting of a cylindrical nitinol endoskeleton (with low-profile anchor barbs around the midpoint) covered with a porous foam cup. In a prospective single center series, under conscious sedation, the Conformal device was delivered under fluoroscopic and ICE guidance. (Figure) After positioning, TEE was placed to confirm ICE findings prior to device release. After closure, dual antiplatelet therapy was given for 6 months.

Results: Fifteen patients underwent LAAC (age 71.3 \pm 10.8 yrs, $33 \%$ male, CHA2DS2-VASc $4.1 \pm 1.7$, LAA diameter $21.7 \pm 4.5$ ). Implantation was $100 \%$ successful, taking $55.1 \pm 20.6$ min, using $41.5 \pm 14.5$ cc contrast. TEE evaluation prior to final release revealed adequate seal in all patients. There were no procedure/devicerelated complications. In 11 patients (4 missed TEE due to COVID-19), TEE at 45-days revealed adequate seal (9 pts-no leak; 1pt-1mm; 1pt-2mm) and no DRT. In 9 patients (6 missed TEE due to COVID-19), TEE at 6 months revealed adequate seal ( 8 patients-no leak; 1patient-1mm) and 1 patient had DRT. Death occurred in 1 patient which was unrelated to the procedure.

Conclusions: The Conformal Left Atrial Appendage Closure Device can be safely implanted with intraprocedural ICE imaging instead of TEE

AFS 2021-54

Uploaded File(s)

ICE-guided implantation of the Conformal Left Atrial Appendage Closure Device

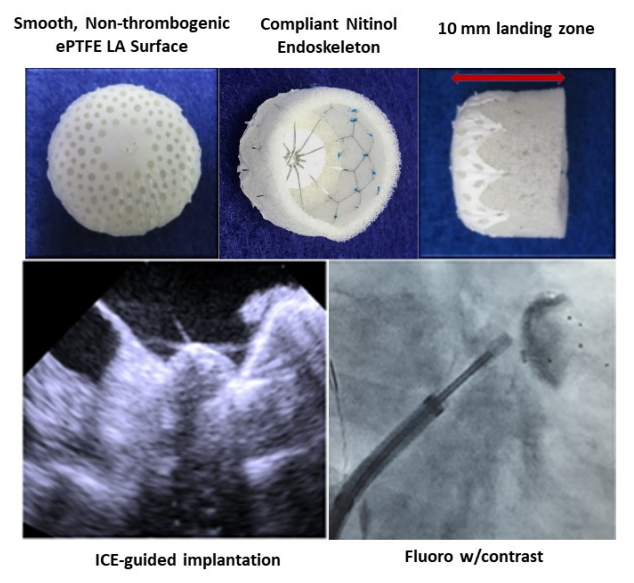

AFS2021-55

Abstract Title: Pulsed Field Ablation Using a Multielectrode Pentaspline Catheter: Clinical Outcomes with an Optimized Waveform 
Author(s): Vivek Reddy, MD

Co-Author(s):

Pierre Jais, MD

Ante Anic, MD

Jan Petru, MD

Moritoshi Funasako, MD

Kentaro Minami, MD

Toni Breskovic, MD

Ivan Sikiric, MD

Lucie Sediva, MD

Milan Chovanec, MD

Petr Neuzil, MD PhD

Icahn School of Medicine at Mount Sinai

One Gustave L Levy Place Box 1030

Introduction | Objectives: Pulsed field ablation (PFA) is a non-thermal energy source notable for its relative preferential effect on myocardial tissue, and not surrounding tissues such as esophagus or phrenic nerve. However, PFA's efficacy/tissue selectivity is dependent on many factors - including waveform characteristics. Thus, outcomes using one set of parameters may not predict outcomes using different parameters. A multielectrode pentaspline PFA system was optimized with prospective remapping studies. Herein, we report the clinical outcomes - durability and recurrent arrhythmias - using this optimized PFA waveform for treating paroxysmal atrial fibrillation (PAF).

Methods: PAF pts underwent pulmonary vein isolation (PVI) with the pentaspline catheter (Farawave; Farapulse Inc) and PFA generator using an optimized PFA waveform (Optiwave; Farapulse Inc). By protocol, invasive remapping was performed at 3 mo; one-year follow-up included weekly TTM and 6-/12mo $24 \mathrm{~h}$ Holters.

Results: At 3 sites, 5 operators performed PVI in 49 pts ( $57 \pm 10$ y, $65 \%$ male, $40 \pm 5 \mathrm{~mm}$ LA diameter), with procedure duration $97.2 \pm 29.1 \mathrm{~min}$ (included 19.0 \pm 13.5 min of protocol-mandated mapping). All PVs (195 of $195 \mathrm{PVs}$ ) were isolated, typically with the first $\sim 3 \mathrm{~s}$ application. In all, there were $8.7 \pm 1.5$ deliveries/PV. Primary safety events included 2 vascular complications (one arising from a non-study device during remap); there was no stroke, TIA, PV stenosis or phrenic nerve injury. Additional safety assessments included screening EGD (11 pts) and screening DW-MRI (5 pts) - all negative for esophagus and brain injury, respectively. Remapping was performed in 44 pts (90\%), revealing durability in 166 of 173 (96\%) PVs. At $337 \pm 63$ days follow-up, with 29 pts reaching study exit at 1 year, freedom from AF or AF/AT/AFL were $85 \pm 6 \%$ and $85 \pm 5 \%$, respectively.

Conclusions: Using an optimized waveform, the pentaspline PFA catheter achieved high PVI durability, which in turn translated to excellent 1-year freedom from recurrent atrial arrhythmia. This information is of particular relevance, as this will be the available PFA waveform for both a forthcoming randomized FDA clinical trial (ADVENT ), and for a commercial system (expected in Europe in Q1-2021).

AFS2021-55 Uploaded File(s) One Year Outcomes Using an Optimized PFA Waveform 

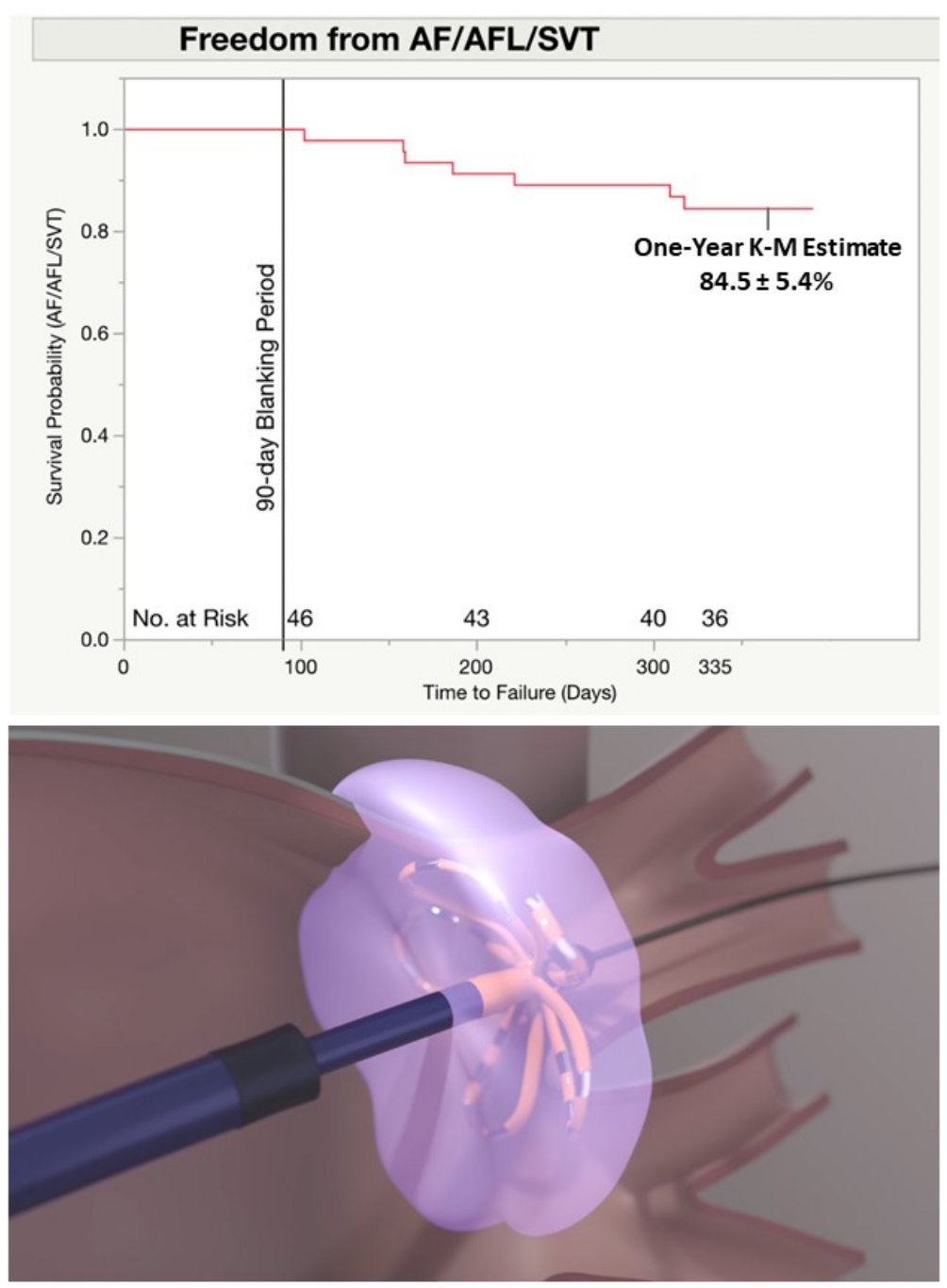

\section{AFS2021-56}

Abstract Title: Dielectrophoretic Red Blood Cell Fusion by Pulsed Electric Fields: Ex vivo and Porcine in vivo Experiments

Author(s): Vivek Reddy, MD

Co-Author(s):

Raj Viswanathan, $\mathrm{PhD}$

Pierre Jais, MD

Icahn School of Medicine at Mount Sinai

One Gustave L Levy Place Box 1030 
Introduction | Objectives: Pulsed field ablation (PFA) has favorable theoretical and empirically-observed safety benefits for treating atrial fibrillation. However, it is important to fully understand the unique biophysics of this novel energy source. Indeed, in vitro studies have shown that electric fields induce dielectrophoretic attraction and, at locally high field strengths, can cause deformation and cellular aggregation by $\mathrm{RBC}$ cell membrane electrofusion. Using a multielectrode PFA catheter, we investigated this phenomenon both ex vivo and in vivo .

Methods: An ex vivo model of PFA-induced RBC electrofusion included a beaker with 400-500 mL of defibrinated blood (Lampire Biological Products, PA) stirred magnetically at $30^{\circ} \mathrm{C}$. Using a pentaspline PFA catheter and custom generator (Farapulse Inc), PFA waveforms were delivered up to $2.0 \mathrm{kV}$. After multiple runs of 30 PFA deliveries each, any electrode-adherent material was subjectively ranked for extent of deposits by 3 - 5 blinded observers: rank of 1 being least and 15 being most. A subset of these tests was repeated in vivo inporcine left atria. Any identified aggregates were sent for histological analysis.

Results: Ex vivo , PFA (using electrical currents $20 \%$ higher than used clinically) resulted in trace deposits of adhered, dark-colored material on some distal electrode edges (Figure ). Consistent with prior reports of RBC electrofusion, histological analysis revealed fused erythrocytes with a small amount of fibrin. Subjective ranking of deposits was improved after implementing refinements to PFA system architecture and catheter construction $(4.3 \pm 3.0)$, vs before $(8.8 \pm 3.8)$. Similar results were observed in vivo : ranks of $5.0 \pm 3.9$ after implementing refinements, vs before $(10.7 \pm 3.0)$.

Conclusions: In ex vivo and in vivo studies, PFA could induce dielectrophoretic attraction of RBCs and, at high local field strengths (not currently being used clinically), can result in electrofusion of cell membranes into macroscopic aggregations. The propensity for RBC electrofusion is sensitive to alterations in device and waveform parameters. While the clinical significance of electrofusion is unknown, it would be prudent for PFA systems to be "tuned" to minimize this phenomenon.

\section{AFS2021-56}

\section{Uploaded File(s)}

Small RBC deposits on distal electrode edges with suboptimal system parameters (left) and eliminated with optimized system parameters (right), and Histopathology slide (below) showing material being composed of fused erythrocytes (FE) and a small amount of fibrin (F). HE stain, 200X magnification. 


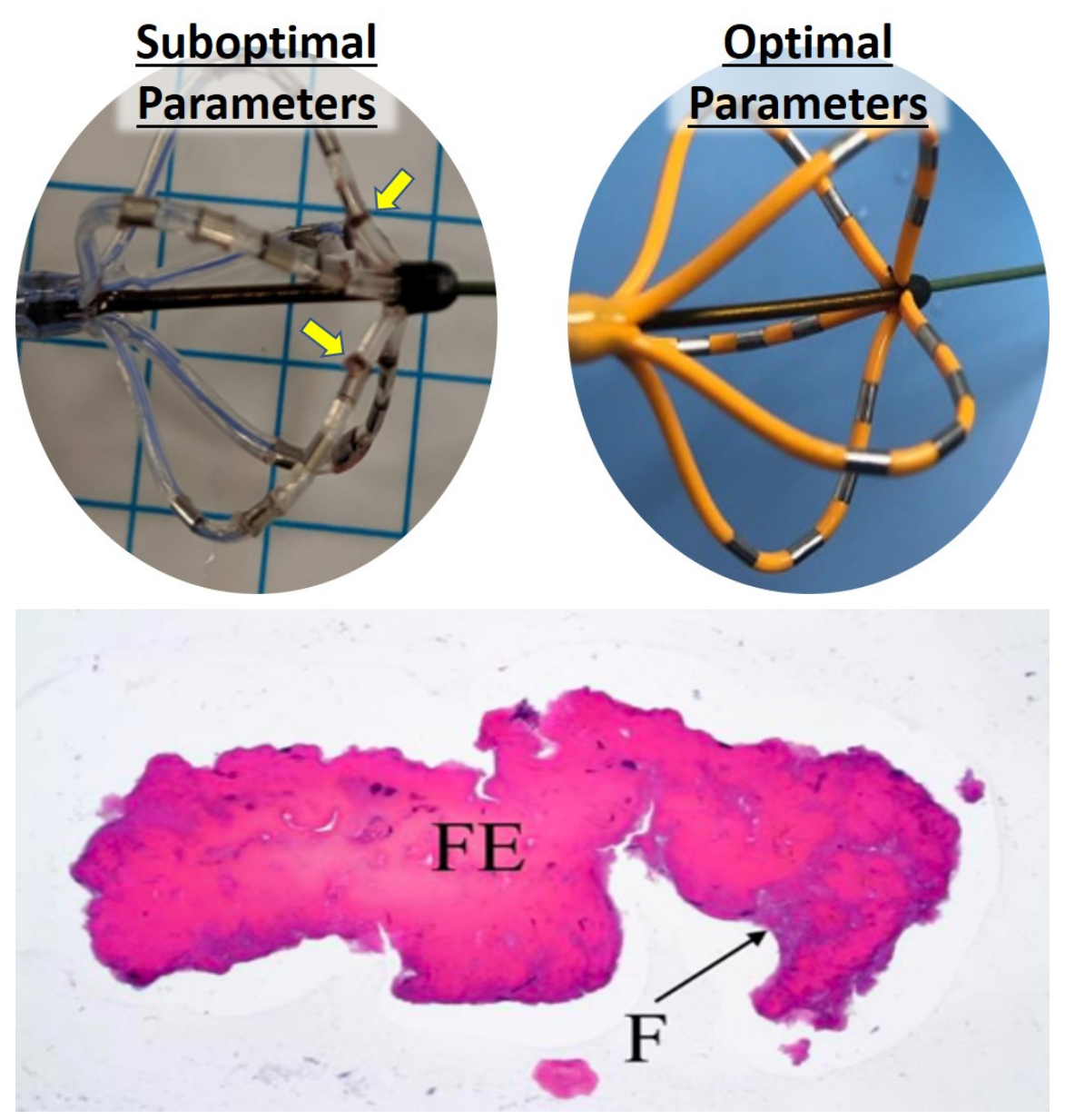

